\title{
Anion Directed Synthesis of a Paddalane and Trisilver Tweezer Complex Based Upon Silver Coordination Chemistry
}

\author{
Paul D. Custer, Jered C. Garrison, Claire A. Tessier and Wiley J. Youngs \\ Department of Chemistry, The University of Akron, Akron, Ohio 44325, USA.
}

\section{Supporting Information}

\section{Table of Contents}

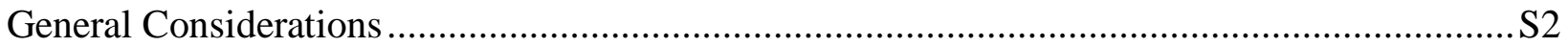

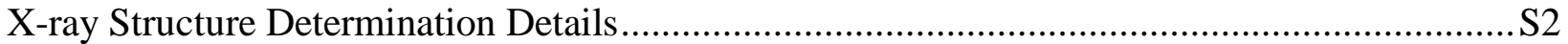

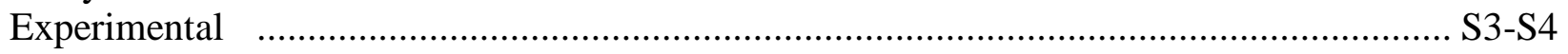

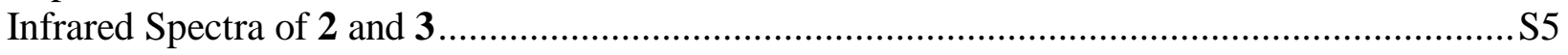

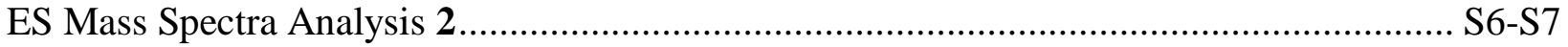

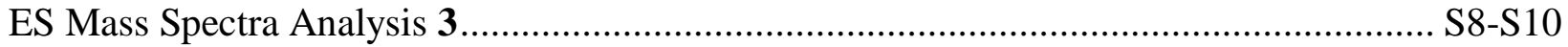

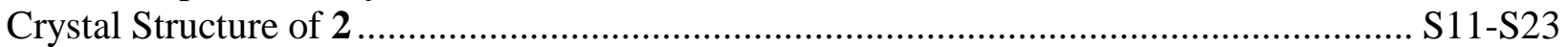

Crystal Structure of 3 .................................................................................... S24-S50

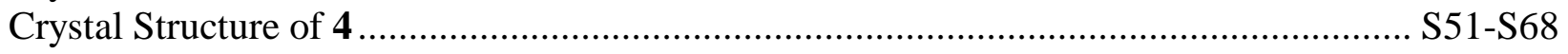




\section{General Considerations}

All manipulations dealing with lithium reagents were carried out under an $\mathrm{N}_{2}$ atmosphere. Phenylacetylene, $\mathrm{AgBF}_{4}$ and $\mathrm{Ag}\left(\mathrm{O}_{3} \mathrm{SCF}_{3}\right)$ were purchased from Aldrich and used without further purification. 1,4,7-trimethyl-1,4,7-triazacyclononane $\left({ }^{\mathrm{Me} 3} \operatorname{tacn}\right)$ was synthesized according to literature procedures. ${ }^{1-3}$ Dry THF was obtained from a PureSolv ${ }^{\mathrm{TM}}$ solvent purification system. ${ }^{1} \mathrm{H}$ and ${ }^{13} \mathrm{C}$ NMR data were recorded on Varian's Gemini $300 \mathrm{MHz}$ and Inova $400 \mathrm{MHz}$ instruments. The spectra were referenced to the residual proton and ${ }^{13} \mathrm{C}$ signals of the deuterated solvents. ${ }^{19} \mathrm{~F}$ NMR data was recorded on a Varian Gemini $300 \mathrm{MHz}$ instrument and referenced to trifluoracetic acid. Mass spectrometry data was collected on a Bruker Daltons (Billerica, MA) Esquire-LC mass spectrometer equipped with ESI. Infrared Spectra were collected as $\mathrm{KBr}$ films using a Bomem B-100 Infrared spectrometer.

\section{X-ray Structure Determination Details}

Crystals of 2, 3 and $\mathbf{4}$ were coated in paratone oil and mounted on a CryoLoop ${ }^{\mathrm{TM}}$ and placed on the goniometer head under a stream of nitrogen cooled to $100 \mathrm{~K}$. The data was collected on a Bruker APEX CCD diffractometer with graphite-monochromated Mo $\mathrm{K}_{\alpha}$ radiation $(\lambda=0.71073 \AA)$. The unit cell was determined by using reflections from three different orientations. The data was integrated using SAINT. ${ }^{4}$ An empirical absorption correction and other corrections were applied to the data using multi-scan SADABS. ${ }^{4}$ Structure solution, refinement, and modeling were accomplished by using the Bruker SHELXTL package. ${ }^{4,5}$ The structure was determined by full-matrix least-squares refinement of $F^{2}$ and the selection of the appropriate atoms from the generated difference map. Hydrogen atom positions were calculated and $\mathrm{U}_{\text {iso }}(\mathrm{H})$ values were fixed according to a riding model.

\footnotetext{
${ }^{1}$ McAuley, A.; Norman, P. R.; Olubuyide, O. Inorg. Chem. 1984, 1938-1943.

${ }^{2}$ Hay, R. W.; Norman, P. R. J. Chem. Soc., Dalton 1979 1441-1445.

${ }^{3}$ Wang, L.; Wang, C.; Bau, R.; Flood, T. C. Organometallics 1996, 15, 491-498.

${ }^{4}$ Bruker (1997). SMART (Version 5.625), SAINT (Version 6.22) and SHELXTL (Version 6.10)

${ }^{5}$ Sheldrick, G. M. (1997). SHELX-97. University of Göttingen, Germany
} 
${ }^{M e}{ }_{\text {tacnRh}}(\mathbf{C C P h})_{3}$ (2). A $500 \mathrm{~mL}$ schlenk flask was charged with a stir bar, $1.978 \mathrm{~g}$ of ${ }^{\mathrm{Me}}$ tacnRhCl $_{3}(5.20 \mathrm{mmol})$ and $100 \mathrm{~mL}$ of dry THF. A separate $100 \mathrm{~mL}$ schlenk flask was charged with $2.28 \mathrm{~mL}$ of phenylacetylene $(20.75 \mathrm{mmol})$ and $50 \mathrm{~mL}$ dry THF. The flask containing the phenylacetylene was cooled to $-78^{\circ} \mathrm{C}, 13.0 \mathrm{~mL}$ of $2.0 \mathrm{M} \mathrm{BuLi}$ was added and stirred for $30 \mathrm{~min}$. The mixture was transferred via canula to the flask, cooled to $-78{ }^{\circ} \mathrm{C}$, containing the ${ }^{\mathrm{Me} 3} \mathrm{tacnRhCl}_{3}$. The reaction mixture was allowed to stir at RT for three days. The flask was opened to air and a solution of wet THF was added drop wise to quench any remaining lithium reagents. All volatiles were removed under vacuum. $\mathrm{CH}_{2} \mathrm{Cl}_{2}(150 \mathrm{~mL})$ was added to the solid and the mixture was stirred for $30 \mathrm{~min}$. The mixture was filtered to remove the $\mathrm{LiCl}$. Evaporation of solvent yielded a brown solid which was washed with hexanes and dissolved in $150 \mathrm{~mL}$ of $\mathrm{CH}_{2} \mathrm{Cl}_{2}$. The solution was extracted with $5 \times 200 \mathrm{~mL}$ of water. The organic phase was dried using $\mathrm{MgSO}_{4}$ and filtered. The solvent was removed under vacuum to afford $\mathbf{2}$ as a light brown solid. Purification of the compound was achieved by dissolving 2 into a minimal amount of $\mathrm{CH}_{2} \mathrm{Cl}_{2}(\sim 50 \mathrm{~mL})$ and the addition of $250 \mathrm{~mL}$ of cold diethyl ether. The resulting white precipitate, 2, was filtered. Yield: $1.958 \mathrm{~g}(3.39 \mathrm{mmol}), 65 \%$; $\mathrm{Mp}>250{ }^{\circ} \mathrm{C} ;{ }^{1} \mathrm{H} \mathrm{NMR}(300$ $\mathrm{MHz}, \mathrm{d}_{6}$-DMSO) $\delta$ 2.92-3.28 (m, 12H, $\mathrm{CH}_{2}$ ), 3.05 (s, 9H, $\left.\mathrm{CH}_{3}\right), 7.02$ - 7.26 (m, 15H, aromatic); ${ }^{13} \mathrm{C} \mathrm{NMR}\left(75 \mathrm{MHz}, \mathrm{d}_{6}\right.$-DMSO) $\delta=51.26\left(\mathrm{~s}, \mathrm{CH}_{3}\right), 58.61\left(\mathrm{~s}, \mathrm{CH}_{2}\right), 98.52\left(\mathrm{~d}, \mathrm{C}_{\beta}, J=9.4 \mathrm{~Hz}\right)$, $111.03\left(\mathrm{~d}, \mathrm{C}_{\alpha}, J=48.5 \mathrm{~Hz}\right), 124.28,127.98,129.27,130.63$ (aromatic carbons). IR (KBr): $v(\mathrm{CC}) 2103(\mathrm{~s}) \mathrm{cm}^{-1}$; ES-MS (8:2-DMSO:CH$\left.{ }_{3} \mathrm{CN}\right)$ cacld for $[\mathrm{M}] \mathrm{Na}^{+}: \mathrm{m} / \mathrm{z} 600.2$, found 600.3; Anal. Calcd for $\mathrm{C}_{33} \mathrm{H}_{36} \mathrm{~N}_{3} \mathrm{Rh}$ : C, 68.63; H, 6.28; N, 7.28. Found: C, 68.62; H, 6.13; N, 7.22.

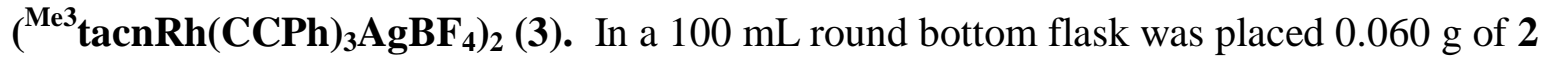
$(0.104 \mathrm{mmol})$ and $60 \mathrm{~mL}$ of $\mathrm{CH}_{3} \mathrm{CN}$. $\mathrm{AgBF}_{4}(0.146 \mathrm{~g}, 0.750 \mathrm{mmol})$ was added to the solution of 2 and stirred for $1 \mathrm{~min}$. All volatiles were removed under reduced pressure. The resulting brown solid was washed with water and filtered. The solid was dissolved in $6 \mathrm{~mL}$ of $\mathrm{CH}_{3} \mathrm{CN}$ and filtered. The resulting solution was allowed to slowly evaporate, in the dark, to give $0.0803 \mathrm{~g}$ of $\mathbf{3}$ as brown crystals. Analysis of $\mathbf{3}$ by x-ray diffraction studies reveals a non-coordinating acetonitrile molecule in the asymmetric unit. The weight of the acetonitrile was taken into account when calculating the yield. Yield: $0.078 \mathrm{~g}(0.101 \mathrm{mmol}), 97 \% ; \mathrm{Mp}>220^{\circ} \mathrm{C}$; ${ }^{1} \mathrm{H}$ NMR (300 MHz, d 6 -DMSO) $\delta 3.10\left(\mathrm{~s}, 18 \mathrm{H}, \mathrm{CH}_{3}\right), 3.29\left(\mathrm{~s}, 24 \mathrm{H}, \mathrm{CH}_{2}\right), 7.06(\mathrm{t}, 12 \mathrm{H}, J=6.9$ $\mathrm{Hz}), 7.14(\mathrm{t}, 6 \mathrm{H}, J=6.9 \mathrm{~Hz}), 7.32(\mathrm{~d}, 12 \mathrm{H}, J=6.9 \mathrm{~Hz}) ;{ }^{13} \mathrm{C}$ NMR $\left(75 \mathrm{MHz}, \mathrm{d}_{6}\right.$-DMSO) $\delta 52.04$ $\left(\mathrm{s}, \mathrm{CH}_{3}\right), 59.87\left(\mathrm{~s}, \mathrm{CH}_{2}\right), 94.12\left(\mathrm{~d}, \mathrm{C}_{\alpha}, J=44.6 \mathrm{~Hz}\right), 103.95\left(\mathrm{~m}, \mathrm{C}_{\beta}\right), 124.10,127.32,127.94$, 131.16 (aromatic carbons); IR (KBr): $v(\mathrm{CC}) 2047 \mathrm{~cm}^{-1}$; ES-MS (acetone) cacld for $\left[\mathrm{M}+\mathrm{BF}_{4}\right]^{+}$and $[\mathrm{M}]^{2+}: \mathrm{m} / z$ 1457.2 $\left[\mathrm{M}+\mathrm{BF}_{4}\right]^{+}$and $685.1[\mathrm{M}]^{2+}$, found 1457.2 and 686.1 ; Anal. Calcd for $\mathrm{C}_{33} \mathrm{H}_{36} \mathrm{~N}_{3} \mathrm{RhAgBF}_{4}$ : C, 51.33; H, 4.70; N, 5.44. Found: C, 50.57; H, 4.56; N, 5.63. 
${ }^{M e 3}{ }_{\operatorname{tacnRh}}(\mathbf{C C P h})_{3}\left(\mathbf{A g}\left(\mathbf{O}_{3} \mathbf{S C F}_{3}\right)\right)_{3} \mathbf{C H}_{3} \mathbf{C N}(\mathbf{4})$. A $100 \mathrm{~mL}$ schlenk flask was charged with $0.176 \mathrm{~g}$ of $2(0.304 \mathrm{mmol})$ and a stir bar. $\mathrm{Ag}\left(\mathrm{O}_{3} \mathrm{SCF}_{3}\right)(0.318 \mathrm{~g}, 1.24 \mathrm{mmol})$ was added, under a nitrogen atmosphere, with the reaction flask shielded from light. The flask was opened to the atmosphere and $25 \mathrm{~mL}$ of acetonitrile was added to the flask. The solution was allowed to stir at $\mathrm{RT}$ for $2 \mathrm{~h}$. The solution was concentrated to $\sim 2 \mathrm{~mL}$. The solution was allowed to slowly evaporate, in the dark, to yield brown crystals. The crystals were rinsed with cold water to remove impurities. Yield $0.373 \mathrm{~g}(0.268 \mathrm{mmol}), 88 \%$; ${ }^{1} \mathrm{H}$ NMR $\left(300 \mathrm{MHz}, \mathrm{d}_{6}\right.$-DMSO) $\delta 3.10$ $\left(\mathrm{s}, 9 \mathrm{H}, \mathrm{CH}_{3}\right), 3.29\left(\mathrm{~s}, 12 \mathrm{H}, \mathrm{CH}_{2}\right), 7.06(\mathrm{t}, 6 \mathrm{H}, J=7.2 \mathrm{~Hz}), 7.14(\mathrm{t}, 3 \mathrm{H}, J=6.9 \mathrm{~Hz}), 7.32(\mathrm{~d}, 6 \mathrm{H}, J$ $=7.2 \mathrm{~Hz}) ;{ }^{13} \mathrm{C} \mathrm{NMR}\left(100 \mathrm{MHz}, \mathrm{d}_{6}-\mathrm{DMSO}\right) \delta 52.00\left(\mathrm{~s}, \mathrm{CH}_{3}\right), 59.83\left(\mathrm{~s}, \mathrm{CH}_{2}\right), 94.04\left(\mathrm{~d}, \mathrm{C}_{\alpha}, J=\right.$ $44.4 \mathrm{~Hz}), 103.93\left(\mathrm{~m}, \mathrm{C}_{\beta}\right), 124.05,127.26,127.87,131.10$ (aromatic carbons); ${ }^{19} \mathrm{~F}$ NMR (300 $\left.\mathrm{MHz}, \mathrm{DMSO}-\mathrm{d}_{6}\right) \delta 0.52\left(\mathrm{~s}, \mathrm{CF}_{3}\right)$. 


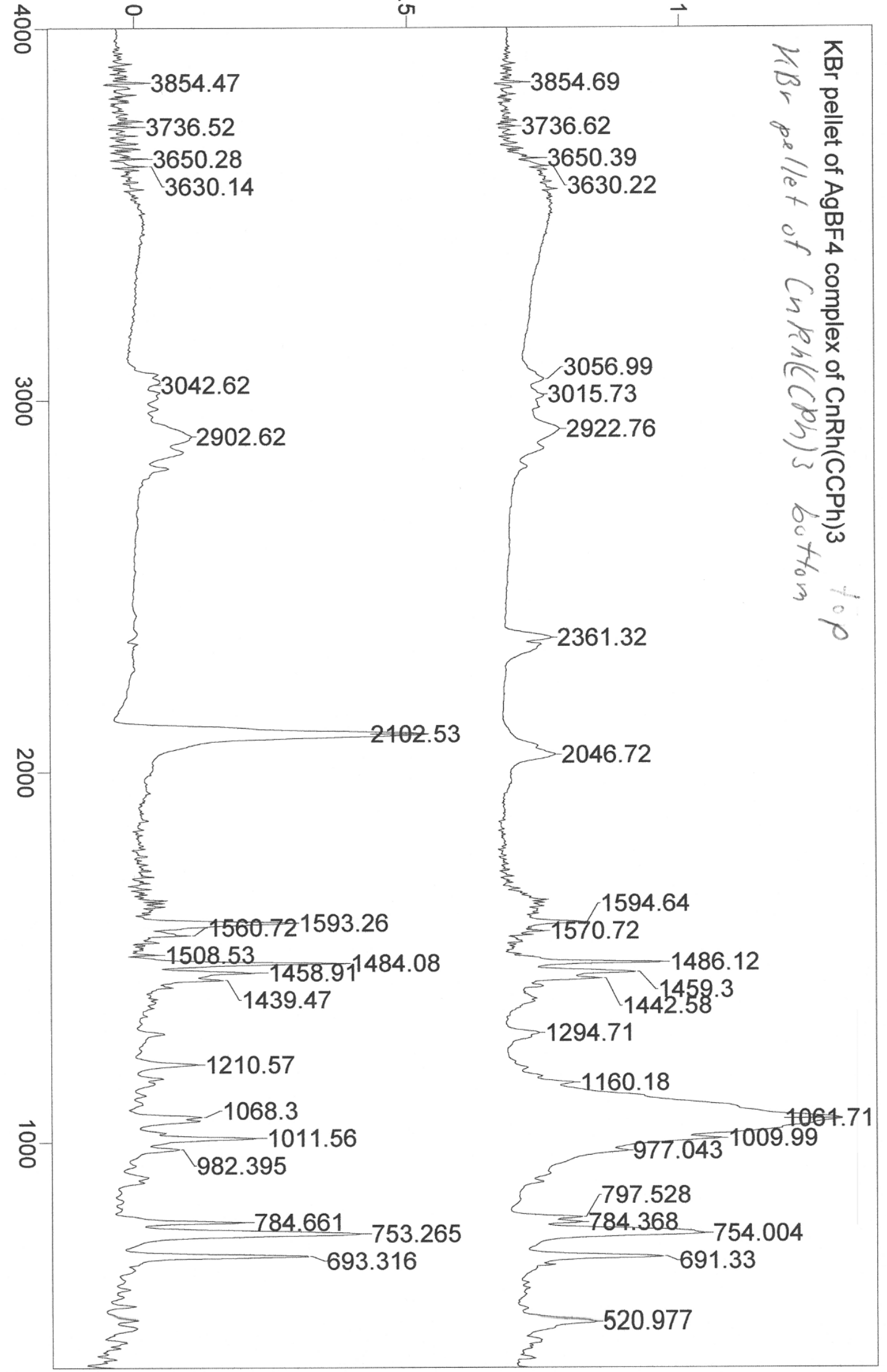

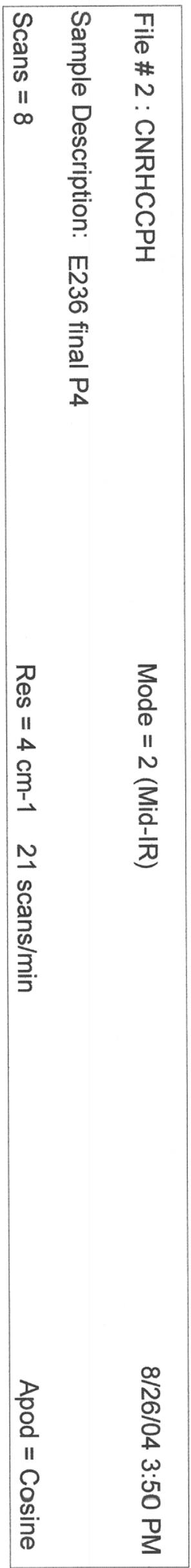




\section{Display Report}

\section{Analysis Info}

Analysis Name

Method

Sample Name

Comment
ES4042 0.d

DEFAULT.MS

research

Youngs/Paul Custer Paul 1 C33 H36 M3 Rh1 $120 / / 5 / 8 / 150 / / 600 / 20 / 100$
Acquisition Date

Operator

02/18/04 16:07:44

nstrument Esquire-LC_00143

577 10-4M DMSO/CH3CN 8:2

\section{Acquisition Parameter}

Ion Source Type

Mass Range Mode

Capillary Exit

Accumulation Time

\section{ESI}

Std/Normal

87.4 Volt

$67227 \mu \mathrm{s}$

$\begin{array}{ll}\text { Ion Polarity } & \text { Positive } \\ \text { Scan Begin } & 150.00 \mathrm{~m} / \mathrm{z} \\ \text { Skim 1 } & 19.4 \text { Volt } \\ \text { Averages } & 40 \text { Spectra }\end{array}$

Averages
Alternating Ion Polarity

Scan End

Trap Drive

Auto MS/MS $\mathrm{n} / \mathrm{a}$

$1000.00 \mathrm{~m} / \mathrm{z}$

49.0

Off

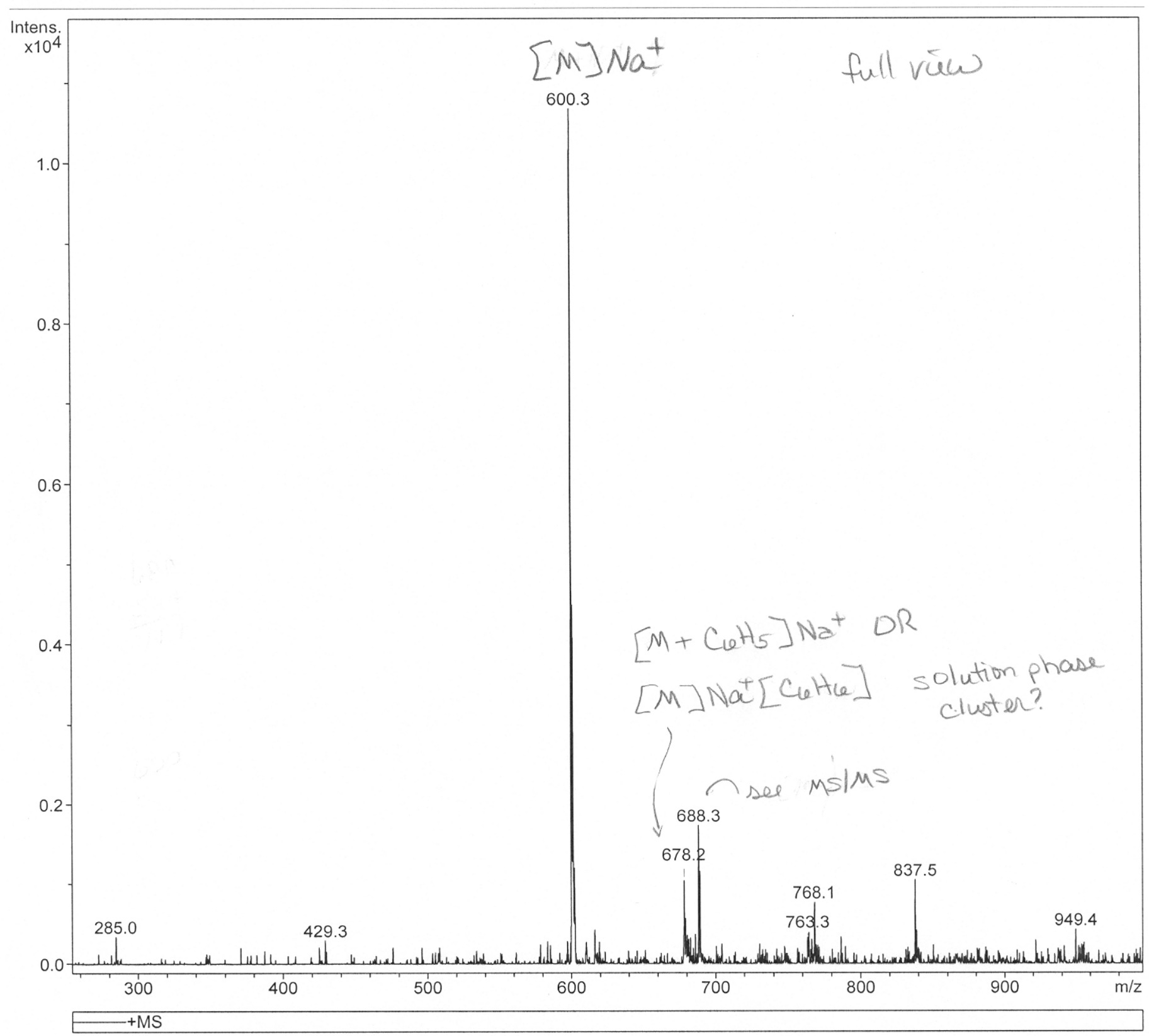




\section{Display Report}

\section{Analysis Info}

Analysis Name

Method

Sample Name

Comment
ES4042_4.d

DEFAULT.MS

research

Youngs/Paul Custer Paul 1 C33 H36 M3 Rh cationization confirmation

\section{Acquisition Date \\ Operator \\ Instrument}

02/18/04 16:23:48

Administrator

Esquire-LC_00143 120//5/8/150//600/20/100

\section{Acquisition Parameter}

Ion Source Type

Mass Range Mode

Capillary Exit

Accumulation Time

\section{ESI}

Std/Normal

87.4 Volt

$779 \mu \mathrm{s}$

Intens
$\times 10^{5}$

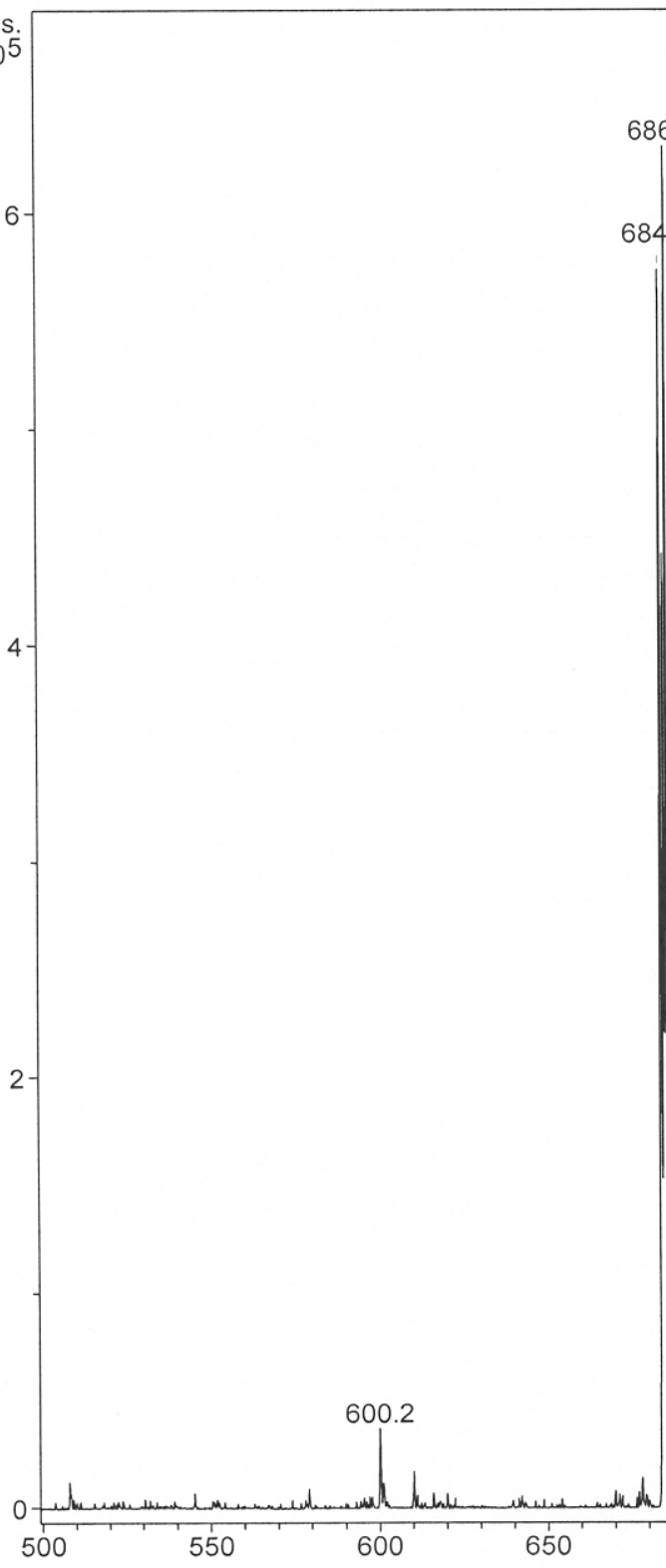

$\begin{array}{ll}\text { Ion Polarity } & \text { Positive } \\ \text { Scan Begin } & 150.00 \mathrm{~m} / \mathrm{z} \\ \text { Skim 1 } & 19.4 \text { Volt } \\ \text { Averages } & 40 \text { Spectra }\end{array}$

Alternating Ion Polarity

Scan End

Trap Drive

$1000.00 \mathrm{~m} / \mathrm{z}$

49.0

Off

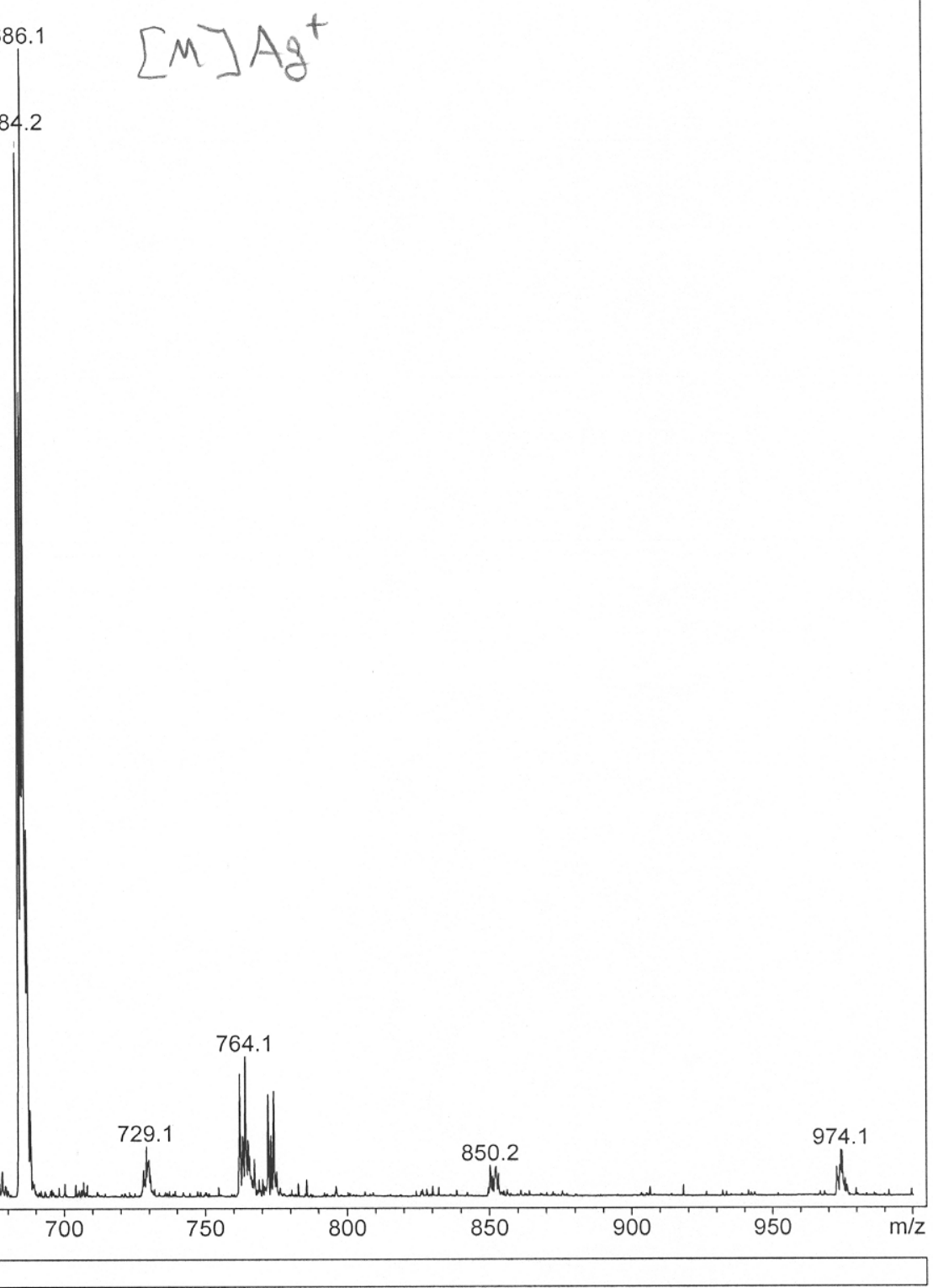




\section{Display Report}

\section{Analysis Info}

Analysis Name Method

Sample Name

Comment
ES423001.d

DEFAULT.MS

research

Youngs/Paul Custer 007A in CH3CN C 33 H 35 N 3 Rh B F 4 Ag in acetone 10/8/300/1544/10/140 enhanced resolution
Acquisition Date

Operator

Instrument
08/31/04 13:06:09

Administrator

Esquire-LC_00143

\section{Acquisition Parameter}

\section{Ion Source Type \\ ESI}

Mass Range Mode

Capillary Exit

Accumulation Time
Std/Enhanced

91.3 Volt

$123 \mu \mathrm{s}$

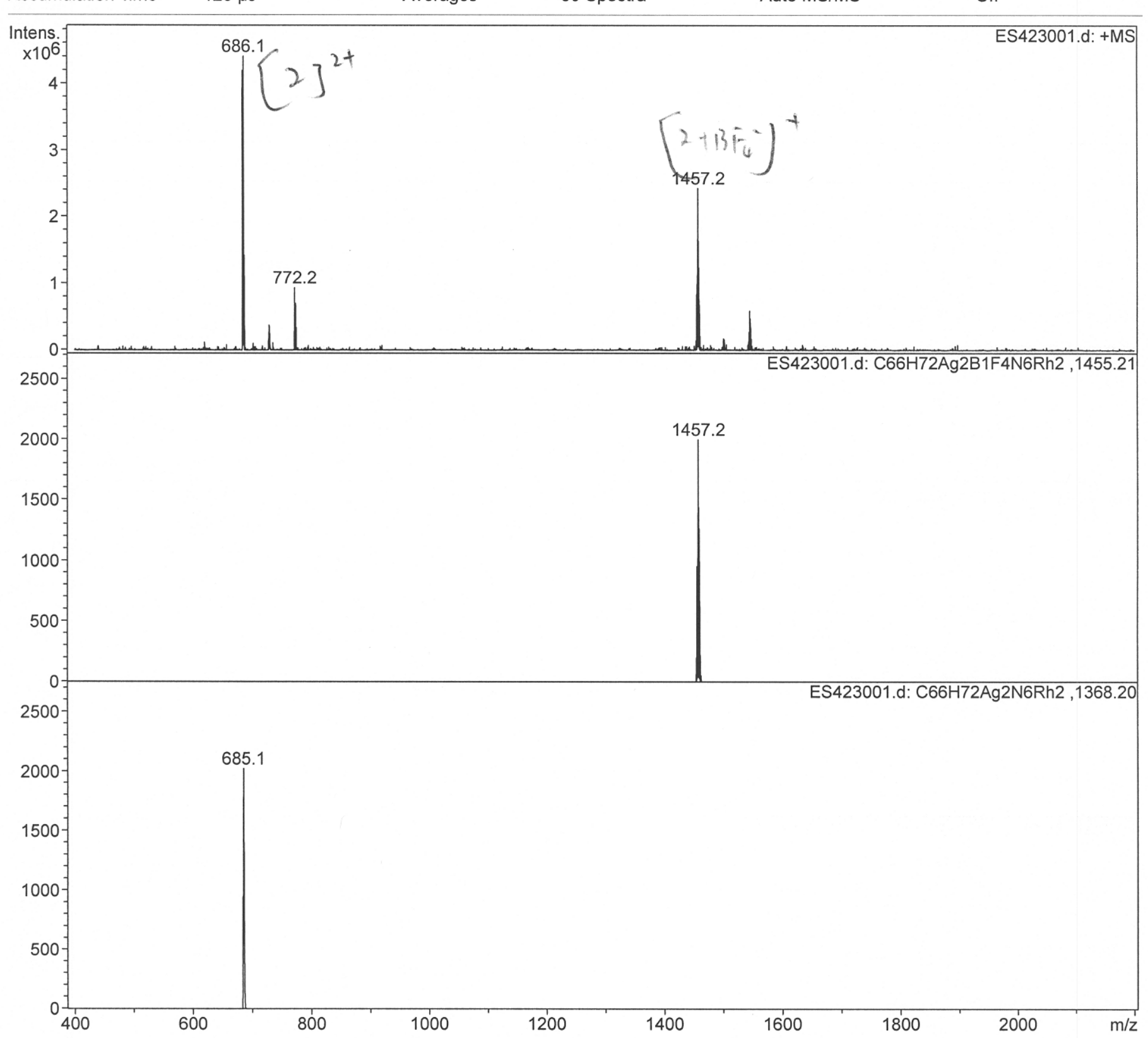




\section{Display Report}

Analysis Info

Analysis Name

Method

Sample Name

Comment
ES423001.d

DEFAULT.MS

research

Youngs/Paul Custer 007A in CH3CN C 33 H 35 N 3 Rh B F 4 Ag in acetone 10/8/300/1544/10/140 enhanced resolution
Acquisition Date

Operator

Instrument
08/31/04 13:06:09

Administrator

Esquire-LC_00143

\section{Acquisition Parameter}

Ion Source Type ESI

Mass Range Mode

Capillary Exit

Accumulation Time

Std/Enhanced

91.3 Volt

$123 \mu \mathrm{s}$

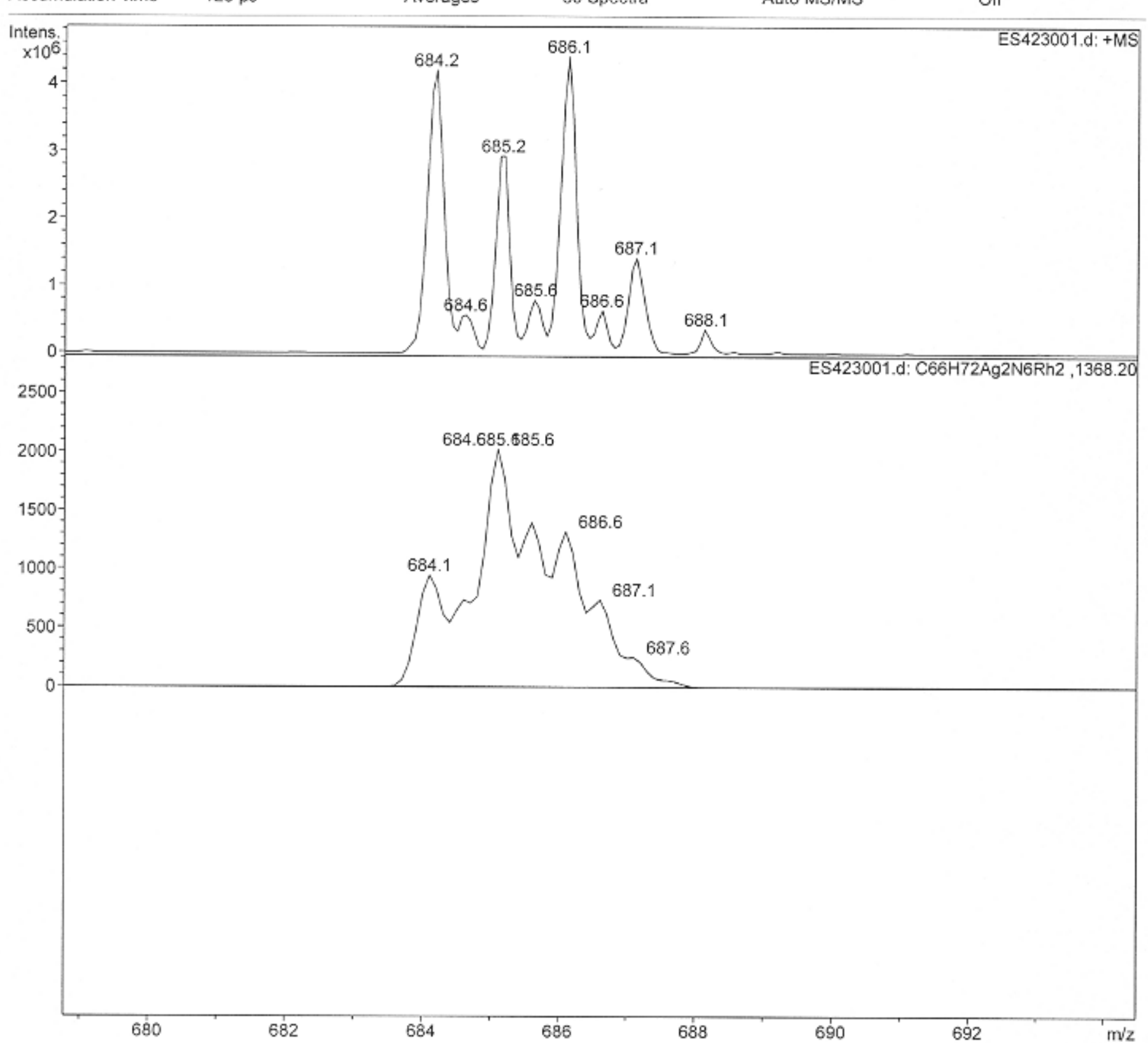




\section{Display Report}

\section{Analysis Info}

Analysis Name Method

Sample Name

Comment
ES423001.d

DEFAULT.MS

research

Youngs/Paul Custer 007A in CH3CN C 33 H 35 N 3 Rh B F 4 Ag in acetone 10/8/300/1544/10/140 enhanced resolution
Acquisition Date

Operator

08/31/04 13:06:09

Instrument

Administrator

Esquire-LC_00143

\section{Acquisition Parameter}

Ion Source Type ES

Mass Range Mode

Capillary Exit

Accumulation Time

\section{ESI}

Std/Enhanced

91.3 Volt

$123 \mu \mathrm{s}$

Intens.

$\mathrm{x} 10^{6}$

4

.

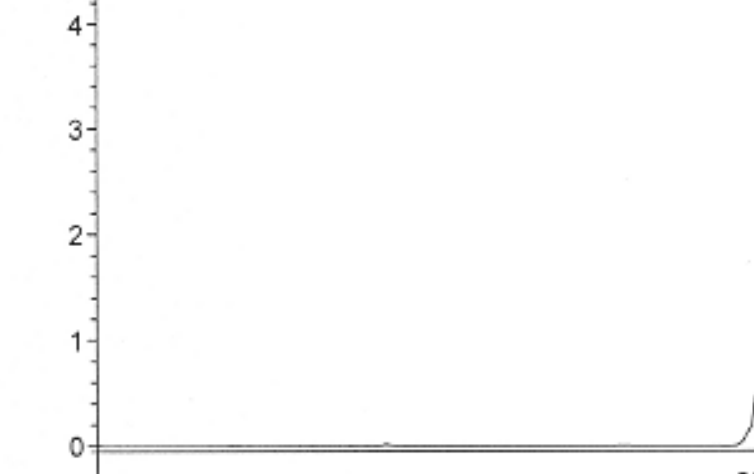

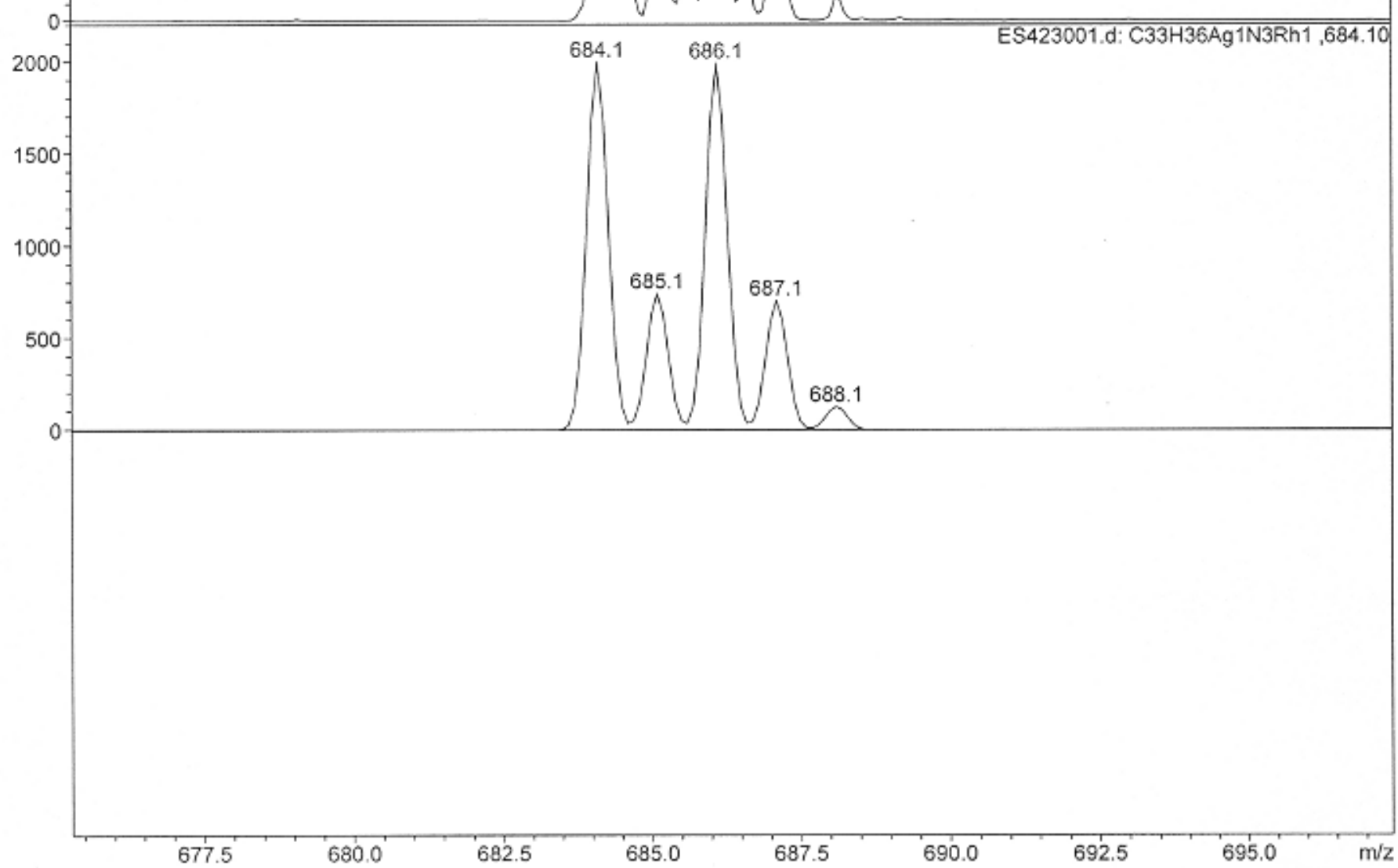




\section{X-ray Structure of 2 Asymmetric Unit}

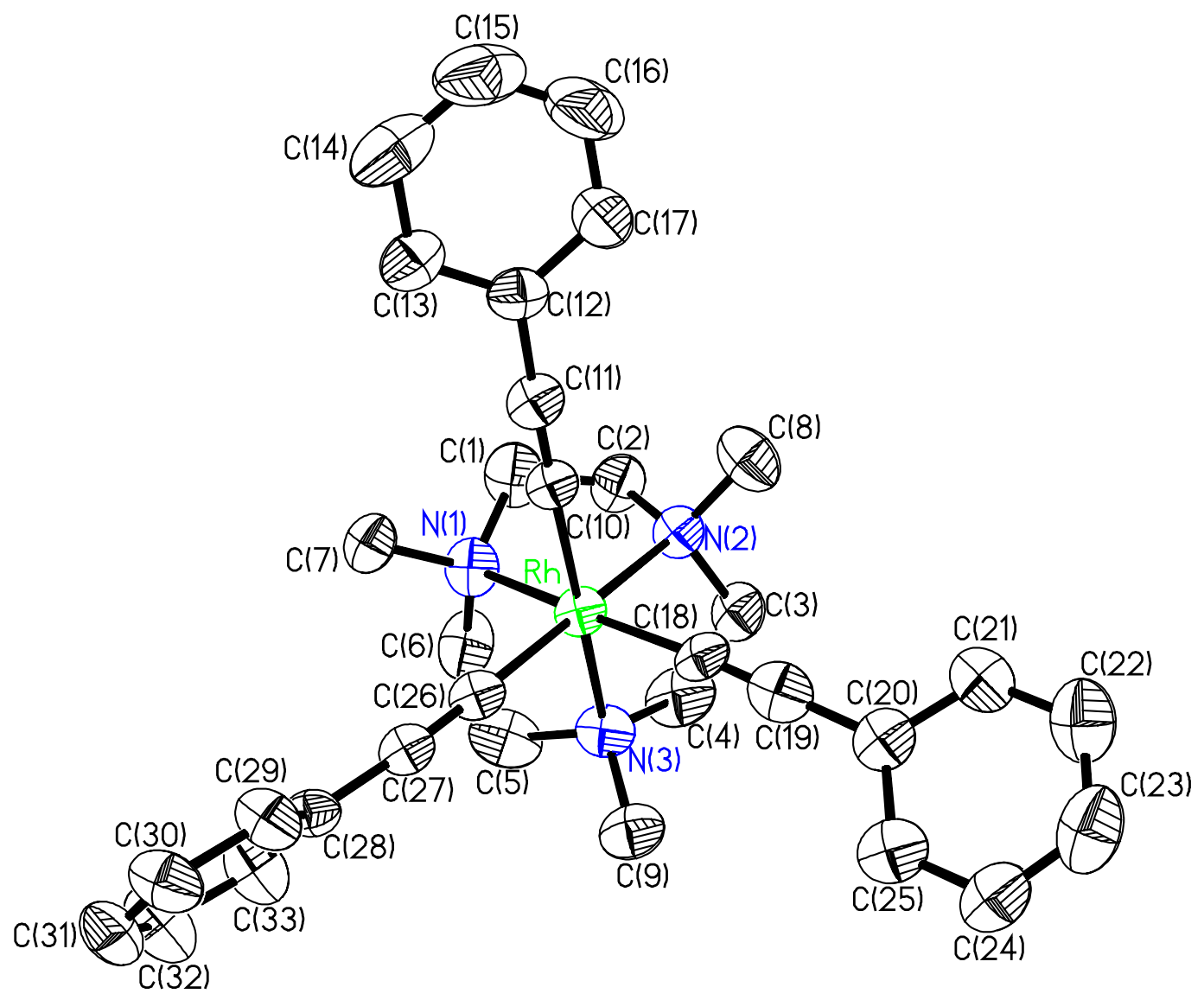




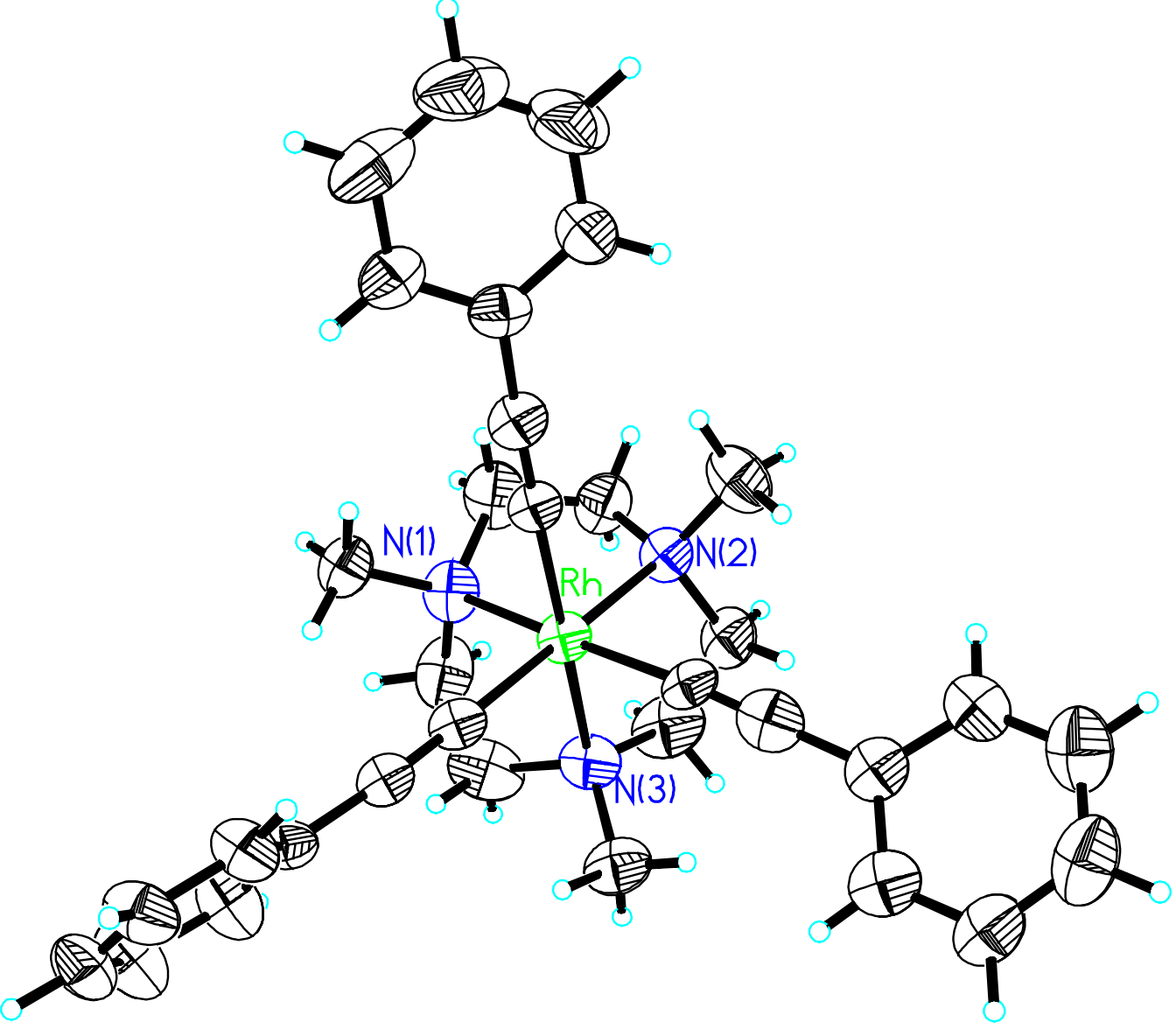


Table 1. Crystal data and structure refinement for $\mathbf{2}$.

Identification code

2

Empirical formula

C33 H36 N3 Rh

Formula weight

577.56

Temperature

100(2) K

Wavelength

$0.71073 \AA$

Crystal system

Monoclinic

Space group

Unit cell dimensions

P 21/c

$\mathrm{a}=11.4645(10) \AA \quad \alpha=90^{\circ}$.

$\mathrm{b}=17.7575(15) \AA \quad \beta=92.6900(10)^{\circ}$.

$\mathrm{c}=13.7191(11) \AA \quad \gamma=90^{\circ}$.

Volume

2789.9(4) $\AA^{3}$

$\mathrm{Z}$

4

Density (calculated)

$1.375 \mathrm{Mg} / \mathrm{m}^{3}$

Absorption coefficient

$0.638 \mathrm{~mm}^{-1}$

$\mathrm{F}(000)$

1200

Crystal size

$0.13 \times 0.08 \times 0.03 \mathrm{~mm}^{3}$

Theta range for data collection

1.78 to $27.51^{\circ}$.

Index ranges

$-14 \leq \mathrm{h} \leq 14,-22 \leq \mathrm{k} \leq 22,-17 \leq 1 \leq 17$

Reflections collected

24233

Independent reflections

$6381[\mathrm{R}(\mathrm{int})=0.0527]$

Completeness to theta $=27.51^{\circ}$

Absorption correction

$99.6 \%$

Max. and min. transmission

Semi-empirical from equivalents

0.9811 and 0.8934

Refinement method

Full-matrix least-squares on $\mathrm{F}^{2}$

Data / restraints / parameters

$6381 / 0 / 337$

Goodness-of-fit on $\mathrm{F}^{2}$

1.038

Final $\mathrm{R}$ indices [I $>2 \operatorname{sigma}(\mathrm{I})]$

$\mathrm{R} 1=0.0534, \mathrm{wR} 2=0.1277$

$\mathrm{R}$ indices (all data)

$\mathrm{R} 1=0.0951, w \mathrm{R} 2=0.1456$

Largest diff. peak and hole

1.398 and -0.356 e. $\AA^{-3}$ 
Table 2. Atomic coordinates ( $\mathrm{x} 10^{4}$ ) and equivalent isotropic displacement parameters $\left(\AA^{2} \times 10^{3}\right.$ ) for 2 . $U(e q)$ is defined as one third of the trace of the orthogonalized $U^{\mathrm{ij}}$ tensor.

\begin{tabular}{|c|c|c|c|c|}
\hline & $\mathrm{x}$ & $\mathrm{y}$ & $\mathrm{z}$ & $\mathrm{U}(\mathrm{eq})$ \\
\hline $\mathrm{Rh}$ & $5855(1)$ & 6909(1) & 9001(1) & $37(1)$ \\
\hline $\mathrm{N}(1)$ & $5385(4)$ & 5904(2) & $8189(2)$ & $49(1)$ \\
\hline $\mathrm{N}(2)$ & 4967(3) & 7441(2) & $7768(2)$ & $43(1)$ \\
\hline $\mathrm{N}(3)$ & 7298(4) & $6909(2)$ & $8058(3)$ & $49(1)$ \\
\hline $\mathrm{C}(1)$ & $4289(5)$ & 6122(3) & $7616(4)$ & $67(2)$ \\
\hline $\mathrm{C}(2)$ & $4385(5)$ & 6858(3) & 7135(4) & $60(1)$ \\
\hline $\mathrm{C}(3)$ & $5921(5)$ & $7816(3)$ & $7240(4)$ & $56(1)$ \\
\hline $\mathrm{C}(4)$ & $6954(5)$ & 7341(4) & 7151(4) & $73(2)$ \\
\hline $\mathrm{C}(5)$ & $7431(5)$ & 6078(4) & $7847(4)$ & $70(2)$ \\
\hline$C(6)$ & $6336(5)$ & $5702(3)$ & $7544(4)$ & $67(2)$ \\
\hline $\mathrm{C}(7)$ & $5095(5)$ & $5252(3)$ & 8791(3) & $52(1)$ \\
\hline$C(8)$ & $4119(5)$ & $8027(3)$ & $8036(4)$ & $66(2)$ \\
\hline$C(9)$ & $8411(4)$ & 7190(3) & $8523(4)$ & $59(1)$ \\
\hline$C(10)$ & $4432(4)$ & 6866(3) & $9781(3)$ & $37(1)$ \\
\hline $\mathrm{C}(11)$ & $3536(4)$ & 6832(3) & 10171(3) & $46(1)$ \\
\hline$C(12)$ & $2419(4)$ & 6808(3) & $10619(4)$ & $47(1)$ \\
\hline$C(13)$ & $2105(5)$ & 6196(3) & 11184(4) & $58(1)$ \\
\hline$C(14)$ & 1023(6) & $6169(4)$ & $11607(5)$ & $85(2)$ \\
\hline$C(15)$ & $262(6)$ & $6741(5)$ & $11448(7)$ & $104(3)$ \\
\hline$C(16)$ & $544(6)$ & $7347(4)$ & $10919(7)$ & $109(3)$ \\
\hline $\mathrm{C}(17)$ & $1622(5)$ & 7389(3) & $10500(6)$ & $80(2)$ \\
\hline $\mathrm{C}(18)$ & $6314(4)$ & 7907(3) & $9596(3)$ & $40(1)$ \\
\hline$C(19)$ & $6674(5)$ & $8488(3)$ & $9773(3)$ & $53(1)$ \\
\hline $\mathrm{C}(20)$ & 7143(4) & 9239(3) & 10018(3) & $50(1)$ \\
\hline $\mathrm{C}(21)$ & $6504(5)$ & 9896(3) & $9853(4)$ & $56(1)$ \\
\hline $\mathrm{C}(22)$ & $6894(6)$ & $10590(4)$ & $10134(4)$ & $74(2)$ \\
\hline $\mathrm{C}(23)$ & $7965(6)$ & $10657(4)$ & $10576(4)$ & $77(2)$ \\
\hline $\mathrm{C}(24)$ & $8669(5)$ & $10047(3)$ & $10724(4)$ & $64(2)$ \\
\hline $\mathrm{C}(25)$ & $8267(5)$ & 9339(3) & 10439(3) & $59(1)$ \\
\hline$C(26)$ & $6741(4)$ & $6350(3)$ & 10039(3) & $38(1)$ \\
\hline $\mathrm{C}(27)$ & 7295(4) & 5964(3) & $10605(3)$ & $39(1)$ \\
\hline
\end{tabular}




\begin{tabular}{lllll}
$\mathrm{C}(28)$ & $7928(4)$ & $5428(3)$ & $11216(3)$ & $41(1)$ \\
$\mathrm{C}(29)$ & $7866(4)$ & $5403(3)$ & $12221(3)$ & $52(1)$ \\
$\mathrm{C}(30)$ & $8444(4)$ & $4851(3)$ & $12770(4)$ & $59(1)$ \\
$\mathrm{C}(31)$ & $9080(5)$ & $4310(3)$ & $12326(4)$ & $61(1)$ \\
$\mathrm{C}(32)$ & $9159(5)$ & $4332(4)$ & $11323(4)$ & $74(2)$ \\
$\mathrm{C}(33)$ & $8610(5)$ & $4888(3)$ & $10783(4)$ & $62(2)$ \\
\hline
\end{tabular}




\begin{tabular}{|c|c|c|c|}
\hline \multirow{2}{*}{\multicolumn{2}{|c|}{ Table 3. Bond lengths $[\AA \AA]$ and angles $\left[{ }^{\circ}\right]$ for 2.}} & \multirow{2}{*}{$\begin{array}{l}\mathrm{C}(8)-\mathrm{H}(8 \mathrm{~A}) \\
\mathrm{C}(8)-\mathrm{H}(8 \mathrm{~B})\end{array}$} & \multirow{2}{*}{$\begin{array}{l}0.9800 \\
0.9800\end{array}$} \\
\hline & & & \\
\hline $\mathrm{Rh}-\mathrm{C}(26)$ & $1.977(5)$ & $\mathrm{C}(8)-\mathrm{H}(8 \mathrm{C})$ & 0.9800 \\
\hline $\mathrm{Rh}-\mathrm{C}(10)$ & $1.995(4)$ & $\mathrm{C}(9)-\mathrm{H}(9 \mathrm{~A})$ & 0.9800 \\
\hline $\mathrm{Rh}-\mathrm{C}(18)$ & $2.010(5)$ & $\mathrm{C}(9)-\mathrm{H}(9 \mathrm{~B})$ & 0.9800 \\
\hline Rh-N(3) & $2.147(4)$ & $\mathrm{C}(9)-\mathrm{H}(9 \mathrm{C})$ & 0.9800 \\
\hline $\mathrm{Rh}-\mathrm{N}(2)$ & $2.151(4)$ & $C(10)-C(11)$ & $1.181(6)$ \\
\hline $\mathrm{Rh}-\mathrm{N}(1)$ & $2.159(4)$ & $\mathrm{C}(11)-\mathrm{C}(12)$ & $1.446(7)$ \\
\hline $\mathrm{N}(1)-\mathrm{C}(7)$ & $1.471(6)$ & $\mathrm{C}(12)-\mathrm{C}(17)$ & $1.381(7)$ \\
\hline $\mathrm{N}(1)-\mathrm{C}(6)$ & $1.480(6)$ & $C(12)-C(13)$ & $1.393(7)$ \\
\hline $\mathrm{N}(1)-\mathrm{C}(1)$ & $1.500(6)$ & $\mathrm{C}(13)-\mathrm{C}(14)$ & $1.395(8)$ \\
\hline $\mathrm{N}(2)-\mathrm{C}(8)$ & $1.483(6)$ & $\mathrm{C}(13)-\mathrm{H}(13)$ & 0.9500 \\
\hline $\mathrm{N}(2)-\mathrm{C}(2)$ & $1.489(6)$ & $C(14)-C(15)$ & $1.350(10)$ \\
\hline $\mathrm{N}(2)-\mathrm{C}(3)$ & $1.496(6)$ & $\mathrm{C}(14)-\mathrm{H}(14)$ & 0.9500 \\
\hline$N(3)-C(9)$ & $1.486(7)$ & $C(15)-C(16)$ & $1.347(11)$ \\
\hline $\mathrm{N}(3)-\mathrm{C}(4)$ & $1.499(6)$ & $\mathrm{C}(15)-\mathrm{H}(15)$ & 0.9500 \\
\hline $\mathrm{N}(3)-\mathrm{C}(5)$ & $1.511(7)$ & $C(16)-C(17)$ & $1.389(9)$ \\
\hline $\mathrm{C}(1)-\mathrm{C}(2)$ & $1.469(7)$ & $\mathrm{C}(16)-\mathrm{H}(16)$ & 0.9500 \\
\hline $\mathrm{C}(1)-\mathrm{H}(1 \mathrm{~A})$ & 0.9900 & $\mathrm{C}(17)-\mathrm{H}(17)$ & 0.9500 \\
\hline $\mathrm{C}(1)-\mathrm{H}(1 \mathrm{~B})$ & 0.9900 & $C(18)-C(19)$ & $1.135(7)$ \\
\hline $\mathrm{C}(2)-\mathrm{H}(2 \mathrm{~A})$ & 0.9900 & $C(19)-C(20)$ & $1.470(8)$ \\
\hline $\mathrm{C}(2)-\mathrm{H}(2 \mathrm{~B})$ & 0.9900 & $C(20)-C(21)$ & $1.390(7)$ \\
\hline $\mathrm{C}(3)-\mathrm{C}(4)$ & $1.463(8)$ & $C(20)-C(25)$ & $1.399(7)$ \\
\hline $\mathrm{C}(3)-\mathrm{H}(3 \mathrm{~A})$ & 0.9900 & $C(21)-C(22)$ & $1.359(8)$ \\
\hline $\mathrm{C}(3)-\mathrm{H}(3 \mathrm{~B})$ & 0.9900 & $\mathrm{C}(21)-\mathrm{H}(21)$ & 0.9500 \\
\hline $\mathrm{C}(4)-\mathrm{H}(4 \mathrm{~A})$ & 0.9900 & $C(22)-C(23)$ & $1.350(9)$ \\
\hline $\mathrm{C}(4)-\mathrm{H}(4 \mathrm{~B})$ & 0.9900 & $\mathrm{C}(22)-\mathrm{H}(22)$ & 0.9500 \\
\hline$C(5)-C(6)$ & $1.466(8)$ & $C(23)-C(24)$ & $1.360(8)$ \\
\hline $\mathrm{C}(5)-\mathrm{H}(5 \mathrm{~A})$ & 0.9900 & $\mathrm{C}(23)-\mathrm{H}(23)$ & 0.9500 \\
\hline $\mathrm{C}(5)-\mathrm{H}(5 \mathrm{~B})$ & 0.9900 & $C(24)-C(25)$ & $1.390(8)$ \\
\hline $\mathrm{C}(6)-\mathrm{H}(6 \mathrm{~A})$ & 0.9900 & $\mathrm{C}(24)-\mathrm{H}(24)$ & 0.9500 \\
\hline $\mathrm{C}(6)-\mathrm{H}(6 \mathrm{~B})$ & 0.9900 & $\mathrm{C}(25)-\mathrm{H}(25)$ & 0.9500 \\
\hline $\mathrm{C}(7)-\mathrm{H}(7 \mathrm{~A})$ & 0.9800 & $C(26)-C(27)$ & $1.197(6)$ \\
\hline $\mathrm{C}(7)-\mathrm{H}(7 \mathrm{~B})$ & 0.9800 & $\mathrm{C}(27)-\mathrm{C}(28)$ & $1.441(6)$ \\
\hline $\mathrm{C}(7)-\mathrm{H}(7 \mathrm{C})$ & 0.9800 & $\mathrm{C}(28)-\mathrm{C}(29)$ & $1.386(6)$ \\
\hline
\end{tabular}




\begin{tabular}{|c|c|c|c|}
\hline$C(28)-C(33)$ & $1.387(7)$ & $\mathrm{C}(2)-\mathrm{N}(2)-\mathrm{Rh}$ & $109.6(3)$ \\
\hline $\mathrm{C}(29)-\mathrm{C}(30)$ & $1.384(7)$ & $\mathrm{C}(3)-\mathrm{N}(2)-\mathrm{Rh}$ & $104.3(3)$ \\
\hline $\mathrm{C}(29)-\mathrm{H}(29)$ & 0.9500 & $\mathrm{C}(9)-\mathrm{N}(3)-\mathrm{C}(4)$ & $111.8(4)$ \\
\hline $\mathrm{C}(30)-\mathrm{C}(31)$ & $1.366(7)$ & $\mathrm{C}(9)-\mathrm{N}(3)-\mathrm{C}(5)$ & $108.5(4)$ \\
\hline $\mathrm{C}(30)-\mathrm{H}(30)$ & 0.9500 & $\mathrm{C}(4)-\mathrm{N}(3)-\mathrm{C}(5)$ & 111.5(4) \\
\hline$C(31)-C(32)$ & $1.383(7)$ & $\mathrm{C}(9)-\mathrm{N}(3)-\mathrm{Rh}$ & $114.3(3)$ \\
\hline $\mathrm{C}(31)-\mathrm{H}(31)$ & 0.9500 & $\mathrm{C}(4)-\mathrm{N}(3)-\mathrm{Rh}$ & $108.6(3)$ \\
\hline$C(32)-C(33)$ & $1.371(7)$ & $\mathrm{C}(5)-\mathrm{N}(3)-\mathrm{Rh}$ & $101.7(3)$ \\
\hline $\mathrm{C}(32)-\mathrm{H}(32)$ & 0.9500 & $\mathrm{C}(2)-\mathrm{C}(1)-\mathrm{N}(1)$ & $112.7(5)$ \\
\hline \multirow[t]{2}{*}{$\mathrm{C}(33)-\mathrm{H}(33)$} & 0.9500 & $\mathrm{C}(2)-\mathrm{C}(1)-\mathrm{H}(1 \mathrm{~A})$ & 109.1 \\
\hline & & $\mathrm{N}(1)-\mathrm{C}(1)-\mathrm{H}(1 \mathrm{~A})$ & 109.1 \\
\hline $\mathrm{C}(26)-\mathrm{Rh}-\mathrm{C}(10)$ & $90.00(17)$ & $\mathrm{C}(2)-\mathrm{C}(1)-\mathrm{H}(1 \mathrm{~B})$ & 109.1 \\
\hline $\mathrm{C}(26)-\mathrm{Rh}-\mathrm{C}(18)$ & $91.94(17)$ & $\mathrm{N}(1)-\mathrm{C}(1)-\mathrm{H}(1 \mathrm{~B})$ & 109.1 \\
\hline $\mathrm{C}(10)-\mathrm{Rh}-\mathrm{C}(18)$ & $91.16(18)$ & $\mathrm{H}(1 \mathrm{~A})-\mathrm{C}(1)-\mathrm{H}(1 \mathrm{~B})$ & 107.8 \\
\hline $\mathrm{C}(26)-\mathrm{Rh}-\mathrm{N}(3)$ & $92.84(16)$ & $\mathrm{C}(1)-\mathrm{C}(2)-\mathrm{N}(2)$ & $113.4(4)$ \\
\hline $\mathrm{C}(10)-\mathrm{Rh}-\mathrm{N}(3)$ & 174.93(16) & $\mathrm{C}(1)-\mathrm{C}(2)-\mathrm{H}(2 \mathrm{~A})$ & 108.9 \\
\hline $\mathrm{C}(18)-\mathrm{Rh}-\mathrm{N}(3)$ & $92.95(17)$ & $\mathrm{N}(2)-\mathrm{C}(2)-\mathrm{H}(2 \mathrm{~A})$ & 108.9 \\
\hline $\mathrm{C}(26)-\mathrm{Rh}-\mathrm{N}(2)$ & $174.21(15)$ & $\mathrm{C}(1)-\mathrm{C}(2)-\mathrm{H}(2 \mathrm{~B})$ & 108.9 \\
\hline $\mathrm{C}(10)-\mathrm{Rh}-\mathrm{N}(2)$ & $94.00(16)$ & $\mathrm{N}(2)-\mathrm{C}(2)-\mathrm{H}(2 \mathrm{~B})$ & 108.9 \\
\hline $\mathrm{C}(18)-\mathrm{Rh}-\mathrm{N}(2)$ & $92.17(15)$ & $\mathrm{H}(2 \mathrm{~A})-\mathrm{C}(2)-\mathrm{H}(2 \mathrm{~B})$ & 107.7 \\
\hline $\mathrm{N}(3)-\mathrm{Rh}-\mathrm{N}(2)$ & $82.88(15)$ & $\mathrm{C}(4)-\mathrm{C}(3)-\mathrm{N}(2)$ & 113.3(4) \\
\hline $\mathrm{C}(26)-\mathrm{Rh}-\mathrm{N}(1)$ & $93.69(16)$ & $\mathrm{C}(4)-\mathrm{C}(3)-\mathrm{H}(3 \mathrm{~A})$ & 108.9 \\
\hline $\mathrm{C}(10)-\mathrm{Rh}-\mathrm{N}(1)$ & $93.11(16)$ & $\mathrm{N}(2)-\mathrm{C}(3)-\mathrm{H}(3 \mathrm{~A})$ & 108.9 \\
\hline $\mathrm{C}(18)-\mathrm{Rh}-\mathrm{N}(1)$ & $172.93(15)$ & $\mathrm{C}(4)-\mathrm{C}(3)-\mathrm{H}(3 \mathrm{~B})$ & 108.9 \\
\hline $\mathrm{N}(3)-\mathrm{Rh}-\mathrm{N}(1)$ & $82.51(15)$ & $\mathrm{N}(2)-\mathrm{C}(3)-\mathrm{H}(3 \mathrm{~B})$ & 108.9 \\
\hline $\mathrm{N}(2)-\mathrm{Rh}-\mathrm{N}(1)$ & $81.92(14)$ & $\mathrm{H}(3 \mathrm{~A})-\mathrm{C}(3)-\mathrm{H}(3 \mathrm{~B})$ & 107.7 \\
\hline $\mathrm{C}(7)-\mathrm{N}(1)-\mathrm{C}(6)$ & $109.6(4)$ & $\mathrm{C}(3)-\mathrm{C}(4)-\mathrm{N}(3)$ & 114.1(4) \\
\hline $\mathrm{C}(7)-\mathrm{N}(1)-\mathrm{C}(1)$ & 107.0(4) & $\mathrm{C}(3)-\mathrm{C}(4)-\mathrm{H}(4 \mathrm{~A})$ & 108.7 \\
\hline $\mathrm{C}(6)-\mathrm{N}(1)-\mathrm{C}(1)$ & $111.9(4)$ & $\mathrm{N}(3)-\mathrm{C}(4)-\mathrm{H}(4 \mathrm{~A})$ & 108.7 \\
\hline $\mathrm{C}(7)-\mathrm{N}(1)-\mathrm{Rh}$ & $114.8(3)$ & $\mathrm{C}(3)-\mathrm{C}(4)-\mathrm{H}(4 \mathrm{~B})$ & 108.7 \\
\hline$C(6)-N(1)-R h$ & $109.6(3)$ & $\mathrm{N}(3)-\mathrm{C}(4)-\mathrm{H}(4 \mathrm{~B})$ & 108.7 \\
\hline $\mathrm{C}(1)-\mathrm{N}(1)-\mathrm{Rh}$ & $103.9(3)$ & $\mathrm{H}(4 \mathrm{~A})-\mathrm{C}(4)-\mathrm{H}(4 \mathrm{~B})$ & 107.6 \\
\hline $\mathrm{C}(8)-\mathrm{N}(2)-\mathrm{C}(2)$ & $110.6(4)$ & $\mathrm{C}(6)-\mathrm{C}(5)-\mathrm{N}(3)$ & $114.0(5)$ \\
\hline $\mathrm{C}(8)-\mathrm{N}(2)-\mathrm{C}(3)$ & $108.1(4)$ & $\mathrm{C}(6)-\mathrm{C}(5)-\mathrm{H}(5 \mathrm{~A})$ & 108.8 \\
\hline $\mathrm{C}(2)-\mathrm{N}(2)-\mathrm{C}(3)$ & $110.2(4)$ & $\mathrm{N}(3)-\mathrm{C}(5)-\mathrm{H}(5 \mathrm{~A})$ & 108.8 \\
\hline $\mathrm{C}(8)-\mathrm{N}(2)-\mathrm{Rh}$ & $113.9(3)$ & $\mathrm{C}(6)-\mathrm{C}(5)-\mathrm{H}(5 \mathrm{~B})$ & 108.8 \\
\hline
\end{tabular}




\begin{tabular}{|c|c|c|c|}
\hline $\mathrm{N}(3)-\mathrm{C}(5)-\mathrm{H}(5 \mathrm{~B})$ & 108.8 & $\mathrm{C}(13)-\mathrm{C}(14)-\mathrm{H}(14)$ & 120.4 \\
\hline $\mathrm{H}(5 \mathrm{~A})-\mathrm{C}(5)-\mathrm{H}(5 \mathrm{~B})$ & 107.7 & $C(16)-C(15)-C(14)$ & $121.2(7)$ \\
\hline$C(5)-C(6)-N(1)$ & $111.4(4)$ & $\mathrm{C}(16)-\mathrm{C}(15)-\mathrm{H}(15)$ & 119.4 \\
\hline $\mathrm{C}(5)-\mathrm{C}(6)-\mathrm{H}(6 \mathrm{~A})$ & 109.3 & $\mathrm{C}(14)-\mathrm{C}(15)-\mathrm{H}(15)$ & 119.4 \\
\hline $\mathrm{N}(1)-\mathrm{C}(6)-\mathrm{H}(6 \mathrm{~A})$ & 109.3 & $C(15)-C(16)-C(17)$ & $120.4(7)$ \\
\hline $\mathrm{C}(5)-\mathrm{C}(6)-\mathrm{H}(6 \mathrm{~B})$ & 109.3 & $\mathrm{C}(15)-\mathrm{C}(16)-\mathrm{H}(16)$ & 119.8 \\
\hline $\mathrm{N}(1)-\mathrm{C}(6)-\mathrm{H}(6 \mathrm{~B})$ & 109.3 & $\mathrm{C}(17)-\mathrm{C}(16)-\mathrm{H}(16)$ & 119.8 \\
\hline $\mathrm{H}(6 \mathrm{~A})-\mathrm{C}(6)-\mathrm{H}(6 \mathrm{~B})$ & 108.0 & $\mathrm{C}(12)-\mathrm{C}(17)-\mathrm{C}(16)$ & $120.5(6)$ \\
\hline $\mathrm{N}(1)-\mathrm{C}(7)-\mathrm{H}(7 \mathrm{~A})$ & 109.5 & $\mathrm{C}(12)-\mathrm{C}(17)-\mathrm{H}(17)$ & 119.7 \\
\hline $\mathrm{N}(1)-\mathrm{C}(7)-\mathrm{H}(7 \mathrm{~B})$ & 109.5 & $\mathrm{C}(16)-\mathrm{C}(17)-\mathrm{H}(17)$ & 119.7 \\
\hline $\mathrm{H}(7 \mathrm{~A})-\mathrm{C}(7)-\mathrm{H}(7 \mathrm{~B})$ & 109.5 & $\mathrm{C}(19)-\mathrm{C}(18)-\mathrm{Rh}$ & $167.1(4)$ \\
\hline $\mathrm{N}(1)-\mathrm{C}(7)-\mathrm{H}(7 \mathrm{C})$ & 109.5 & $C(18)-C(19)-C(20)$ & $179.1(5)$ \\
\hline $\mathrm{H}(7 \mathrm{~A})-\mathrm{C}(7)-\mathrm{H}(7 \mathrm{C})$ & 109.5 & $C(21)-C(20)-C(25)$ & $115.4(5)$ \\
\hline $\mathrm{H}(7 \mathrm{~B})-\mathrm{C}(7)-\mathrm{H}(7 \mathrm{C})$ & 109.5 & $C(21)-C(20)-C(19)$ & $122.7(5)$ \\
\hline $\mathrm{N}(2)-\mathrm{C}(8)-\mathrm{H}(8 \mathrm{~A})$ & 109.5 & $C(25)-C(20)-C(19)$ & $121.9(5)$ \\
\hline $\mathrm{N}(2)-\mathrm{C}(8)-\mathrm{H}(8 \mathrm{~B})$ & 109.5 & $C(22)-C(21)-C(20)$ & $123.4(6)$ \\
\hline $\mathrm{H}(8 \mathrm{~A})-\mathrm{C}(8)-\mathrm{H}(8 \mathrm{~B})$ & 109.5 & $\mathrm{C}(22)-\mathrm{C}(21)-\mathrm{H}(21)$ & 118.3 \\
\hline $\mathrm{N}(2)-\mathrm{C}(8)-\mathrm{H}(8 \mathrm{C})$ & 109.5 & $\mathrm{C}(20)-\mathrm{C}(21)-\mathrm{H}(21)$ & 118.3 \\
\hline $\mathrm{H}(8 \mathrm{~A})-\mathrm{C}(8)-\mathrm{H}(8 \mathrm{C})$ & 109.5 & $\mathrm{C}(23)-\mathrm{C}(22)-\mathrm{C}(21)$ & $119.2(6)$ \\
\hline $\mathrm{H}(8 \mathrm{~B})-\mathrm{C}(8)-\mathrm{H}(8 \mathrm{C})$ & 109.5 & $\mathrm{C}(23)-\mathrm{C}(22)-\mathrm{H}(22)$ & 120.4 \\
\hline $\mathrm{N}(3)-\mathrm{C}(9)-\mathrm{H}(9 \mathrm{~A})$ & 109.5 & $\mathrm{C}(21)-\mathrm{C}(22)-\mathrm{H}(22)$ & 120.4 \\
\hline $\mathrm{N}(3)-\mathrm{C}(9)-\mathrm{H}(9 \mathrm{~B})$ & 109.5 & $\mathrm{C}(22)-\mathrm{C}(23)-\mathrm{C}(24)$ & $121.1(6)$ \\
\hline $\mathrm{H}(9 \mathrm{~A})-\mathrm{C}(9)-\mathrm{H}(9 \mathrm{~B})$ & 109.5 & $\mathrm{C}(22)-\mathrm{C}(23)-\mathrm{H}(23)$ & 119.4 \\
\hline $\mathrm{N}(3)-\mathrm{C}(9)-\mathrm{H}(9 \mathrm{C})$ & 109.5 & $\mathrm{C}(24)-\mathrm{C}(23)-\mathrm{H}(23)$ & 119.4 \\
\hline $\mathrm{H}(9 \mathrm{~A})-\mathrm{C}(9)-\mathrm{H}(9 \mathrm{C})$ & 109.5 & $\mathrm{C}(23)-\mathrm{C}(24)-\mathrm{C}(25)$ & $119.5(6)$ \\
\hline $\mathrm{H}(9 \mathrm{~B})-\mathrm{C}(9)-\mathrm{H}(9 \mathrm{C})$ & 109.5 & $\mathrm{C}(23)-\mathrm{C}(24)-\mathrm{H}(24)$ & 120.2 \\
\hline $\mathrm{C}(11)-\mathrm{C}(10)-\mathrm{Rh}$ & $174.4(4)$ & $\mathrm{C}(25)-\mathrm{C}(24)-\mathrm{H}(24)$ & 120.2 \\
\hline$C(10)-C(11)-C(12)$ & $177.8(5)$ & $\mathrm{C}(24)-\mathrm{C}(25)-\mathrm{C}(20)$ & $121.2(6)$ \\
\hline $\mathrm{C}(17)-\mathrm{C}(12)-\mathrm{C}(13)$ & $117.5(5)$ & $\mathrm{C}(24)-\mathrm{C}(25)-\mathrm{H}(25)$ & 119.4 \\
\hline $\mathrm{C}(17)-\mathrm{C}(12)-\mathrm{C}(11)$ & $121.5(5)$ & $\mathrm{C}(20)-\mathrm{C}(25)-\mathrm{H}(25)$ & 119.4 \\
\hline $\mathrm{C}(13)-\mathrm{C}(12)-\mathrm{C}(11)$ & $121.0(5)$ & $C(27)-C(26)-R h$ & 174.1(4) \\
\hline $\mathrm{C}(12)-\mathrm{C}(13)-\mathrm{C}(14)$ & $121.0(6)$ & $\mathrm{C}(26)-\mathrm{C}(27)-\mathrm{C}(28)$ & $173.6(5)$ \\
\hline $\mathrm{C}(12)-\mathrm{C}(13)-\mathrm{H}(13)$ & 119.5 & $\mathrm{C}(29)-\mathrm{C}(28)-\mathrm{C}(33)$ & $117.4(4)$ \\
\hline $\mathrm{C}(14)-\mathrm{C}(13)-\mathrm{H}(13)$ & 119.5 & $\mathrm{C}(29)-\mathrm{C}(28)-\mathrm{C}(27)$ & $123.5(4)$ \\
\hline$C(15)-C(14)-C(13)$ & $119.3(7)$ & $\mathrm{C}(33)-\mathrm{C}(28)-\mathrm{C}(27)$ & 119.1(4) \\
\hline $\mathrm{C}(15)-\mathrm{C}(14)-\mathrm{H}(14)$ & 120.4 & $\mathrm{C}(30)-\mathrm{C}(29)-\mathrm{C}(28)$ & $121.2(5)$ \\
\hline
\end{tabular}




$\begin{array}{llll}\mathrm{C}(30)-\mathrm{C}(29)-\mathrm{H}(29) & 119.4 & \mathrm{C}(33)-\mathrm{C}(32)-\mathrm{H}(32) & 119.7 \\ \mathrm{C}(28)-\mathrm{C}(29)-\mathrm{H}(29) & 119.4 & \mathrm{C}(31)-\mathrm{C}(32)-\mathrm{H}(32) & 119.7 \\ \mathrm{C}(31)-\mathrm{C}(30)-\mathrm{C}(29) & 120.4(5) & \mathrm{C}(32)-\mathrm{C}(33)-\mathrm{C}(28) & 121.4(5) \\ \mathrm{C}(31)-\mathrm{C}(30)-\mathrm{H}(30) & 119.8 & \mathrm{C}(32)-\mathrm{C}(33)-\mathrm{H}(33) & 119.3 \\ \mathrm{C}(29)-\mathrm{C}(30)-\mathrm{H}(30) & 119.8 & \mathrm{C}(28)-\mathrm{C}(33)-\mathrm{H}(33) & 119.3 \\ \mathrm{C}(30)-\mathrm{C}(31)-\mathrm{C}(32) & 119.0(5) & & \\ \mathrm{C}(30)-\mathrm{C}(31)-\mathrm{H}(31) & 120.5 & \text { Symmetry transformations used to generate } \\ \mathrm{C}(32)-\mathrm{C}(31)-\mathrm{H}(31) & 120.5 & & \\ \mathrm{C}(33)-\mathrm{C}(32)-\mathrm{C}(31) & 120.5(5) & & \end{array}$


Table 4. Anisotropic displacement parameters $\left(\AA^{2} \times 10^{3}\right)$ for 2 . The anisotropic displacement factor exponent takes the form: $-2 \pi^{2}\left[h^{2} a^{* 2} U^{11}+\ldots+2 h k a^{*} b^{*} U^{12}\right]$

\begin{tabular}{|c|c|c|c|c|c|c|}
\hline & $\mathrm{U}^{11}$ & $\mathrm{U}^{22}$ & $\mathrm{U}^{33}$ & $\mathrm{U}^{23}$ & $\mathrm{U}^{13}$ & $\mathrm{U}^{12}$ \\
\hline $\mathrm{Rh}$ & $44(1)$ & $50(1)$ & $18(1)$ & $2(1)$ & $6(1)$ & $-2(1)$ \\
\hline $\mathrm{N}(1)$ & $67(3)$ & $56(3)$ & $23(2)$ & $-2(2)$ & $4(2)$ & $0(2)$ \\
\hline $\mathrm{N}(2)$ & $52(2)$ & $52(2)$ & $26(2)$ & $4(2)$ & $1(2)$ & $-2(2)$ \\
\hline $\mathrm{N}(3)$ & $51(2)$ & $71(3)$ & $26(2)$ & $3(2)$ & $8(2)$ & $2(2)$ \\
\hline $\mathrm{C}(1)$ & $82(4)$ & $70(4)$ & $46(3)$ & $-1(3)$ & $-21(3)$ & $-7(3)$ \\
\hline $\mathrm{C}(2)$ & $68(4)$ & $62(3)$ & $48(3)$ & $5(3)$ & $-16(3)$ & $-14(3)$ \\
\hline $\mathrm{C}(3)$ & $68(4)$ & $62(3)$ & $37(3)$ & $15(2)$ & $5(2)$ & $-4(3)$ \\
\hline $\mathrm{C}(4)$ & $73(4)$ & $109(5)$ & $39(3)$ & $18(3)$ & $15(3)$ & $-13(4)$ \\
\hline $\mathrm{C}(5)$ & $66(4)$ & $98(5)$ & $47(3)$ & $-20(3)$ & $15(3)$ & $18(3)$ \\
\hline $\mathrm{C}(6)$ & $97(5)$ & $58(3)$ & $48(3)$ & $-7(3)$ & $30(3)$ & $-4(3)$ \\
\hline$C(7)$ & $65(3)$ & $54(3)$ & $38(2)$ & $1(2)$ & $4(2)$ & $-10(3)$ \\
\hline $\mathrm{C}(8)$ & $78(4)$ & $77(4)$ & $42(3)$ & $10(3)$ & $1(3)$ & $22(3)$ \\
\hline $\mathrm{C}(9)$ & $53(3)$ & $81(4)$ & $43(3)$ & $3(3)$ & $17(2)$ & $-8(3)$ \\
\hline$C(10)$ & $40(2)$ & $47(3)$ & $25(2)$ & $1(2)$ & $1(2)$ & $-3(2)$ \\
\hline $\mathrm{C}(11)$ & $50(3)$ & $55(3)$ & $32(2)$ & $1(2)$ & $3(2)$ & $-5(2)$ \\
\hline $\mathrm{C}(12)$ & $42(3)$ & $54(3)$ & $45(3)$ & $-11(2)$ & $2(2)$ & $-4(2)$ \\
\hline $\mathrm{C}(13)$ & $57(3)$ & $62(3)$ & $56(3)$ & $-2(3)$ & $10(2)$ & $-10(3)$ \\
\hline$C(14)$ & $80(5)$ & $90(5)$ & $88(5)$ & $-24(4)$ & $29(4)$ & $-37(4)$ \\
\hline $\mathrm{C}(15)$ & $61(4)$ & $86(6)$ & $168(9)$ & $-63(6)$ & $34(5)$ & $-16(4)$ \\
\hline$C(16)$ & $61(4)$ & $69(5)$ & 198(10) & $-38(6)$ & $16(5)$ & $17(4)$ \\
\hline $\mathrm{C}(17)$ & $56(4)$ & $54(4)$ & $128(6)$ & $2(4)$ & $-6(4)$ & $5(3)$ \\
\hline$C(18)$ & $40(2)$ & $56(3)$ & $25(2)$ & $14(2)$ & $-1(2)$ & $-13(2)$ \\
\hline $\mathrm{C}(19)$ & $60(3)$ & $74(4)$ & $25(2)$ & $8(2)$ & $5(2)$ & $5(3)$ \\
\hline $\mathrm{C}(20)$ & $60(3)$ & $62(3)$ & $28(2)$ & $5(2)$ & $7(2)$ & $-5(3)$ \\
\hline$C(21)$ & $64(3)$ & $66(4)$ & $39(3)$ & $4(2)$ & $10(2)$ & $10(3)$ \\
\hline$C(22)$ & $107(5)$ & $65(4)$ & $51(3)$ & $7(3)$ & $-2(3)$ & $2(4)$ \\
\hline$C(23)$ & $116(6)$ & $66(4)$ & $47(3)$ & $8(3)$ & $-2(3)$ & $-20(4)$ \\
\hline$C(24)$ & $73(4)$ & $76(4)$ & $41(3)$ & $14(3)$ & $-5(3)$ & $-20(3)$ \\
\hline$C(25)$ & $63(3)$ & $79(4)$ & $35(3)$ & $7(3)$ & $5(2)$ & $-7(3)$ \\
\hline$C(26)$ & $41(2)$ & $53(3)$ & 21(2) & $-5(2)$ & $7(2)$ & $-5(2)$ \\
\hline$C(27)$ & $42(2)$ & $49(3)$ & $27(2)$ & $-5(2)$ & $6(2)$ & $-5(2)$ \\
\hline
\end{tabular}




\begin{tabular}{lllllll}
$\mathrm{C}(28)$ & $35(2)$ & $53(3)$ & $33(2)$ & $-2(2)$ & $-2(2)$ & $-4(2)$ \\
$\mathrm{C}(29)$ & $53(3)$ & $69(3)$ & $34(2)$ & $3(2)$ & $7(2)$ & $14(3)$ \\
$\mathrm{C}(30)$ & $53(3)$ & $93(4)$ & $33(2)$ & $9(3)$ & $5(2)$ & $14(3)$ \\
$\mathrm{C}(31)$ & $63(3)$ & $66(3)$ & $54(3)$ & $7(3)$ & $-13(3)$ & $12(3)$ \\
$\mathrm{C}(32)$ & $95(5)$ & $75(4)$ & $50(3)$ & $-14(3)$ & $-9(3)$ & $31(3)$ \\
$\mathrm{C}(33)$ & $85(4)$ & $68(4)$ & $31(2)$ & $-8(2)$ & $-2(2)$ & $20(3)$ \\
\hline
\end{tabular}


Table 5. Hydrogen coordinates ( $\left.\mathrm{x} 10^{4}\right)$ and isotropic displacement parameters $\left(\AA^{2} \mathrm{x} 10^{3}\right)$ for 2 .

\begin{tabular}{|c|c|c|c|c|}
\hline & $\mathrm{x}$ & $\mathrm{y}$ & $\mathrm{z}$ & $\mathrm{U}(\mathrm{eq})$ \\
\hline $\mathrm{H}(1 \mathrm{~A})$ & 4108 & 5733 & 7115 & 80 \\
\hline $\mathrm{H}(1 \mathrm{~B})$ & 3633 & 6138 & 8060 & 80 \\
\hline $\mathrm{H}(2 \mathrm{~A})$ & 3593 & 7036 & 6931 & 72 \\
\hline $\mathrm{H}(2 \mathrm{~B})$ & 4831 & 6795 & 6540 & 72 \\
\hline $\mathrm{H}(3 \mathrm{~A})$ & 5617 & 7960 & 6579 & 67 \\
\hline $\mathrm{H}(3 \mathrm{~B})$ & 6154 & 8284 & 7592 & 67 \\
\hline $\mathrm{H}(4 \mathrm{~A})$ & 7617 & 7663 & 6977 & 88 \\
\hline $\mathrm{H}(4 \mathrm{~B})$ & 6801 & 6980 & 6610 & 88 \\
\hline $\mathrm{H}(5 \mathrm{~A})$ & 7993 & 6016 & 7327 & 84 \\
\hline $\mathrm{H}(5 \mathrm{~B})$ & 7766 & 5827 & 8441 & 84 \\
\hline $\mathrm{H}(6 \mathrm{~A})$ & 6454 & 5150 & 7560 & 80 \\
\hline $\mathrm{H}(6 \mathrm{~B})$ & 6109 & 5847 & 6865 & 80 \\
\hline $\mathrm{H}(7 \mathrm{~A})$ & 4818 & 4838 & 8368 & 78 \\
\hline $\mathrm{H}(7 \mathrm{~B})$ & 4482 & 5392 & 9231 & 78 \\
\hline $\mathrm{H}(7 \mathrm{C})$ & 5793 & 5090 & 9176 & 78 \\
\hline $\mathrm{H}(8 \mathrm{~A})$ & 4508 & 8393 & 8476 & 98 \\
\hline $\mathrm{H}(8 \mathrm{~B})$ & 3472 & 7791 & 8365 & 98 \\
\hline $\mathrm{H}(8 \mathrm{C})$ & 3816 & 8284 & 7446 & 98 \\
\hline $\mathrm{H}(9 \mathrm{~A})$ & 8605 & 6897 & 9114 & 88 \\
\hline $\mathrm{H}(9 \mathrm{~B})$ & 8324 & 7722 & 8696 & 88 \\
\hline $\mathrm{H}(9 \mathrm{C})$ & 9038 & 7137 & 8066 & 88 \\
\hline $\mathrm{H}(13)$ & 2637 & 5790 & 11284 & 70 \\
\hline $\mathrm{H}(14)$ & 824 & 5754 & 12002 & 102 \\
\hline $\mathrm{H}(15)$ & -487 & 6715 & 11714 & 125 \\
\hline $\mathrm{H}(16)$ & 3 & 7749 & 10831 & 131 \\
\hline $\mathrm{H}(17)$ & 1814 & 7820 & 10129 & 95 \\
\hline $\mathrm{H}(21)$ & 5757 & 9859 & 9528 & 67 \\
\hline $\mathrm{H}(22)$ & 6418 & 11021 & 10020 & 89 \\
\hline $\mathrm{H}(23)$ & 8233 & 11139 & 10788 & 92 \\
\hline $\mathrm{H}(24)$ & 9429 & 10105 & 11020 & 76 \\
\hline
\end{tabular}




\begin{tabular}{lllll}
$\mathrm{H}(25)$ & 8765 & 8915 & 10531 & 70 \\
$\mathrm{H}(29)$ & 7419 & 5770 & 12540 & 62 \\
$\mathrm{H}(30)$ & 8398 & 4849 & 13459 & 71 \\
$\mathrm{H}(31)$ & 9462 & 3925 & 12700 & 74 \\
$\mathrm{H}(32)$ & 9597 & 3958 & 11007 & 88 \\
$\mathrm{H}(33)$ & 8697 & 4905 & 10098 & 74 \\
\hline
\end{tabular}




\section{X-ray Structure of 3 Important Information}

This molecule had a disordered $\mathrm{BF}_{4}$ molecule that could not be modeled.

Platon's Squeeze routine was used to deal with the disorder. The program accounted for 82 electrons per cell. The number of electrons per cell of the disorder was 82. 


\section{Asymmetric Unit}

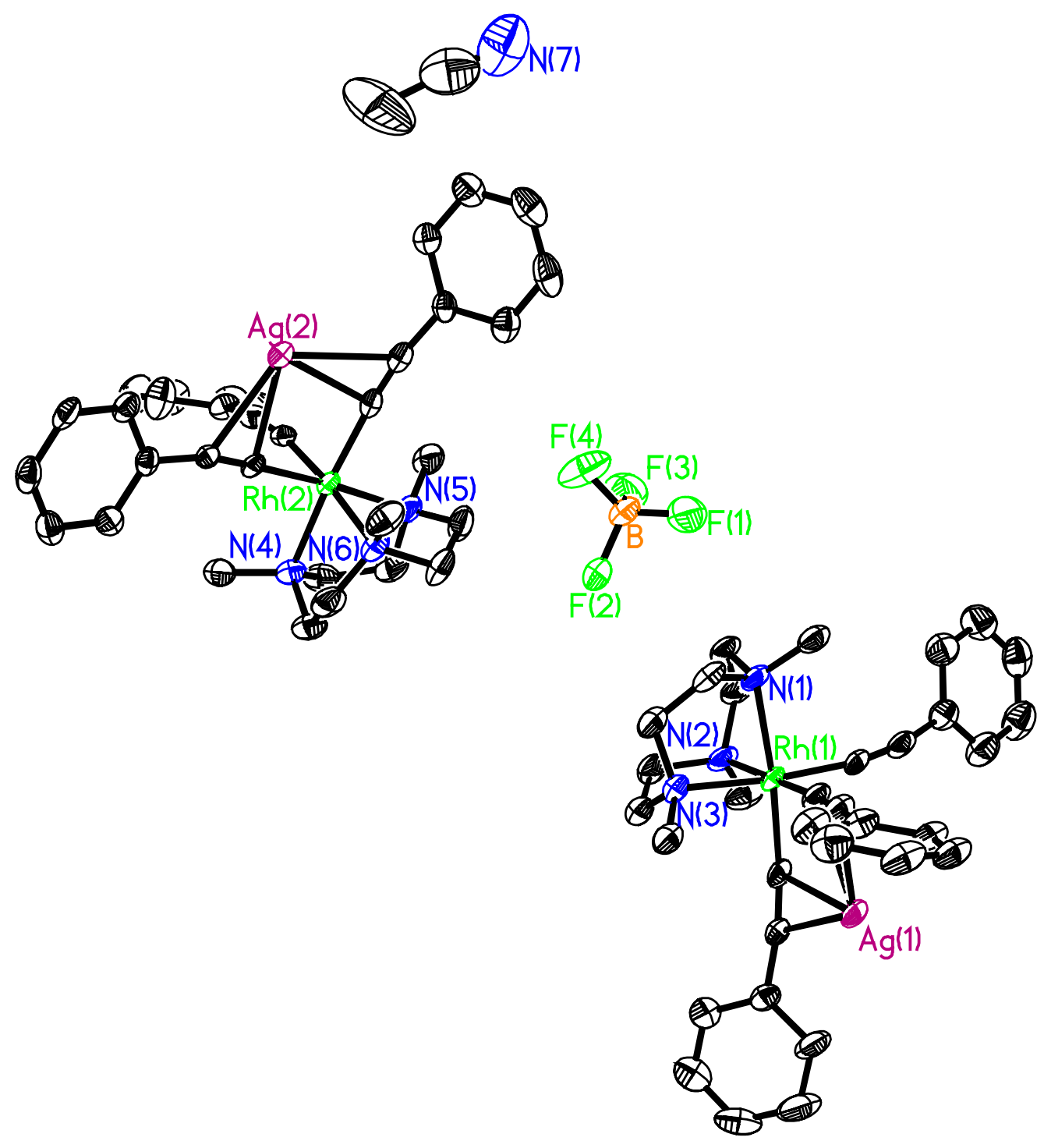




\section{Asym Molecule 1}

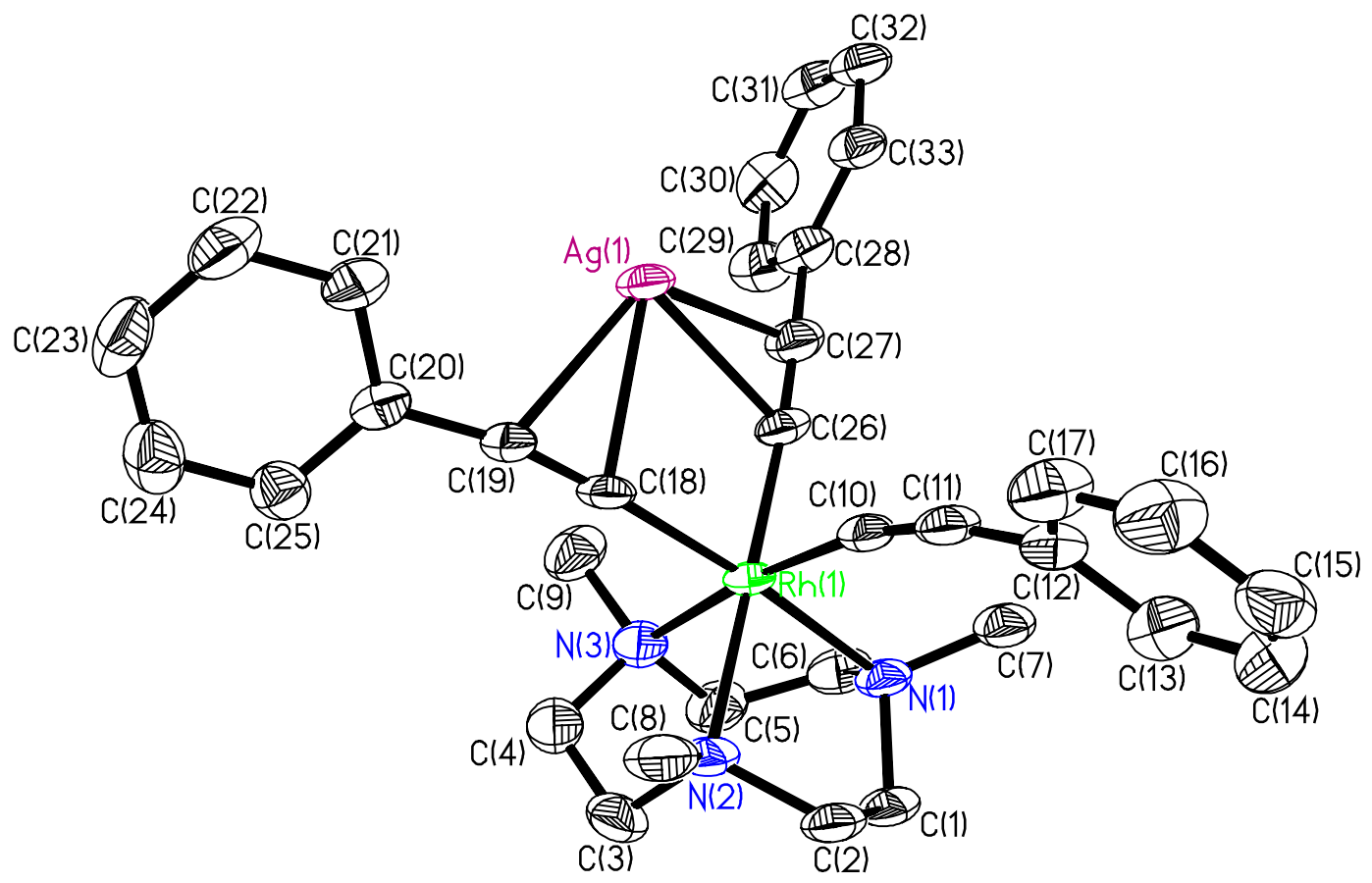




\section{Asym Molecule 2}

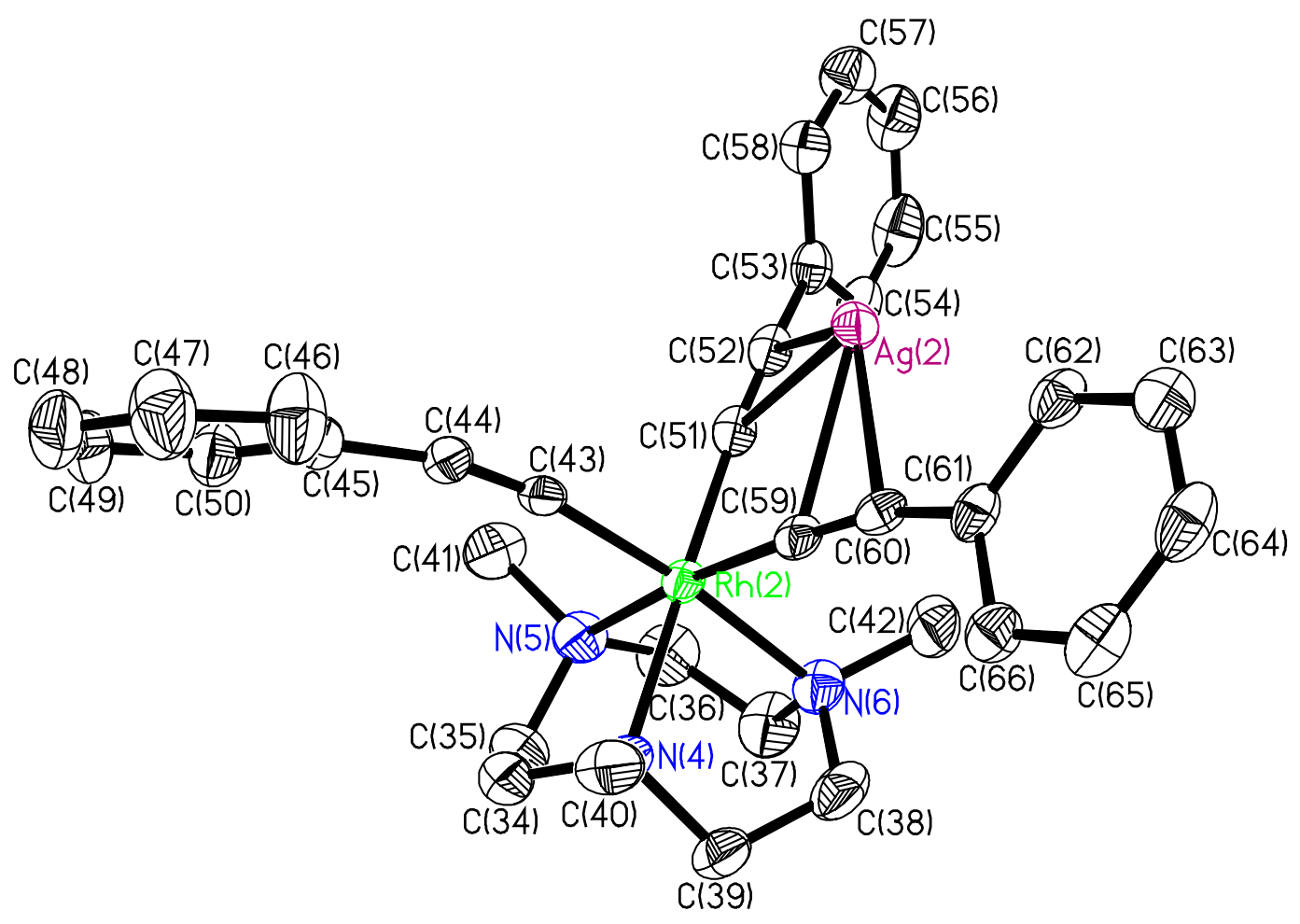




\section{Molecule 1-Ph}

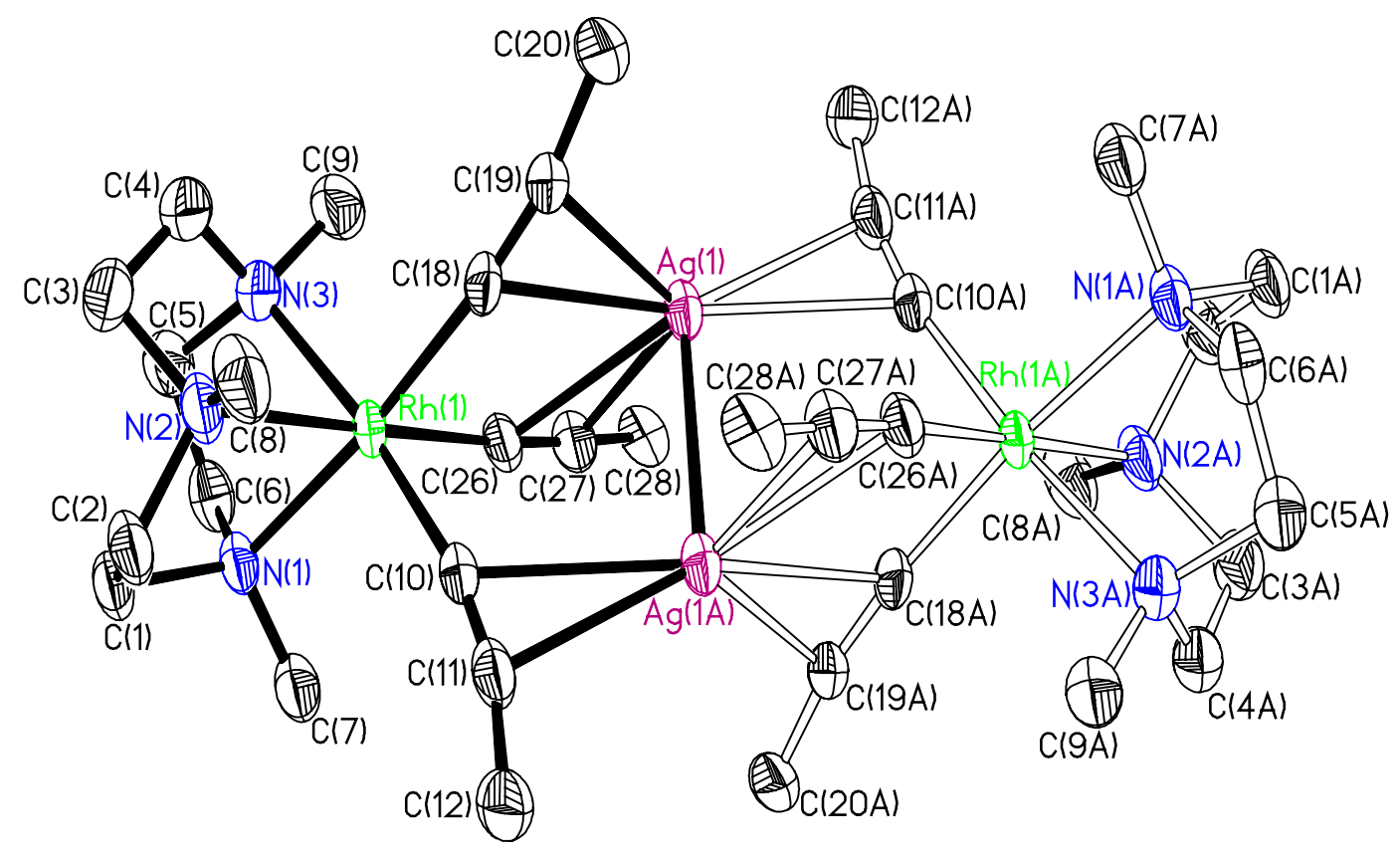

Molecule 2 -Ph

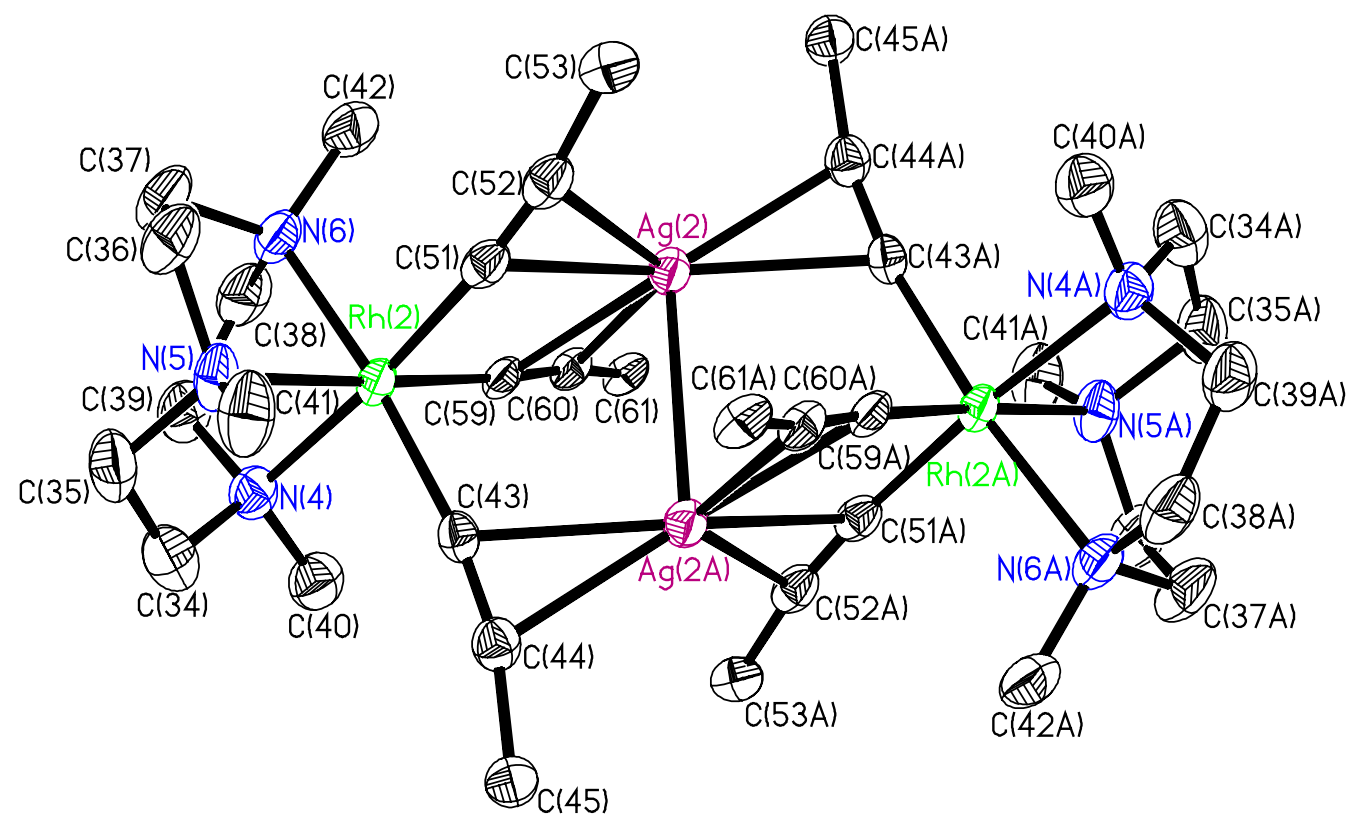




\section{Molecule 1 Axis}

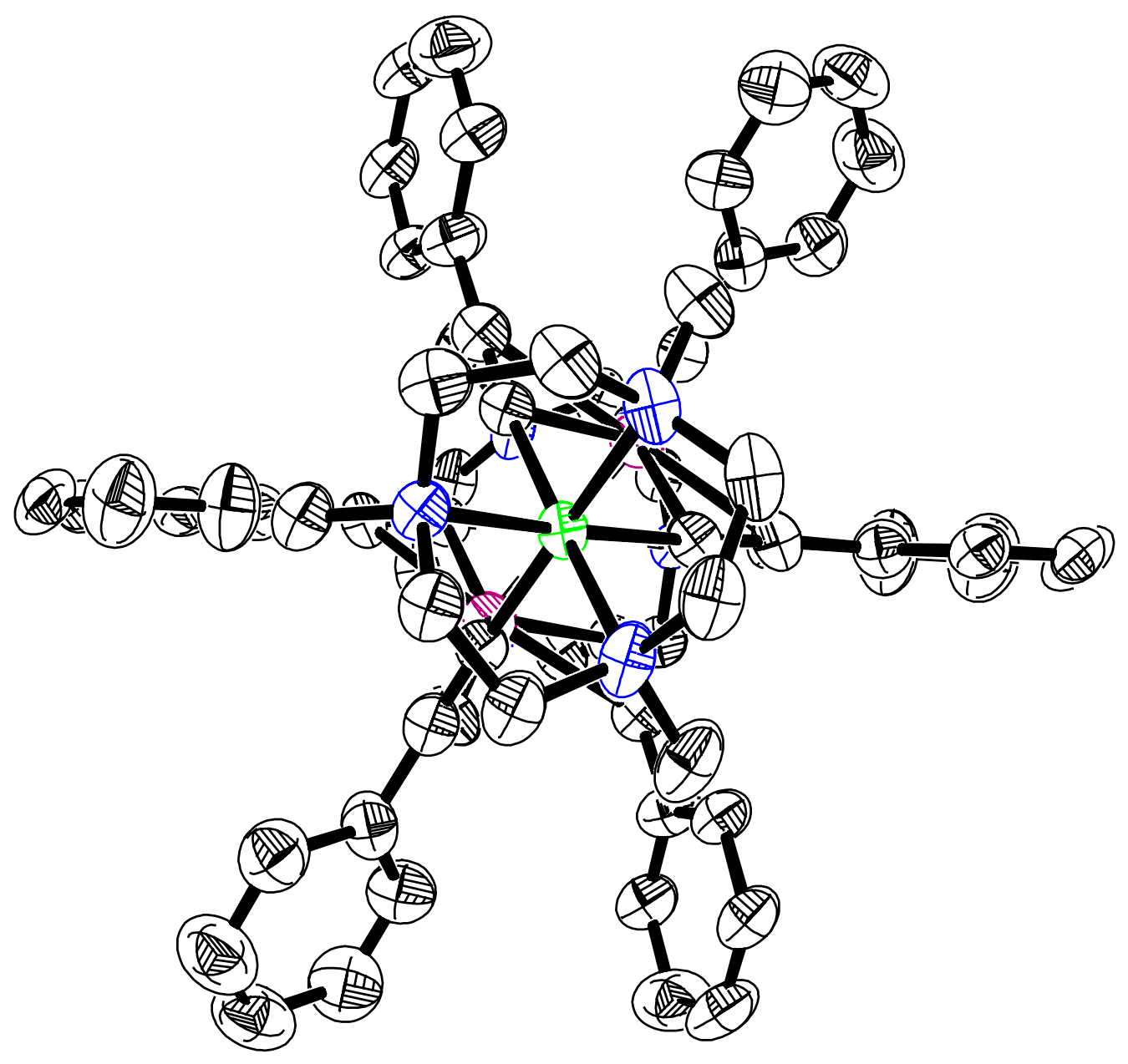




\section{Molecule 2 Axis}

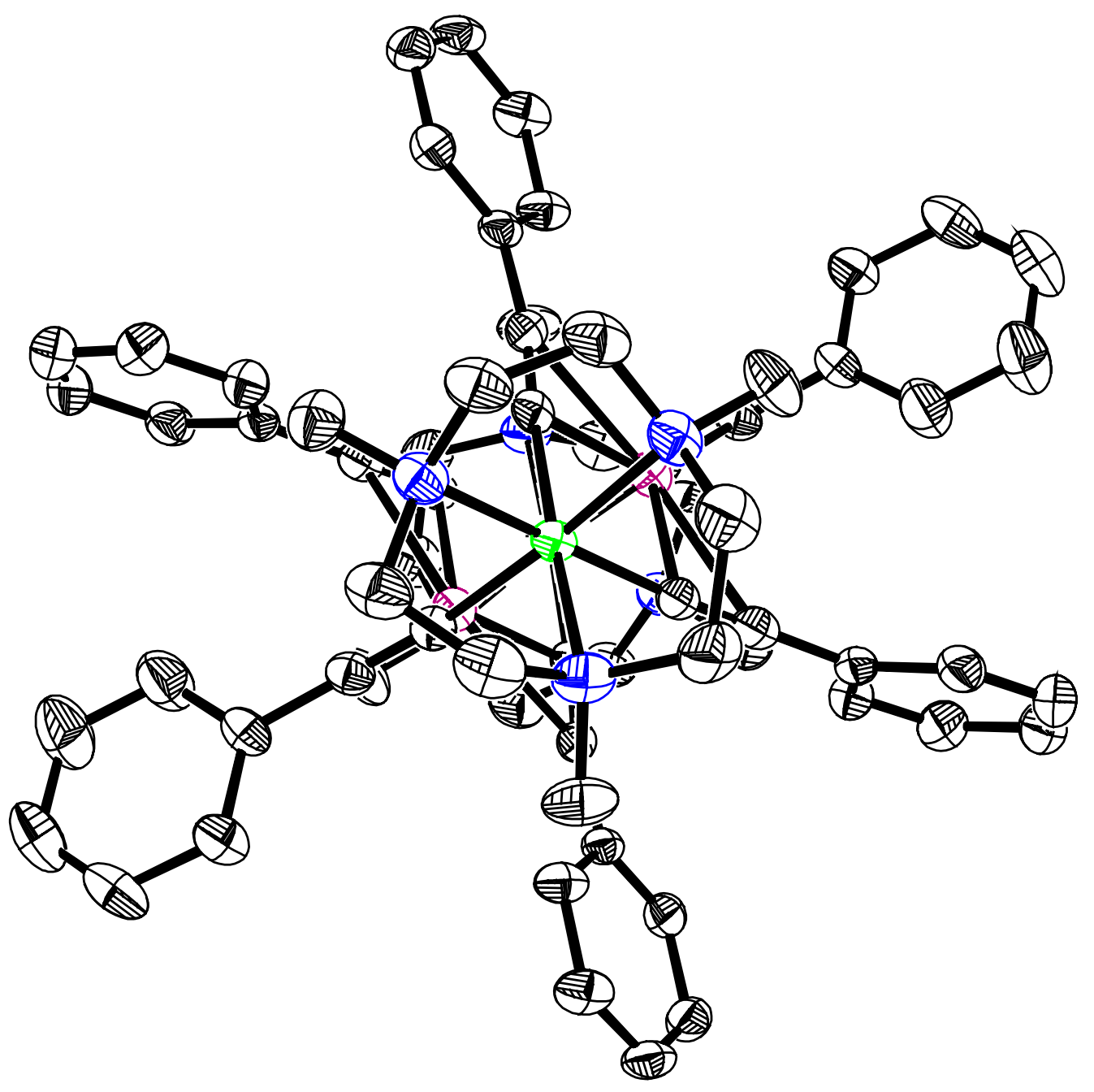




\section{Molecule 1 Side}

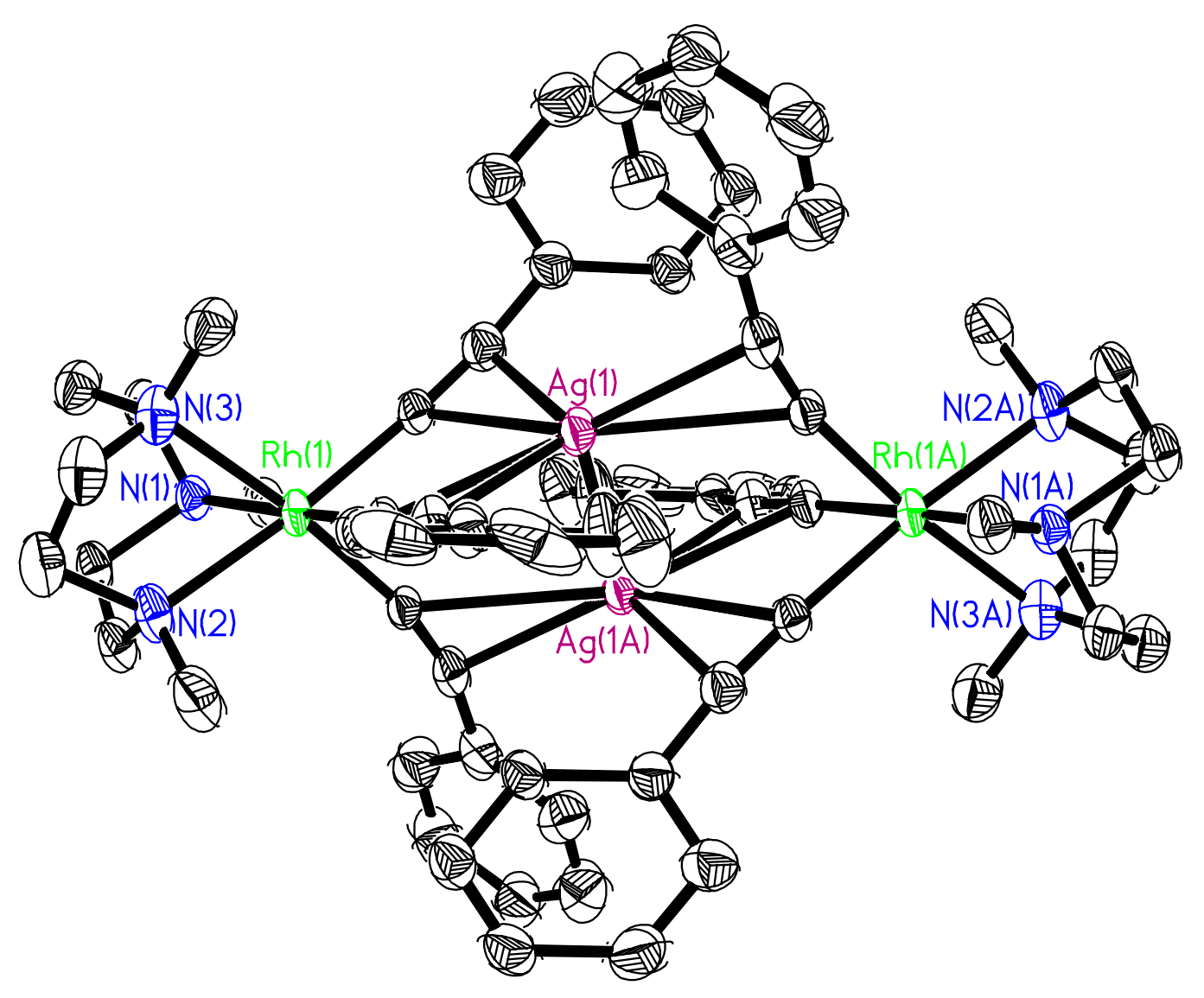




\section{Molecule 2 Side}

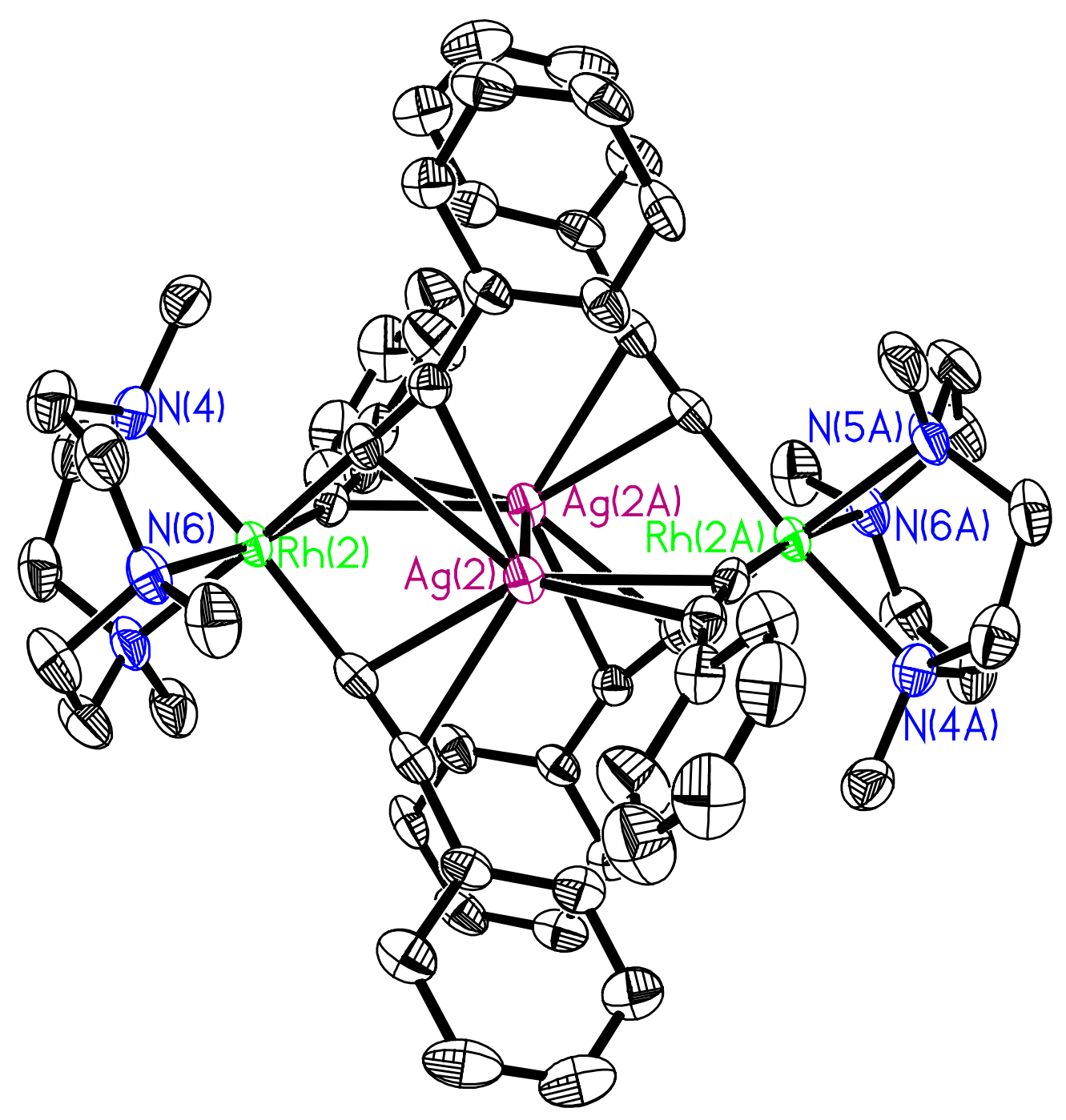


Table 1. Crystal data and structure refinement for 3 .

Identification code

3

Empirical formula

C68 H75 Ag2 B2 F8 N7 Rh2

Formula weight

1585.53

Temperature

100(2) K

Wavelength

$0.71073 \AA$

Crystal system

Triclinic

Space group

Unit cell dimensions

$\mathrm{P}-1$

$\mathrm{a}=13.5520(11) \AA$ $\alpha=77.6600(10)^{\circ}$.

$\mathrm{b}=16.2471(13) \AA$ $\beta=68.1070(10)^{\circ}$.

$c=16.6517(14) \AA$ $\gamma=71.6640(10)^{\circ}$.

Volume

$3209.8(5) \AA^{3}$

$\mathrm{Z}$

2

Density (calculated)

$1.640 \mathrm{Mg} / \mathrm{m}^{3}$

Absorption coefficient

$1.177 \mathrm{~mm}^{-1}$

$\mathrm{F}(000)$

1596

Crystal size

$0.50 \times 0.40 \times 0.10 \mathrm{~mm}^{3}$

Theta range for data collection

1.33 to $27.50^{\circ}$.

Index ranges

$-17 \leq \mathrm{h} \leq 17,-20 \leq \mathrm{k} \leq 20,-21 \leq 1 \leq 21$

Reflections collected

27907

Independent reflections

$14346[\mathrm{R}(\mathrm{int})=0.0297]$

Completeness to theta $=27.50^{\circ}$

Absorption correction

$97.2 \%$

Semi-empirical from equivalents

Max. and min. transmission

0.8914 and 0.5611

Refinement method

Full-matrix least-squares on $\mathrm{F}^{2}$

Data / restraints / parameters

14346 / 0 / 765

Goodness-of-fit on $\mathrm{F}^{2}$

1.076

Final $\mathrm{R}$ indices [I $>2 \operatorname{sigma}(\mathrm{I})]$

$\mathrm{R} 1=0.0543, \mathrm{wR} 2=0.1338$

$\mathrm{R}$ indices (all data)

$\mathrm{R} 1=0.0639, \mathrm{wR} 2=0.1385$

Largest diff. peak and hole

2.670 and -0.732 e. $\AA^{-3}$ 
Table 2. Atomic coordinates ( $x 10^{4}$ ) and equivalent isotropic displacement parameters $\left(\AA^{2} \times 10^{3}\right)$ for 3. $U(e q)$ is defined as one third of the trace of the orthogonalized $U^{i j}$ tensor.

\begin{tabular}{|c|c|c|c|c|}
\hline & $\mathrm{x}$ & $\mathrm{y}$ & $\mathrm{z}$ & $\mathrm{U}(\mathrm{eq})$ \\
\hline $\operatorname{Ag}(1)$ & 8951(1) & $9938(1)$ & $10686(1)$ & $36(1)$ \\
\hline $\operatorname{Ag}(2)$ & $4386(1)$ & $5886(1)$ & 5202(1) & $28(1)$ \\
\hline $\mathrm{Rh}(1)$ & $9534(1)$ & $8645(1)$ & 9101(1) & $31(1)$ \\
\hline $\mathrm{Rh}(2)$ & $6000(1)$ & 4998(1) & $6483(1)$ & $24(1)$ \\
\hline $\mathrm{F}(1)$ & $8646(6)$ & $8569(4)$ & 5991(3) & $113(2)$ \\
\hline $\mathrm{F}(2)$ & $8005(4)$ & $7515(3)$ & 6964(2) & $76(1)$ \\
\hline $\mathrm{F}(3)$ & $9320(4)$ & 7197(4) & 5682(3) & $82(1)$ \\
\hline $\mathrm{F}(4)$ & $7604(4)$ & $7860(3)$ & 5723(3) & $82(1)$ \\
\hline $\mathrm{N}(1)$ & 9362(4) & $8732(3)$ & 7857(3) & $34(1)$ \\
\hline $\mathrm{N}(2)$ & 10386(4) & $7328(3)$ & $8858(3)$ & $42(1)$ \\
\hline $\mathrm{N}(3)$ & $8105(5)$ & $8145(3)$ & 9541(3) & $46(1)$ \\
\hline $\mathrm{N}(4)$ & $5959(4)$ & $4174(3)$ & $7682(3)$ & $34(1)$ \\
\hline $\mathrm{N}(5)$ & $7600(3)$ & $4958(3)$ & 6462(3) & $36(1)$ \\
\hline $\mathrm{N}(6)$ & $5485(4)$ & $6017(3)$ & $7292(3)$ & $35(1)$ \\
\hline $\mathrm{N}(7)$ & $4630(8)$ & $9803(7)$ & $1343(8)$ & $114(3)$ \\
\hline $\mathrm{C}(1)$ & $10010(5)$ & 7890(4) & $7465(4)$ & $41(1)$ \\
\hline$C(2)$ & $10859(5)$ & 7381(4) & $7886(4)$ & $42(1)$ \\
\hline$C(3)$ & $9555(6)$ & $6785(4)$ & 9209(4) & $51(2)$ \\
\hline$C(4)$ & $8519(6)$ & $7232(4)$ & $9867(4)$ & $55(2)$ \\
\hline$C(5)$ & $7734(6)$ & $8212(4)$ & $8779(4)$ & $49(2)$ \\
\hline$C(6)$ & $8146(5)$ & $8879(4)$ & $8065(4)$ & $45(1)$ \\
\hline$C(7)$ & $9678(5)$ & $9476(4)$ & 7234(4) & $42(1)$ \\
\hline $\mathrm{C}(8)$ & 11316(6) & $6935(4)$ & $9221(4)$ & $53(2)$ \\
\hline $\mathrm{C}(9)$ & $7152(6)$ & $8594(5)$ & $10258(4)$ & $53(2)$ \\
\hline $\mathrm{C}(10)$ & 10923(4) & $9014(3)$ & $8592(3)$ & $31(1)$ \\
\hline $\mathrm{C}(11)$ & 11834(5) & $9129(3)$ & 8189(3) & $39(1)$ \\
\hline $\mathrm{C}(12)$ & $12959(5)$ & $9120(3)$ & 7601(4) & $40(1)$ \\
\hline $\mathrm{C}(13)$ & $13200(6)$ & $9095(4)$ & $6710(4)$ & $53(2)$ \\
\hline $\mathrm{C}(14)$ & $14270(6)$ & $9047(5)$ & $6150(5)$ & $60(2)$ \\
\hline$C(15)$ & $15085(6)$ & $9046(4)$ & 6449(5) & $56(2)$ \\
\hline$C(16)$ & 14851(6) & $9070(5)$ & $7327(5)$ & $62(2)$ \\
\hline
\end{tabular}




\begin{tabular}{|c|c|c|c|c|}
\hline $\mathrm{C}(17)$ & 13797(6) & $9108(5)$ & 7895(5) & $54(2)$ \\
\hline $\mathrm{C}(18)$ & $9593(5)$ & $8484(3)$ & 10298(3) & $35(1)$ \\
\hline $\mathrm{C}(19)$ & $9549(5)$ & $8349(3)$ & $11059(3)$ & $37(1)$ \\
\hline $\mathrm{C}(20)$ & $9530(5)$ & 8021(4) & 11944(3) & $36(1)$ \\
\hline $\mathrm{C}(21)$ & $9741(8)$ & $8488(5)$ & $12437(4)$ & $66(2)$ \\
\hline$C(22)$ & $9693(8)$ & $8162(5)$ & $13292(5)$ & $73(2)$ \\
\hline $\mathrm{C}(23)$ & $9424(6)$ & $7379(5)$ & $13649(4)$ & $58(2)$ \\
\hline $\mathrm{C}(24)$ & $9223(5)$ & 6922(4) & 13154(4) & $51(2)$ \\
\hline$C(25)$ & $9275(5)$ & $7232(4)$ & $12310(4)$ & $40(1)$ \\
\hline$C(26)$ & $8622(4)$ & $9865(3)$ & $9316(3)$ & $33(1)$ \\
\hline $\mathrm{C}(27)$ & $8001(5)$ & $10580(4)$ & 9489(4) & $41(1)$ \\
\hline $\mathrm{C}(28)$ & $7182(5)$ & 11397(4) & $9689(4)$ & $42(1)$ \\
\hline$C(29)$ & $6067(6)$ & $11445(4)$ & 9901(4) & $51(2)$ \\
\hline $\mathrm{C}(30)$ & $5268(6)$ & $12234(5)$ & 10131(4) & $54(2)$ \\
\hline $\mathrm{C}(31)$ & $5584(6)$ & 12951(4) & 10137(4) & $51(2)$ \\
\hline $\mathrm{C}(32)$ & $6679(5)$ & $12907(4)$ & 9910(4) & $45(1)$ \\
\hline $\mathrm{C}(33)$ & $7480(5)$ & $12138(4)$ & $9680(3)$ & $38(1)$ \\
\hline $\mathrm{C}(34)$ & $7125(5)$ & $3706(4)$ & $7555(4)$ & $45(1)$ \\
\hline $\mathrm{C}(35)$ & $7846(5)$ & $4338(4)$ & $7209(4)$ & $46(1)$ \\
\hline$C(36)$ & $7499(5)$ & $5864(4)$ & $6585(4)$ & $47(1)$ \\
\hline $\mathrm{C}(37)$ & $6492(5)$ & 6208(4) & 7317(4) & $49(2)$ \\
\hline $\mathrm{C}(38)$ & $4864(5)$ & $5632(4)$ & $8139(4)$ & $45(1)$ \\
\hline $\mathrm{C}(39)$ & $5485(5)$ & $4740(4)$ & 8411(4) & $43(1)$ \\
\hline $\mathrm{C}(40)$ & $5297(5)$ & $3529(4)$ & 7887(4) & $43(1)$ \\
\hline $\mathrm{C}(41)$ & $8500(4)$ & $4718(5)$ & $5637(4)$ & $48(2)$ \\
\hline $\mathrm{C}(42)$ & $4748(5)$ & $6836(4)$ & 7037(4) & $48(2)$ \\
\hline $\mathrm{C}(43)$ & 6501(4) & 3953(3) & $5843(3)$ & $26(1)$ \\
\hline $\mathrm{C}(44)$ & $6798(4)$ & $3244(3)$ & $5578(3)$ & $29(1)$ \\
\hline $\mathrm{C}(45)$ & $7288(4)$ & 2346(3) & 5396(3) & $33(1)$ \\
\hline$C(46)$ & $6642(6)$ & 1791(4) & $5476(5)$ & $57(2)$ \\
\hline $\mathrm{C}(47)$ & 7120(7) & $919(5)$ & $5383(6)$ & $65(2)$ \\
\hline $\mathrm{C}(48)$ & $8236(6)$ & $572(4)$ & $5177(5)$ & $59(2)$ \\
\hline $\mathrm{C}(49)$ & $8864(5)$ & 1110(4) & $5067(5)$ & $55(2)$ \\
\hline $\mathrm{C}(50)$ & $8413(5)$ & 1996(4) & $5180(4)$ & $42(1)$ \\
\hline $\mathrm{C}(51)$ & $6103(4)$ & $5815(3)$ & 5396(3) & $27(1)$ \\
\hline$C(52)$ & $6149(4)$ & 6359(3) & 4782(3) & $30(1)$ \\
\hline
\end{tabular}




\begin{tabular}{lrrrr}
$\mathrm{C}(53)$ & $6287(4)$ & $7074(3)$ & $4086(3)$ & $29(1)$ \\
$\mathrm{C}(54)$ & $6497(4)$ & $7815(4)$ & $4216(4)$ & $39(1)$ \\
$\mathrm{C}(55)$ & $6583(5)$ & $8506(4)$ & $3556(5)$ & $52(2)$ \\
$\mathrm{C}(56)$ & $6461(6)$ & $8470(4)$ & $2775(4)$ & $53(2)$ \\
$\mathrm{C}(57)$ & $6273(5)$ & $7738(4)$ & $2642(4)$ & $45(1)$ \\
$\mathrm{C}(58)$ & $6187(4)$ & $7044(3)$ & $3283(3)$ & $36(1)$ \\
$\mathrm{C}(59)$ & $4460(4)$ & $5090(3)$ & $6595(3)$ & $25(1)$ \\
$\mathrm{C}(60)$ & $3486(4)$ & $5197(3)$ & $6692(3)$ & $25(1)$ \\
$\mathrm{C}(61)$ & $2343(4)$ & $5199(3)$ & $7007(3)$ & $27(1)$ \\
$\mathrm{C}(62)$ & $1677(4)$ & $5585(4)$ & $6486(3)$ & $33(1)$ \\
$\mathrm{C}(63)$ & $562(4)$ & $5616(4)$ & $6818(4)$ & $37(1)$ \\
$\mathrm{C}(64)$ & $110(4)$ & $5256(4)$ & $7659(4)$ & $40(1)$ \\
$\mathrm{C}(65)$ & $763(5)$ & $4861(4)$ & $8174(4)$ & $39(1)$ \\
$\mathrm{C}(66)$ & $1875(4)$ & $4839(3)$ & $7854(3)$ & $33(1)$ \\
$\mathrm{C}(67)$ & $4086(10)$ & $9375(8)$ & $1804(8)$ & $97(3)$ \\
$\mathrm{C}(68)$ & $3330(11)$ & $8842(9)$ & $2424(8)$ & $146(6)$ \\
$\mathrm{B}$ & $8370(8)$ & $7821(6)$ & $6081(5)$ & $58(2)$ \\
& & & & \\
\hline
\end{tabular}




\begin{tabular}{|c|c|c|c|}
\hline \multirow{2}{*}{\multicolumn{2}{|c|}{ Table 3. Bond lengths $[\AA]$ and angles $\left[{ }^{\circ}\right]$ for 3 . }} & \multirow{2}{*}{$\begin{array}{l}\mathrm{N}(2)-\mathrm{C}(8) \\
\mathrm{N}(2)-\mathrm{C}(3)\end{array}$} & \multirow{2}{*}{$\begin{array}{l}1.503(8) \\
1.517(8)\end{array}$} \\
\hline & & & \\
\hline $\operatorname{Ag}(1)-C(10) \# 1$ & $2.363(4)$ & $\mathrm{N}(3)-\mathrm{C}(4)$ & $1.473(8)$ \\
\hline $\mathrm{Ag}(1)-\mathrm{C}(11) \# 1$ & $2.377(5)$ & $\mathrm{N}(3)-\mathrm{C}(9)$ & $1.502(8)$ \\
\hline $\mathrm{Ag}(1)-\mathrm{C}(18)$ & $2.387(5)$ & $\mathrm{N}(3)-\mathrm{C}(5)$ & $1.504(7)$ \\
\hline $\operatorname{Ag}(1)-C(19)$ & $2.466(5)$ & N(4)-C(34) & $1.478(7)$ \\
\hline $\mathrm{Ag}(1)-\mathrm{C}(26)$ & $2.515(5)$ & $\mathrm{N}(4)-\mathrm{C}(40)$ & $1.494(7)$ \\
\hline $\mathrm{Ag}(1)-\mathrm{C}(27)$ & $2.627(6)$ & N(4)-C(39) & $1.502(7)$ \\
\hline $\operatorname{Ag}(1)-\operatorname{Ag}(1) \# 1$ & $2.9439(8)$ & $\mathrm{N}(5)-\mathrm{C}(41)$ & $1.486(7)$ \\
\hline $\mathrm{Ag}(2)-\mathrm{C}(44) \# 2$ & $2.367(5)$ & $\mathrm{N}(5)-\mathrm{C}(36)$ & $1.486(7)$ \\
\hline $\mathrm{Ag}(2)-\mathrm{C}(43) \# 2$ & $2.390(4)$ & $\mathrm{N}(5)-\mathrm{C}(35)$ & $1.496(7)$ \\
\hline $\operatorname{Ag}(2)-C(59)$ & $2.423(5)$ & $\mathrm{N}(6)-\mathrm{C}(38)$ & $1.470(7)$ \\
\hline $\operatorname{Ag}(2)-C(51)$ & $2.430(5)$ & $\mathrm{N}(6)-\mathrm{C}(42)$ & $1.477(7)$ \\
\hline $\operatorname{Ag}(2)-C(60)$ & $2.513(4)$ & $\mathrm{N}(6)-\mathrm{C}(37)$ & $1.509(7)$ \\
\hline $\mathrm{Ag}(2)-\mathrm{C}(52)$ & $2.542(5)$ & $\mathrm{N}(7)-\mathrm{C}(67)$ & $1.135(14)$ \\
\hline $\operatorname{Ag}(2)-\operatorname{Ag}(2) \# 2$ & $2.9134(7)$ & $\mathrm{C}(1)-\mathrm{C}(2)$ & $1.502(8)$ \\
\hline $\operatorname{Rh}(1)-C(10)$ & $1.981(5)$ & $\mathrm{C}(1)-\mathrm{H}(1 \mathrm{~A})$ & 0.9900 \\
\hline $\mathrm{Rh}(1)-\mathrm{C}(18)$ & $1.983(5)$ & $\mathrm{C}(1)-\mathrm{H}(1 \mathrm{~B})$ & 0.9900 \\
\hline $\operatorname{Rh}(1)-C(26)$ & $2.004(5)$ & $\mathrm{C}(2)-\mathrm{H}(2 \mathrm{~A})$ & 0.9900 \\
\hline $\mathrm{Rh}(1)-\mathrm{N}(2)$ & $2.132(4)$ & $\mathrm{C}(2)-\mathrm{H}(2 \mathrm{~B})$ & 0.9900 \\
\hline $\mathrm{Rh}(1)-\mathrm{N}(1)$ & $2.140(4)$ & $C(3)-C(4)$ & $1.500(10)$ \\
\hline $\operatorname{Rh}(1)-\mathrm{N}(3)$ & $2.150(5)$ & $\mathrm{C}(3)-\mathrm{H}(3 \mathrm{~A})$ & 0.9900 \\
\hline $\operatorname{Rh}(2)-C(59)$ & $1.984(5)$ & $\mathrm{C}(3)-\mathrm{H}(3 \mathrm{~B})$ & 0.9900 \\
\hline $\operatorname{Rh}(2)-C(51)$ & $1.989(5)$ & $\mathrm{C}(4)-\mathrm{H}(4 \mathrm{~A})$ & 0.9900 \\
\hline $\operatorname{Rh}(2)-C(43)$ & $1.996(5)$ & $\mathrm{C}(4)-\mathrm{H}(4 \mathrm{~B})$ & 0.9900 \\
\hline $\mathrm{Rh}(2)-\mathrm{N}(5)$ & $2.137(4)$ & $\mathrm{C}(5)-\mathrm{C}(6)$ & $1.495(9)$ \\
\hline $\mathrm{Rh}(2)-\mathrm{N}(6)$ & $2.138(4)$ & $\mathrm{C}(5)-\mathrm{H}(5 \mathrm{~A})$ & 0.9900 \\
\hline $\mathrm{Rh}(2)-\mathrm{N}(4)$ & $2.146(4)$ & $\mathrm{C}(5)-\mathrm{H}(5 \mathrm{~B})$ & 0.9900 \\
\hline $\mathrm{F}(1)-\mathrm{B}$ & $1.343(8)$ & $\mathrm{C}(6)-\mathrm{H}(6 \mathrm{~A})$ & 0.9900 \\
\hline$F(2)-B$ & $1.399(8)$ & $\mathrm{C}(6)-\mathrm{H}(6 \mathrm{~B})$ & 0.9900 \\
\hline $\mathrm{F}(3)-\mathrm{B}$ & $1.398(11)$ & $\mathrm{C}(7)-\mathrm{H}(7 \mathrm{~A})$ & 0.9800 \\
\hline $\mathrm{F}(4)-\mathrm{B}$ & $1.357(9)$ & $\mathrm{C}(7)-\mathrm{H}(7 \mathrm{~B})$ & 0.9800 \\
\hline $\mathrm{N}(1)-\mathrm{C}(7)$ & $1.477(7)$ & $\mathrm{C}(7)-\mathrm{H}(7 \mathrm{C})$ & 0.9800 \\
\hline $\mathrm{N}(1)-\mathrm{C}(6)$ & $1.502(7)$ & $\mathrm{C}(8)-\mathrm{H}(8 \mathrm{~A})$ & 0.9800 \\
\hline $\mathrm{N}(1)-\mathrm{C}(1)$ & $1.505(7)$ & $\mathrm{C}(8)-\mathrm{H}(8 \mathrm{~B})$ & 0.9800 \\
\hline $\mathrm{N}(2)-\mathrm{C}(2)$ & $1.496(7)$ & $\mathrm{C}(8)-\mathrm{H}(8 \mathrm{C})$ & 0.9800 \\
\hline
\end{tabular}




\begin{tabular}{|c|c|c|c|}
\hline $\mathrm{C}(9)-\mathrm{H}(9 \mathrm{~A})$ & 0.9800 & $\mathrm{C}(29)-\mathrm{H}(29)$ & 0.9500 \\
\hline $\mathrm{C}(9)-\mathrm{H}(9 \mathrm{~B})$ & 0.9800 & $C(30)-C(31)$ & $1.364(9)$ \\
\hline $\mathrm{C}(9)-\mathrm{H}(9 \mathrm{C})$ & 0.9800 & $\mathrm{C}(30)-\mathrm{H}(30)$ & 0.9500 \\
\hline $\mathrm{C}(10)-\mathrm{C}(11)$ & $1.218(8)$ & $\mathrm{C}(31)-\mathrm{C}(32)$ & $1.370(9)$ \\
\hline$C(10)-A g(1) \# 1$ & $2.363(4)$ & $\mathrm{C}(31)-\mathrm{H}(31)$ & 0.9500 \\
\hline $\mathrm{C}(11)-\mathrm{C}(12)$ & $1.471(8)$ & $\mathrm{C}(32)-\mathrm{C}(33)$ & $1.389(8)$ \\
\hline $\mathrm{C}(11)-\operatorname{Ag}(1) \# 1$ & $2.377(5)$ & $\mathrm{C}(32)-\mathrm{H}(32)$ & 0.9500 \\
\hline $\mathrm{C}(12)-\mathrm{C}(17)$ & $1.388(9)$ & $\mathrm{C}(33)-\mathrm{H}(33)$ & 0.9500 \\
\hline $\mathrm{C}(12)-\mathrm{C}(13)$ & $1.401(8)$ & $\mathrm{C}(34)-\mathrm{C}(35)$ & $1.516(9)$ \\
\hline $\mathrm{C}(13)-\mathrm{C}(14)$ & $1.388(9)$ & $\mathrm{C}(34)-\mathrm{H}(34 \mathrm{~A})$ & 0.9900 \\
\hline $\mathrm{C}(13)-\mathrm{H}(13)$ & 0.9500 & $\mathrm{C}(34)-\mathrm{H}(34 \mathrm{~B})$ & 0.9900 \\
\hline$C(14)-C(15)$ & $1.370(10)$ & $\mathrm{C}(35)-\mathrm{H}(35 \mathrm{~A})$ & 0.9900 \\
\hline $\mathrm{C}(14)-\mathrm{H}(14)$ & 0.9500 & $\mathrm{C}(35)-\mathrm{H}(35 \mathrm{~B})$ & 0.9900 \\
\hline$C(15)-C(16)$ & $1.382(10)$ & $\mathrm{C}(36)-\mathrm{C}(37)$ & $1.494(9)$ \\
\hline $\mathrm{C}(15)-\mathrm{H}(15)$ & 0.9500 & $\mathrm{C}(36)-\mathrm{H}(36 \mathrm{~A})$ & 0.9900 \\
\hline$C(16)-C(17)$ & $1.378(9)$ & $\mathrm{C}(36)-\mathrm{H}(36 \mathrm{~B})$ & 0.9900 \\
\hline $\mathrm{C}(16)-\mathrm{H}(16)$ & 0.9500 & $\mathrm{C}(37)-\mathrm{H}(37 \mathrm{~A})$ & 0.9900 \\
\hline $\mathrm{C}(17)-\mathrm{H}(17)$ & 0.9500 & $\mathrm{C}(37)-\mathrm{H}(37 \mathrm{~B})$ & 0.9900 \\
\hline $\mathrm{C}(18)-\mathrm{C}(19)$ & $1.221(7)$ & $\mathrm{C}(38)-\mathrm{C}(39)$ & $1.499(9)$ \\
\hline$C(19)-C(20)$ & $1.449(7)$ & $\mathrm{C}(38)-\mathrm{H}(38 \mathrm{~A})$ & 0.9900 \\
\hline$C(20)-C(21)$ & $1.382(8)$ & $\mathrm{C}(38)-\mathrm{H}(38 \mathrm{~B})$ & 0.9900 \\
\hline$C(20)-C(25)$ & $1.387(8)$ & C(39)-H(39A) & 0.9900 \\
\hline$C(21)-C(22)$ & $1.394(9)$ & $\mathrm{C}(39)-\mathrm{H}(39 \mathrm{~B})$ & 0.9900 \\
\hline $\mathrm{C}(21)-\mathrm{H}(21)$ & 0.9500 & $\mathrm{C}(40)-\mathrm{H}(40 \mathrm{~A})$ & 0.9800 \\
\hline $\mathrm{C}(22)-\mathrm{C}(23)$ & $1.384(11)$ & $\mathrm{C}(40)-\mathrm{H}(40 \mathrm{~B})$ & 0.9800 \\
\hline $\mathrm{C}(22)-\mathrm{H}(22)$ & 0.9500 & $\mathrm{C}(40)-\mathrm{H}(40 \mathrm{C})$ & 0.9800 \\
\hline$C(23)-C(24)$ & $1.363(10)$ & $\mathrm{C}(41)-\mathrm{H}(41 \mathrm{~A})$ & 0.9800 \\
\hline $\mathrm{C}(23)-\mathrm{H}(23)$ & 0.9500 & $\mathrm{C}(41)-\mathrm{H}(41 \mathrm{~B})$ & 0.9800 \\
\hline$C(24)-C(25)$ & $1.371(8)$ & $\mathrm{C}(41)-\mathrm{H}(41 \mathrm{C})$ & 0.9800 \\
\hline $\mathrm{C}(24)-\mathrm{H}(24)$ & 0.9500 & $\mathrm{C}(42)-\mathrm{H}(42 \mathrm{~A})$ & 0.9800 \\
\hline $\mathrm{C}(25)-\mathrm{H}(25)$ & 0.9500 & $\mathrm{C}(42)-\mathrm{H}(42 \mathrm{~B})$ & 0.9800 \\
\hline$C(26)-C(27)$ & $1.219(8)$ & $\mathrm{C}(42)-\mathrm{H}(42 \mathrm{C})$ & 0.9800 \\
\hline $\mathrm{C}(27)-\mathrm{C}(28)$ & $1.446(8)$ & $\mathrm{C}(43)-\mathrm{C}(44)$ & $1.216(7)$ \\
\hline $\mathrm{C}(28)-\mathrm{C}(33)$ & $1.380(8)$ & $\mathrm{C}(43)-\operatorname{Ag}(2) \# 2$ & $2.390(4)$ \\
\hline $\mathrm{C}(28)-\mathrm{C}(29)$ & $1.398(9)$ & $\mathrm{C}(44)-\mathrm{C}(45)$ & $1.446(7)$ \\
\hline $\mathrm{C}(29)-\mathrm{C}(30)$ & $1.410(9)$ & $\mathrm{C}(44)-\mathrm{Ag}(2) \# 2$ & $2.367(5)$ \\
\hline
\end{tabular}




\begin{tabular}{|c|c|c|c|}
\hline$C(45)-C(50)$ & $1.381(7)$ & $\mathrm{C}(66)-\mathrm{H}(66)$ & 0.9500 \\
\hline$C(45)-C(46)$ & $1.399(8)$ & $\mathrm{C}(67)-\mathrm{C}(68)$ & $1.500(17)$ \\
\hline $\mathrm{C}(46)-\mathrm{C}(47)$ & $1.375(9)$ & $\mathrm{C}(68)-\mathrm{H}(68 \mathrm{~A})$ & 0.9800 \\
\hline $\mathrm{C}(46)-\mathrm{H}(46)$ & 0.9500 & $\mathrm{C}(68)-\mathrm{H}(68 \mathrm{~B})$ & 0.9800 \\
\hline $\mathrm{C}(47)-\mathrm{C}(48)$ & $1.373(10)$ & $\mathrm{C}(68)-\mathrm{H}(68 \mathrm{C})$ & 0.9800 \\
\hline $\mathrm{C}(47)-\mathrm{H}(47)$ & 0.9500 & & \\
\hline $\mathrm{C}(48)-\mathrm{C}(49)$ & $1.344(10)$ & $\mathrm{C}(10) \# 1-\mathrm{Ag}(1)-\mathrm{C}(11) \# 1$ & $29.77(18)$ \\
\hline $\mathrm{C}(48)-\mathrm{H}(48)$ & 0.9500 & $\mathrm{C}(10) \# 1-\mathrm{Ag}(1)-\mathrm{C}(18)$ & $148.43(19)$ \\
\hline $\mathrm{C}(49)-\mathrm{C}(50)$ & $1.398(8)$ & $\mathrm{C}(11) \# 1-\mathrm{Ag}(1)-\mathrm{C}(18)$ & $147.83(19)$ \\
\hline $\mathrm{C}(49)-\mathrm{H}(49)$ & 0.9500 & $\mathrm{C}(10) \# 1-\mathrm{Ag}(1)-\mathrm{C}(19)$ & $123.89(18)$ \\
\hline $\mathrm{C}(50)-\mathrm{H}(50)$ & 0.9500 & $\mathrm{C}(11) \# 1-\mathrm{Ag}(1)-\mathrm{C}(19)$ & $119.37(18)$ \\
\hline $\mathrm{C}(51)-\mathrm{C}(52)$ & $1.197(7)$ & $C(18)-A g(1)-C(19)$ & $29.08(17)$ \\
\hline $\mathrm{C}(52)-\mathrm{C}(53)$ & $1.458(7)$ & $\mathrm{C}(10) \# 1-\mathrm{Ag}(1)-\mathrm{C}(26)$ & $138.80(18)$ \\
\hline $\mathrm{C}(53)-\mathrm{C}(54)$ & $1.398(7)$ & $\mathrm{C}(11) \# 1-\mathrm{Ag}(1)-\mathrm{C}(26)$ & $136.62(19)$ \\
\hline $\mathrm{C}(53)-\mathrm{C}(58)$ & $1.405(7)$ & $C(18)-A g(1)-C(26)$ & $68.49(18)$ \\
\hline $\mathrm{C}(54)-\mathrm{C}(55)$ & $1.394(9)$ & $C(19)-A g(1)-C(26)$ & $96.38(17)$ \\
\hline $\mathrm{C}(54)-\mathrm{H}(54)$ & 0.9500 & $\mathrm{C}(10) \# 1-\mathrm{Ag}(1)-\mathrm{C}(27)$ & $115.12(18)$ \\
\hline$C(55)-C(56)$ & $1.386(10)$ & $\mathrm{C}(11) \# 1-\mathrm{Ag}(1)-\mathrm{C}(27)$ & $109.50(19)$ \\
\hline $\mathrm{C}(55)-\mathrm{H}(55)$ & 0.9500 & $C(18)-\operatorname{Ag}(1)-C(27)$ & $94.83(18)$ \\
\hline $\mathrm{C}(56)-\mathrm{C}(57)$ & $1.369(9)$ & $C(19)-\operatorname{Ag}(1)-C(27)$ & $120.89(17)$ \\
\hline $\mathrm{C}(56)-\mathrm{H}(56)$ & 0.9500 & $C(26)-\operatorname{Ag}(1)-C(27)$ & $27.33(17)$ \\
\hline $\mathrm{C}(57)-\mathrm{C}(58)$ & $1.379(8)$ & $\mathrm{C}(10) \# 1-\operatorname{Ag}(1)-\operatorname{Ag}(1) \# 1$ & $90.30(13)$ \\
\hline $\mathrm{C}(57)-\mathrm{H}(57)$ & 0.9500 & $\mathrm{C}(11) \# 1-\operatorname{Ag}(1)-\operatorname{Ag}(1) \# 1$ & $119.82(14)$ \\
\hline C(58)-H(58) & 0.9500 & $C(18)-\operatorname{Ag}(1)-\operatorname{Ag}(1) \# 1$ & $80.85(13)$ \\
\hline $\mathrm{C}(59)-\mathrm{C}(60)$ & $1.227(7)$ & $C(19)-\operatorname{Ag}(1)-\operatorname{Ag}(1) \# 1$ & $95.00(13)$ \\
\hline $\mathrm{C}(60)-\mathrm{C}(61)$ & $1.439(6)$ & $C(26)-\operatorname{Ag}(1)-\operatorname{Ag}(1) \# 1$ & $76.66(12)$ \\
\hline $\mathrm{C}(61)-\mathrm{C}(66)$ & $1.396(7)$ & $C(27)-\operatorname{Ag}(1)-\operatorname{Ag}(1) \# 1$ & $87.43(13)$ \\
\hline $\mathrm{C}(61)-\mathrm{C}(62)$ & $1.398(7)$ & $\mathrm{C}(44) \# 2-\mathrm{Ag}(2)-\mathrm{C}(43) \# 2$ & 29.61(16) \\
\hline $\mathrm{C}(62)-\mathrm{C}(63)$ & $1.391(7)$ & $\mathrm{C}(44) \# 2-\mathrm{Ag}(2)-\mathrm{C}(59)$ & $144.67(16)$ \\
\hline $\mathrm{C}(62)-\mathrm{H}(62)$ & 0.9500 & $\mathrm{C}(43) \# 2-\mathrm{Ag}(2)-\mathrm{C}(59)$ & $140.86(15)$ \\
\hline $\mathrm{C}(63)-\mathrm{C}(64)$ & $1.384(8)$ & $\mathrm{C}(44) \# 2-\mathrm{Ag}(2)-\mathrm{C}(51)$ & $140.12(17)$ \\
\hline $\mathrm{C}(63)-\mathrm{H}(63)$ & 0.9500 & $\mathrm{C}(43) \# 2-\mathrm{Ag}(2)-\mathrm{C}(51)$ & $144.88(16)$ \\
\hline $\mathrm{C}(64)-\mathrm{C}(65)$ & $1.379(9)$ & $C(59)-A g(2)-C(51)$ & $69.53(16)$ \\
\hline $\mathrm{C}(64)-\mathrm{H}(64)$ & 0.9500 & $\mathrm{C}(44) \# 2-\mathrm{Ag}(2)-\mathrm{C}(60)$ & $116.55(16)$ \\
\hline $\mathrm{C}(65)-\mathrm{C}(66)$ & $1.390(7)$ & $\mathrm{C}(43) \# 2-\mathrm{Ag}(2)-\mathrm{C}(60)$ & $115.52(15)$ \\
\hline $\mathrm{C}(65)-\mathrm{H}(65)$ & 0.9500 & $C(59)-A g(2)-C(60)$ & $28.71(15)$ \\
\hline
\end{tabular}




\begin{tabular}{|c|c|c|c|}
\hline$C(51)-A g(2)-C(60)$ & $97.65(16)$ & $\mathrm{N}(5)-\mathrm{Rh}(2)-\mathrm{N}(6)$ & $83.17(17)$ \\
\hline $\mathrm{C}(44) \# 2-\mathrm{Ag}(2)-\mathrm{C}(52)$ & $112.75(17)$ & $\mathrm{C}(59)-\mathrm{Rh}(2)-\mathrm{N}(4)$ & $95.16(18)$ \\
\hline $\mathrm{C}(43) \# 2-\mathrm{Ag}(2)-\mathrm{C}(52)$ & $120.79(16)$ & $\mathrm{C}(51)-\mathrm{Rh}(2)-\mathrm{N}(4)$ & $175.30(17)$ \\
\hline$C(59)-\operatorname{Ag}(2)-C(52)$ & $96.63(16)$ & $\mathrm{C}(43)-\mathrm{Rh}(2)-\mathrm{N}(4)$ & $90.58(17)$ \\
\hline $\mathrm{C}(51)-\mathrm{Ag}(2)-\mathrm{C}(52)$ & $27.74(16)$ & $\mathrm{N}(5)-\mathrm{Rh}(2)-\mathrm{N}(4)$ & $82.67(17)$ \\
\hline$C(60)-\operatorname{Ag}(2)-C(52)$ & $123.68(16)$ & $\mathrm{N}(6)-\mathrm{Rh}(2)-\mathrm{N}(4)$ & $83.09(17)$ \\
\hline $\mathrm{C}(44) \# 2-\operatorname{Ag}(2)-\operatorname{Ag}(2) \# 2$ & $120.30(12)$ & $\mathrm{C}(7)-\mathrm{N}(1)-\mathrm{C}(6)$ & $106.8(4)$ \\
\hline $\mathrm{C}(43) \# 2-\operatorname{Ag}(2)-\operatorname{Ag}(2) \# 2$ & $90.83(11)$ & $\mathrm{C}(7)-\mathrm{N}(1)-\mathrm{C}(1)$ & $109.9(4)$ \\
\hline$C(59)-\operatorname{Ag}(2)-\operatorname{Ag}(2) \# 2$ & $75.80(10)$ & $\mathrm{C}(6)-\mathrm{N}(1)-\mathrm{C}(1)$ & $112.0(4)$ \\
\hline$C(51)-\operatorname{Ag}(2)-\operatorname{Ag}(2) \# 2$ & $79.83(11)$ & $\mathrm{C}(7)-\mathrm{N}(1)-\mathrm{Rh}(1)$ & $115.2(3)$ \\
\hline$C(60)-\operatorname{Ag}(2)-\operatorname{Ag}(2) \# 2$ & $85.91(10)$ & $\mathrm{C}(6)-\mathrm{N}(1)-\mathrm{Rh}(1)$ & $103.6(3)$ \\
\hline$C(52)-\operatorname{Ag}(2)-\operatorname{Ag}(2) \# 2$ & $91.88(11)$ & $\mathrm{C}(1)-\mathrm{N}(1)-\mathrm{Rh}(1)$ & $109.2(3)$ \\
\hline $\mathrm{C}(10)-\mathrm{Rh}(1)-\mathrm{C}(18)$ & $92.3(2)$ & $\mathrm{C}(2)-\mathrm{N}(2)-\mathrm{C}(8)$ & $108.2(5)$ \\
\hline $\mathrm{C}(10)-\mathrm{Rh}(1)-\mathrm{C}(26)$ & $92.5(2)$ & $\mathrm{C}(2)-\mathrm{N}(2)-\mathrm{C}(3)$ & 111.2(4) \\
\hline $\mathrm{C}(18)-\mathrm{Rh}(1)-\mathrm{C}(26)$ & $87.6(2)$ & $\mathrm{C}(8)-\mathrm{N}(2)-\mathrm{C}(3)$ & $110.5(5)$ \\
\hline $\mathrm{C}(10)-\mathrm{Rh}(1)-\mathrm{N}(2)$ & $91.7(2)$ & $\mathrm{C}(2)-\mathrm{N}(2)-\mathrm{Rh}(1)$ & $103.6(3)$ \\
\hline $\mathrm{C}(18)-\mathrm{Rh}(1)-\mathrm{N}(2)$ & $94.69(19)$ & $\mathrm{C}(8)-\mathrm{N}(2)-\mathrm{Rh}(1)$ & $114.8(3)$ \\
\hline $\mathrm{C}(26)-\mathrm{Rh}(1)-\mathrm{N}(2)$ & $175.1(2)$ & $\mathrm{C}(3)-\mathrm{N}(2)-\mathrm{Rh}(1)$ & $108.4(4)$ \\
\hline $\mathrm{C}(10)-\mathrm{Rh}(1)-\mathrm{N}(1)$ & $93.11(18)$ & $\mathrm{C}(4)-\mathrm{N}(3)-\mathrm{C}(9)$ & $109.4(5)$ \\
\hline $\mathrm{C}(18)-\mathrm{Rh}(1)-\mathrm{N}(1)$ & $174.17(19)$ & $\mathrm{C}(4)-\mathrm{N}(3)-\mathrm{C}(5)$ & $112.0(4)$ \\
\hline $\mathrm{C}(26)-\mathrm{Rh}(1)-\mathrm{N}(1)$ & $94.26(18)$ & $\mathrm{C}(9)-\mathrm{N}(3)-\mathrm{C}(5)$ & $108.9(5)$ \\
\hline $\mathrm{N}(2)-\mathrm{Rh}(1)-\mathrm{N}(1)$ & $83.02(16)$ & $\mathrm{C}(4)-\mathrm{N}(3)-\mathrm{Rh}(1)$ & $103.0(4)$ \\
\hline $\mathrm{C}(10)-\mathrm{Rh}(1)-\mathrm{N}(3)$ & $173.49(18)$ & $\mathrm{C}(9)-\mathrm{N}(3)-\mathrm{Rh}(1)$ & $115.0(3)$ \\
\hline $\mathrm{C}(18)-\mathrm{Rh}(1)-\mathrm{N}(3)$ & $92.0(2)$ & $\mathrm{C}(5)-\mathrm{N}(3)-\mathrm{Rh}(1)$ & $108.7(3)$ \\
\hline $\mathrm{C}(26)-\mathrm{Rh}(1)-\mathrm{N}(3)$ & $92.6(2)$ & $\mathrm{C}(34)-\mathrm{N}(4)-\mathrm{C}(40)$ & $109.5(5)$ \\
\hline $\mathrm{N}(2)-\mathrm{Rh}(1)-\mathrm{N}(3)$ & $83.0(2)$ & $\mathrm{C}(34)-\mathrm{N}(4)-\mathrm{C}(39)$ & $112.1(4)$ \\
\hline $\mathrm{N}(1)-\mathrm{Rh}(1)-\mathrm{N}(3)$ & $82.44(17)$ & $\mathrm{C}(40)-\mathrm{N}(4)-\mathrm{C}(39)$ & $108.5(4)$ \\
\hline $\mathrm{C}(59)-\mathrm{Rh}(2)-\mathrm{C}(51)$ & $88.30(19)$ & $\mathrm{C}(34)-\mathrm{N}(4)-\mathrm{Rh}(2)$ & 104.1(3) \\
\hline $\mathrm{C}(59)-\mathrm{Rh}(2)-\mathrm{C}(43)$ & $89.33(18)$ & $\mathrm{C}(40)-\mathrm{N}(4)-\mathrm{Rh}(2)$ & 114.1(3) \\
\hline $\mathrm{C}(51)-\mathrm{Rh}(2)-\mathrm{C}(43)$ & $92.63(18)$ & $\mathrm{C}(39)-\mathrm{N}(4)-\mathrm{Rh}(2)$ & $108.6(3)$ \\
\hline $\mathrm{C}(59)-\mathrm{Rh}(2)-\mathrm{N}(5)$ & $174.90(17)$ & $\mathrm{C}(41)-\mathrm{N}(5)-\mathrm{C}(36)$ & $109.2(5)$ \\
\hline $\mathrm{C}(51)-\mathrm{Rh}(2)-\mathrm{N}(5)$ & 93.61(18) & $\mathrm{C}(41)-\mathrm{N}(5)-\mathrm{C}(35)$ & $109.8(5)$ \\
\hline $\mathrm{C}(43)-\mathrm{Rh}(2)-\mathrm{N}(5)$ & $95.30(18)$ & $\mathrm{C}(36)-\mathrm{N}(5)-\mathrm{C}(35)$ & $110.2(4)$ \\
\hline $\mathrm{C}(59)-\mathrm{Rh}(2)-\mathrm{N}(6)$ & $92.00(17)$ & $\mathrm{C}(41)-\mathrm{N}(5)-\mathrm{Rh}(2)$ & $114.6(3)$ \\
\hline $\mathrm{C}(51)-\mathrm{Rh}(2)-\mathrm{N}(6)$ & $93.64(18)$ & $\mathrm{C}(36)-\mathrm{N}(5)-\mathrm{Rh}(2)$ & $103.9(3)$ \\
\hline $\mathrm{C}(43)-\mathrm{Rh}(2)-\mathrm{N}(6)$ & $173.62(18)$ & $\mathrm{C}(35)-\mathrm{N}(5)-\mathrm{Rh}(2)$ & $109.0(3)$ \\
\hline
\end{tabular}




\begin{tabular}{|c|c|c|c|}
\hline $\mathrm{C}(38)-\mathrm{N}(6)-\mathrm{C}(42)$ & $108.3(5)$ & $\mathrm{C}(5)-\mathrm{C}(6)-\mathrm{N}(1)$ & $110.0(5)$ \\
\hline $\mathrm{C}(38)-\mathrm{N}(6)-\mathrm{C}(37)$ & $112.2(5)$ & $\mathrm{C}(5)-\mathrm{C}(6)-\mathrm{H}(6 \mathrm{~A})$ & 109.7 \\
\hline $\mathrm{C}(42)-\mathrm{N}(6)-\mathrm{C}(37)$ & $109.5(5)$ & $\mathrm{N}(1)-\mathrm{C}(6)-\mathrm{H}(6 \mathrm{~A})$ & 109.7 \\
\hline $\mathrm{C}(38)-\mathrm{N}(6)-\mathrm{Rh}(2)$ & $102.5(3)$ & $\mathrm{C}(5)-\mathrm{C}(6)-\mathrm{H}(6 \mathrm{~B})$ & 109.7 \\
\hline $\mathrm{C}(42)-\mathrm{N}(6)-\mathrm{Rh}(2)$ & $115.4(3)$ & $\mathrm{N}(1)-\mathrm{C}(6)-\mathrm{H}(6 \mathrm{~B})$ & 109.7 \\
\hline $\mathrm{C}(37)-\mathrm{N}(6)-\mathrm{Rh}(2)$ & $108.8(3)$ & $\mathrm{H}(6 \mathrm{~A})-\mathrm{C}(6)-\mathrm{H}(6 \mathrm{~B})$ & 108.2 \\
\hline $\mathrm{C}(2)-\mathrm{C}(1)-\mathrm{N}(1)$ & 111.3(4) & $\mathrm{N}(1)-\mathrm{C}(7)-\mathrm{H}(7 \mathrm{~A})$ & 109.5 \\
\hline $\mathrm{C}(2)-\mathrm{C}(1)-\mathrm{H}(1 \mathrm{~A})$ & 109.4 & $\mathrm{~N}(1)-\mathrm{C}(7)-\mathrm{H}(7 \mathrm{~B})$ & 109.5 \\
\hline $\mathrm{N}(1)-\mathrm{C}(1)-\mathrm{H}(1 \mathrm{~A})$ & 109.4 & $\mathrm{H}(7 \mathrm{~A})-\mathrm{C}(7)-\mathrm{H}(7 \mathrm{~B})$ & 109.5 \\
\hline $\mathrm{C}(2)-\mathrm{C}(1)-\mathrm{H}(1 \mathrm{~B})$ & 109.4 & $\mathrm{~N}(1)-\mathrm{C}(7)-\mathrm{H}(7 \mathrm{C})$ & 109.5 \\
\hline $\mathrm{N}(1)-\mathrm{C}(1)-\mathrm{H}(1 \mathrm{~B})$ & 109.4 & $\mathrm{H}(7 \mathrm{~A})-\mathrm{C}(7)-\mathrm{H}(7 \mathrm{C})$ & 109.5 \\
\hline $\mathrm{H}(1 \mathrm{~A})-\mathrm{C}(1)-\mathrm{H}(1 \mathrm{~B})$ & 108.0 & $\mathrm{H}(7 \mathrm{~B})-\mathrm{C}(7)-\mathrm{H}(7 \mathrm{C})$ & 109.5 \\
\hline $\mathrm{N}(2)-\mathrm{C}(2)-\mathrm{C}(1)$ & $111.7(5)$ & $\mathrm{N}(2)-\mathrm{C}(8)-\mathrm{H}(8 \mathrm{~A})$ & 109.5 \\
\hline $\mathrm{N}(2)-\mathrm{C}(2)-\mathrm{H}(2 \mathrm{~A})$ & 109.3 & $\mathrm{~N}(2)-\mathrm{C}(8)-\mathrm{H}(8 \mathrm{~B})$ & 109.5 \\
\hline $\mathrm{C}(1)-\mathrm{C}(2)-\mathrm{H}(2 \mathrm{~A})$ & 109.3 & $\mathrm{H}(8 \mathrm{~A})-\mathrm{C}(8)-\mathrm{H}(8 \mathrm{~B})$ & 109.5 \\
\hline $\mathrm{N}(2)-\mathrm{C}(2)-\mathrm{H}(2 \mathrm{~B})$ & 109.3 & $\mathrm{~N}(2)-\mathrm{C}(8)-\mathrm{H}(8 \mathrm{C})$ & 109.5 \\
\hline $\mathrm{C}(1)-\mathrm{C}(2)-\mathrm{H}(2 \mathrm{~B})$ & 109.3 & $\mathrm{H}(8 \mathrm{~A})-\mathrm{C}(8)-\mathrm{H}(8 \mathrm{C})$ & 109.5 \\
\hline $\mathrm{H}(2 \mathrm{~A})-\mathrm{C}(2)-\mathrm{H}(2 \mathrm{~B})$ & 107.9 & $\mathrm{H}(8 \mathrm{~B})-\mathrm{C}(8)-\mathrm{H}(8 \mathrm{C})$ & 109.5 \\
\hline $\mathrm{C}(4)-\mathrm{C}(3)-\mathrm{N}(2)$ & $111.5(5)$ & $\mathrm{N}(3)-\mathrm{C}(9)-\mathrm{H}(9 \mathrm{~A})$ & 109.5 \\
\hline $\mathrm{C}(4)-\mathrm{C}(3)-\mathrm{H}(3 \mathrm{~A})$ & 109.3 & $\mathrm{~N}(3)-\mathrm{C}(9)-\mathrm{H}(9 \mathrm{~B})$ & 109.5 \\
\hline $\mathrm{N}(2)-\mathrm{C}(3)-\mathrm{H}(3 \mathrm{~A})$ & 109.3 & $\mathrm{H}(9 \mathrm{~A})-\mathrm{C}(9)-\mathrm{H}(9 \mathrm{~B})$ & 109.5 \\
\hline $\mathrm{C}(4)-\mathrm{C}(3)-\mathrm{H}(3 \mathrm{~B})$ & 109.3 & $\mathrm{~N}(3)-\mathrm{C}(9)-\mathrm{H}(9 \mathrm{C})$ & 109.5 \\
\hline $\mathrm{N}(2)-\mathrm{C}(3)-\mathrm{H}(3 \mathrm{~B})$ & 109.3 & $\mathrm{H}(9 \mathrm{~A})-\mathrm{C}(9)-\mathrm{H}(9 \mathrm{C})$ & 109.5 \\
\hline $\mathrm{H}(3 \mathrm{~A})-\mathrm{C}(3)-\mathrm{H}(3 \mathrm{~B})$ & 108.0 & $\mathrm{H}(9 \mathrm{~B})-\mathrm{C}(9)-\mathrm{H}(9 \mathrm{C})$ & 109.5 \\
\hline $\mathrm{N}(3)-\mathrm{C}(4)-\mathrm{C}(3)$ & $111.4(5)$ & $\mathrm{C}(11)-\mathrm{C}(10)-\mathrm{Rh}(1)$ & $168.9(4)$ \\
\hline $\mathrm{N}(3)-\mathrm{C}(4)-\mathrm{H}(4 \mathrm{~A})$ & 109.3 & $\mathrm{C}(11)-\mathrm{C}(10)-\mathrm{Ag}(1) \# 1$ & $75.8(3)$ \\
\hline $\mathrm{C}(3)-\mathrm{C}(4)-\mathrm{H}(4 \mathrm{~A})$ & 109.3 & $\mathrm{Rh}(1)-\mathrm{C}(10)-\operatorname{Ag}(1) \# 1$ & $115.3(2)$ \\
\hline $\mathrm{N}(3)-\mathrm{C}(4)-\mathrm{H}(4 \mathrm{~B})$ & 109.3 & $\mathrm{C}(10)-\mathrm{C}(11)-\mathrm{C}(12)$ & $168.0(5)$ \\
\hline $\mathrm{C}(3)-\mathrm{C}(4)-\mathrm{H}(4 \mathrm{~B})$ & 109.3 & $\mathrm{C}(10)-\mathrm{C}(11)-\mathrm{Ag}(1) \# 1$ & $74.5(3)$ \\
\hline $\mathrm{H}(4 \mathrm{~A})-\mathrm{C}(4)-\mathrm{H}(4 \mathrm{~B})$ & 108.0 & $\mathrm{C}(12)-\mathrm{C}(11)-\mathrm{Ag}(1) \# 1$ & $117.5(4)$ \\
\hline$C(6)-C(5)-N(3)$ & $111.7(5)$ & $\mathrm{C}(17)-\mathrm{C}(12)-\mathrm{C}(13)$ & $118.8(6)$ \\
\hline $\mathrm{C}(6)-\mathrm{C}(5)-\mathrm{H}(5 \mathrm{~A})$ & 109.3 & $\mathrm{C}(17)-\mathrm{C}(12)-\mathrm{C}(11)$ & $122.7(5)$ \\
\hline $\mathrm{N}(3)-\mathrm{C}(5)-\mathrm{H}(5 \mathrm{~A})$ & 109.3 & $\mathrm{C}(13)-\mathrm{C}(12)-\mathrm{C}(11)$ & $118.5(6)$ \\
\hline $\mathrm{C}(6)-\mathrm{C}(5)-\mathrm{H}(5 \mathrm{~B})$ & 109.3 & $\mathrm{C}(14)-\mathrm{C}(13)-\mathrm{C}(12)$ & 119.3(7) \\
\hline $\mathrm{N}(3)-\mathrm{C}(5)-\mathrm{H}(5 \mathrm{~B})$ & 109.3 & $\mathrm{C}(14)-\mathrm{C}(13)-\mathrm{H}(13)$ & 120.4 \\
\hline $\mathrm{H}(5 \mathrm{~A})-\mathrm{C}(5)-\mathrm{H}(5 \mathrm{~B})$ & 107.9 & $\mathrm{C}(12)-\mathrm{C}(13)-\mathrm{H}(13)$ & 120.4 \\
\hline
\end{tabular}




\begin{tabular}{|c|c|c|c|}
\hline$C(15)-C(14)-C(13)$ & $121.2(7)$ & $C(27)-C(26)-R h(1)$ & $175.0(5)$ \\
\hline $\mathrm{C}(15)-\mathrm{C}(14)-\mathrm{H}(14)$ & 119.4 & $\mathrm{C}(27)-\mathrm{C}(26)-\mathrm{Ag}(1)$ & $81.4(3)$ \\
\hline $\mathrm{C}(13)-\mathrm{C}(14)-\mathrm{H}(14)$ & 119.4 & $\mathrm{Rh}(1)-\mathrm{C}(26)-\mathrm{Ag}(1)$ & $98.2(2)$ \\
\hline$C(14)-C(15)-C(16)$ & $119.7(6)$ & $\mathrm{C}(26)-\mathrm{C}(27)-\mathrm{C}(28)$ & $174.4(6)$ \\
\hline $\mathrm{C}(14)-\mathrm{C}(15)-\mathrm{H}(15)$ & 120.2 & $\mathrm{C}(26)-\mathrm{C}(27)-\mathrm{Ag}(1)$ & $71.2(4)$ \\
\hline $\mathrm{C}(16)-\mathrm{C}(15)-\mathrm{H}(15)$ & 120.2 & $\mathrm{C}(28)-\mathrm{C}(27)-\operatorname{Ag}(1)$ & $112.0(3)$ \\
\hline $\mathrm{C}(17)-\mathrm{C}(16)-\mathrm{C}(15)$ & $119.9(7)$ & $\mathrm{C}(33)-\mathrm{C}(28)-\mathrm{C}(29)$ & $118.9(6)$ \\
\hline $\mathrm{C}(17)-\mathrm{C}(16)-\mathrm{H}(16)$ & 120.0 & $\mathrm{C}(33)-\mathrm{C}(28)-\mathrm{C}(27)$ & $121.1(5)$ \\
\hline $\mathrm{C}(15)-\mathrm{C}(16)-\mathrm{H}(16)$ & 120.0 & $\mathrm{C}(29)-\mathrm{C}(28)-\mathrm{C}(27)$ & $120.0(5)$ \\
\hline$C(16)-C(17)-C(12)$ & $121.0(7)$ & $\mathrm{C}(28)-\mathrm{C}(29)-\mathrm{C}(30)$ & $119.8(6)$ \\
\hline $\mathrm{C}(16)-\mathrm{C}(17)-\mathrm{H}(17)$ & 119.5 & $\mathrm{C}(28)-\mathrm{C}(29)-\mathrm{H}(29)$ & 120.1 \\
\hline $\mathrm{C}(12)-\mathrm{C}(17)-\mathrm{H}(17)$ & 119.5 & $\mathrm{C}(30)-\mathrm{C}(29)-\mathrm{H}(29)$ & 120.1 \\
\hline $\mathrm{C}(19)-\mathrm{C}(18)-\mathrm{Rh}(1)$ & $174.0(5)$ & $C(31)-C(30)-C(29)$ & $120.1(6)$ \\
\hline $\mathrm{C}(19)-\mathrm{C}(18)-\mathrm{Ag}(1)$ & 79.1(3) & $\mathrm{C}(31)-\mathrm{C}(30)-\mathrm{H}(30)$ & 120.0 \\
\hline $\mathrm{Rh}(1)-\mathrm{C}(18)-\mathrm{Ag}(1)$ & $103.1(2)$ & $\mathrm{C}(29)-\mathrm{C}(30)-\mathrm{H}(30)$ & 120.0 \\
\hline$C(18)-C(19)-C(20)$ & $169.3(5)$ & $C(30)-C(31)-C(32)$ & $120.0(6)$ \\
\hline$C(18)-C(19)-\operatorname{Ag}(1)$ & $71.9(3)$ & $\mathrm{C}(30)-\mathrm{C}(31)-\mathrm{H}(31)$ & 120.0 \\
\hline$C(20)-C(19)-\operatorname{Ag}(1)$ & $118.6(3)$ & $\mathrm{C}(32)-\mathrm{C}(31)-\mathrm{H}(31)$ & 120.0 \\
\hline $\mathrm{C}(21)-\mathrm{C}(20)-\mathrm{C}(25)$ & $119.3(5)$ & $\mathrm{C}(31)-\mathrm{C}(32)-\mathrm{C}(33)$ & $121.0(6)$ \\
\hline$C(21)-C(20)-C(19)$ & $121.2(5)$ & $\mathrm{C}(31)-\mathrm{C}(32)-\mathrm{H}(32)$ & 119.5 \\
\hline $\mathrm{C}(25)-\mathrm{C}(20)-\mathrm{C}(19)$ & $119.5(5)$ & $\mathrm{C}(33)-\mathrm{C}(32)-\mathrm{H}(32)$ & 119.5 \\
\hline $\mathrm{C}(20)-\mathrm{C}(21)-\mathrm{C}(22)$ & 119.7(7) & $\mathrm{C}(28)-\mathrm{C}(33)-\mathrm{C}(32)$ & $120.3(6)$ \\
\hline $\mathrm{C}(20)-\mathrm{C}(21)-\mathrm{H}(21)$ & 120.1 & $\mathrm{C}(28)-\mathrm{C}(33)-\mathrm{H}(33)$ & 119.9 \\
\hline $\mathrm{C}(22)-\mathrm{C}(21)-\mathrm{H}(21)$ & 120.1 & $\mathrm{C}(32)-\mathrm{C}(33)-\mathrm{H}(33)$ & 119.9 \\
\hline $\mathrm{C}(23)-\mathrm{C}(22)-\mathrm{C}(21)$ & $120.1(6)$ & $\mathrm{N}(4)-\mathrm{C}(34)-\mathrm{C}(35)$ & $110.7(5)$ \\
\hline $\mathrm{C}(23)-\mathrm{C}(22)-\mathrm{H}(22)$ & 119.9 & $\mathrm{~N}(4)-\mathrm{C}(34)-\mathrm{H}(34 \mathrm{~A})$ & 109.5 \\
\hline $\mathrm{C}(21)-\mathrm{C}(22)-\mathrm{H}(22)$ & 119.9 & $\mathrm{C}(35)-\mathrm{C}(34)-\mathrm{H}(34 \mathrm{~A})$ & 109.5 \\
\hline $\mathrm{C}(24)-\mathrm{C}(23)-\mathrm{C}(22)$ & $119.5(6)$ & N(4)-C(34)-H(34B) & 109.5 \\
\hline $\mathrm{C}(24)-\mathrm{C}(23)-\mathrm{H}(23)$ & 120.2 & $\mathrm{C}(35)-\mathrm{C}(34)-\mathrm{H}(34 \mathrm{~B})$ & 109.5 \\
\hline $\mathrm{C}(22)-\mathrm{C}(23)-\mathrm{H}(23)$ & 120.2 & $\mathrm{H}(34 \mathrm{~A})-\mathrm{C}(34)-\mathrm{H}(34 \mathrm{~B})$ & 108.1 \\
\hline $\mathrm{C}(23)-\mathrm{C}(24)-\mathrm{C}(25)$ & $121.0(6)$ & $\mathrm{N}(5)-\mathrm{C}(35)-\mathrm{C}(34)$ & $112.0(4)$ \\
\hline $\mathrm{C}(23)-\mathrm{C}(24)-\mathrm{H}(24)$ & 119.5 & $\mathrm{~N}(5)-\mathrm{C}(35)-\mathrm{H}(35 \mathrm{~A})$ & 109.2 \\
\hline $\mathrm{C}(25)-\mathrm{C}(24)-\mathrm{H}(24)$ & 119.5 & $\mathrm{C}(34)-\mathrm{C}(35)-\mathrm{H}(35 \mathrm{~A})$ & 109.2 \\
\hline $\mathrm{C}(24)-\mathrm{C}(25)-\mathrm{C}(20)$ & $120.4(6)$ & $\mathrm{N}(5)-\mathrm{C}(35)-\mathrm{H}(35 \mathrm{~B})$ & 109.2 \\
\hline $\mathrm{C}(24)-\mathrm{C}(25)-\mathrm{H}(25)$ & 119.8 & $\mathrm{C}(34)-\mathrm{C}(35)-\mathrm{H}(35 \mathrm{~B})$ & 109.2 \\
\hline $\mathrm{C}(20)-\mathrm{C}(25)-\mathrm{H}(25)$ & 119.8 & $\mathrm{H}(35 \mathrm{~A})-\mathrm{C}(35)-\mathrm{H}(35 \mathrm{~B})$ & 107.9 \\
\hline
\end{tabular}




\begin{tabular}{|c|c|c|c|}
\hline $\mathrm{N}(5)-\mathrm{C}(36)-\mathrm{C}(37)$ & $112.4(5)$ & $\mathrm{N}(6)-\mathrm{C}(42)-\mathrm{H}(42 \mathrm{~A})$ & 109.5 \\
\hline $\mathrm{N}(5)-\mathrm{C}(36)-\mathrm{H}(36 \mathrm{~A})$ & 109.1 & $\mathrm{~N}(6)-\mathrm{C}(42)-\mathrm{H}(42 \mathrm{~B})$ & 109.5 \\
\hline $\mathrm{C}(37)-\mathrm{C}(36)-\mathrm{H}(36 \mathrm{~A})$ & 109.1 & $\mathrm{H}(42 \mathrm{~A})-\mathrm{C}(42)-\mathrm{H}(42 \mathrm{~B})$ & 109.5 \\
\hline $\mathrm{N}(5)-\mathrm{C}(36)-\mathrm{H}(36 \mathrm{~B})$ & 109.1 & $\mathrm{~N}(6)-\mathrm{C}(42)-\mathrm{H}(42 \mathrm{C})$ & 109.5 \\
\hline $\mathrm{C}(37)-\mathrm{C}(36)-\mathrm{H}(36 \mathrm{~B})$ & 109.1 & $\mathrm{H}(42 \mathrm{~A})-\mathrm{C}(42)-\mathrm{H}(42 \mathrm{C})$ & 109.5 \\
\hline $\mathrm{H}(36 \mathrm{~A})-\mathrm{C}(36)-\mathrm{H}(36 \mathrm{~B})$ & 107.9 & $\mathrm{H}(42 \mathrm{~B})-\mathrm{C}(42)-\mathrm{H}(42 \mathrm{C})$ & 109.5 \\
\hline $\mathrm{C}(36)-\mathrm{C}(37)-\mathrm{N}(6)$ & $112.0(5)$ & $\mathrm{C}(44)-\mathrm{C}(43)-\mathrm{Rh}(2)$ & $169.9(4)$ \\
\hline $\mathrm{C}(36)-\mathrm{C}(37)-\mathrm{H}(37 \mathrm{~A})$ & 109.2 & $\mathrm{C}(44)-\mathrm{C}(43)-\operatorname{Ag}(2) \# 2$ & $74.2(3)$ \\
\hline $\mathrm{N}(6)-\mathrm{C}(37)-\mathrm{H}(37 \mathrm{~A})$ & 109.2 & $\operatorname{Rh}(2)-C(43)-\operatorname{Ag}(2) \# 2$ & $113.5(2)$ \\
\hline $\mathrm{C}(36)-\mathrm{C}(37)-\mathrm{H}(37 \mathrm{~B})$ & 109.2 & $\mathrm{C}(43)-\mathrm{C}(44)-\mathrm{C}(45)$ & $167.2(5)$ \\
\hline $\mathrm{N}(6)-\mathrm{C}(37)-\mathrm{H}(37 \mathrm{~B})$ & 109.2 & $\mathrm{C}(43)-\mathrm{C}(44)-\operatorname{Ag}(2) \# 2$ & $76.2(3)$ \\
\hline $\mathrm{H}(37 \mathrm{~A})-\mathrm{C}(37)-\mathrm{H}(37 \mathrm{~B})$ & 107.9 & $C(45)-C(44)-\operatorname{Ag}(2) \# 2$ & $116.6(3)$ \\
\hline $\mathrm{N}(6)-\mathrm{C}(38)-\mathrm{C}(39)$ & $113.0(5)$ & $\mathrm{C}(50)-\mathrm{C}(45)-\mathrm{C}(46)$ & $117.7(5)$ \\
\hline $\mathrm{N}(6)-\mathrm{C}(38)-\mathrm{H}(38 \mathrm{~A})$ & 109.0 & $C(50)-C(45)-C(44)$ & $120.8(5)$ \\
\hline $\mathrm{C}(39)-\mathrm{C}(38)-\mathrm{H}(38 \mathrm{~A})$ & 109.0 & $\mathrm{C}(46)-\mathrm{C}(45)-\mathrm{C}(44)$ & $121.5(5)$ \\
\hline $\mathrm{N}(6)-\mathrm{C}(38)-\mathrm{H}(38 \mathrm{~B})$ & 109.0 & $\mathrm{C}(47)-\mathrm{C}(46)-\mathrm{C}(45)$ & $120.2(6)$ \\
\hline $\mathrm{C}(39)-\mathrm{C}(38)-\mathrm{H}(38 \mathrm{~B})$ & 109.0 & $\mathrm{C}(47)-\mathrm{C}(46)-\mathrm{H}(46)$ & 119.9 \\
\hline $\mathrm{H}(38 \mathrm{~A})-\mathrm{C}(38)-\mathrm{H}(38 \mathrm{~B})$ & 107.8 & $\mathrm{C}(45)-\mathrm{C}(46)-\mathrm{H}(46)$ & 119.9 \\
\hline $\mathrm{C}(38)-\mathrm{C}(39)-\mathrm{N}(4)$ & $110.8(4)$ & $\mathrm{C}(48)-\mathrm{C}(47)-\mathrm{C}(46)$ & $121.8(7)$ \\
\hline $\mathrm{C}(38)-\mathrm{C}(39)-\mathrm{H}(39 \mathrm{~A})$ & 109.5 & $\mathrm{C}(48)-\mathrm{C}(47)-\mathrm{H}(47)$ & 119.1 \\
\hline $\mathrm{N}(4)-\mathrm{C}(39)-\mathrm{H}(39 \mathrm{~A})$ & 109.5 & $\mathrm{C}(46)-\mathrm{C}(47)-\mathrm{H}(47)$ & 119.1 \\
\hline $\mathrm{C}(38)-\mathrm{C}(39)-\mathrm{H}(39 \mathrm{~B})$ & 109.5 & $\mathrm{C}(49)-\mathrm{C}(48)-\mathrm{C}(47)$ & $118.0(6)$ \\
\hline $\mathrm{N}(4)-\mathrm{C}(39)-\mathrm{H}(39 \mathrm{~B})$ & 109.5 & $\mathrm{C}(49)-\mathrm{C}(48)-\mathrm{H}(48)$ & 121.0 \\
\hline $\mathrm{H}(39 \mathrm{~A})-\mathrm{C}(39)-\mathrm{H}(39 \mathrm{~B})$ & 108.1 & $\mathrm{C}(47)-\mathrm{C}(48)-\mathrm{H}(48)$ & 121.0 \\
\hline $\mathrm{N}(4)-\mathrm{C}(40)-\mathrm{H}(40 \mathrm{~A})$ & 109.5 & $\mathrm{C}(48)-\mathrm{C}(49)-\mathrm{C}(50)$ & $122.2(6)$ \\
\hline $\mathrm{N}(4)-\mathrm{C}(40)-\mathrm{H}(40 \mathrm{~B})$ & 109.5 & $\mathrm{C}(48)-\mathrm{C}(49)-\mathrm{H}(49)$ & 118.9 \\
\hline $\mathrm{H}(40 \mathrm{~A})-\mathrm{C}(40)-\mathrm{H}(40 \mathrm{~B})$ & 109.5 & $\mathrm{C}(50)-\mathrm{C}(49)-\mathrm{H}(49)$ & 118.9 \\
\hline $\mathrm{N}(4)-\mathrm{C}(40)-\mathrm{H}(40 \mathrm{C})$ & 109.5 & $\mathrm{C}(45)-\mathrm{C}(50)-\mathrm{C}(49)$ & $120.0(6)$ \\
\hline $\mathrm{H}(40 \mathrm{~A})-\mathrm{C}(40)-\mathrm{H}(40 \mathrm{C})$ & 109.5 & $\mathrm{C}(45)-\mathrm{C}(50)-\mathrm{H}(50)$ & 120.0 \\
\hline $\mathrm{H}(40 \mathrm{~B})-\mathrm{C}(40)-\mathrm{H}(40 \mathrm{C})$ & 109.5 & $\mathrm{C}(49)-\mathrm{C}(50)-\mathrm{H}(50)$ & 120.0 \\
\hline $\mathrm{N}(5)-\mathrm{C}(41)-\mathrm{H}(41 \mathrm{~A})$ & 109.5 & $\mathrm{C}(52)-\mathrm{C}(51)-\mathrm{Rh}(2)$ & $174.8(4)$ \\
\hline $\mathrm{N}(5)-\mathrm{C}(41)-\mathrm{H}(41 \mathrm{~B})$ & 109.5 & $\mathrm{C}(52)-\mathrm{C}(51)-\mathrm{Ag}(2)$ & $81.3(3)$ \\
\hline $\mathrm{H}(41 \mathrm{~A})-\mathrm{C}(41)-\mathrm{H}(41 \mathrm{~B})$ & 109.5 & $\mathrm{Rh}(2)-\mathrm{C}(51)-\operatorname{Ag}(2)$ & $100.28(18)$ \\
\hline $\mathrm{N}(5)-\mathrm{C}(41)-\mathrm{H}(41 \mathrm{C})$ & 109.5 & $\mathrm{C}(51)-\mathrm{C}(52)-\mathrm{C}(53)$ & $171.7(5)$ \\
\hline $\mathrm{H}(41 \mathrm{~A})-\mathrm{C}(41)-\mathrm{H}(41 \mathrm{C})$ & 109.5 & $\mathrm{C}(51)-\mathrm{C}(52)-\mathrm{Ag}(2)$ & $70.9(3)$ \\
\hline $\mathrm{H}(41 \mathrm{~B})-\mathrm{C}(41)-\mathrm{H}(41 \mathrm{C})$ & 109.5 & $C(53)-C(52)-A g(2)$ & $116.0(3)$ \\
\hline
\end{tabular}




\begin{tabular}{|c|c|c|c|}
\hline $\mathrm{C}(54)-\mathrm{C}(53)-\mathrm{C}(58)$ & $118.7(5)$ & $\mathrm{C}(64)-\mathrm{C}(63)-\mathrm{C}(62)$ & $120.2(5)$ \\
\hline $\mathrm{C}(54)-\mathrm{C}(53)-\mathrm{C}(52)$ & $120.1(5)$ & $\mathrm{C}(64)-\mathrm{C}(63)-\mathrm{H}(63)$ & 119.9 \\
\hline $\mathrm{C}(58)-\mathrm{C}(53)-\mathrm{C}(52)$ & $121.2(4)$ & $\mathrm{C}(62)-\mathrm{C}(63)-\mathrm{H}(63)$ & 119.9 \\
\hline $\mathrm{C}(55)-\mathrm{C}(54)-\mathrm{C}(53)$ & $119.3(6)$ & $\mathrm{C}(65)-\mathrm{C}(64)-\mathrm{C}(63)$ & $120.3(5)$ \\
\hline $\mathrm{C}(55)-\mathrm{C}(54)-\mathrm{H}(54)$ & 120.4 & $\mathrm{C}(65)-\mathrm{C}(64)-\mathrm{H}(64)$ & 119.9 \\
\hline $\mathrm{C}(53)-\mathrm{C}(54)-\mathrm{H}(54)$ & 120.4 & $\mathrm{C}(63)-\mathrm{C}(64)-\mathrm{H}(64)$ & 119.9 \\
\hline$C(56)-C(55)-C(54)$ & $121.1(6)$ & $\mathrm{C}(64)-\mathrm{C}(65)-\mathrm{C}(66)$ & $120.0(5)$ \\
\hline $\mathrm{C}(56)-\mathrm{C}(55)-\mathrm{H}(55)$ & 119.4 & $\mathrm{C}(64)-\mathrm{C}(65)-\mathrm{H}(65)$ & 120.0 \\
\hline $\mathrm{C}(54)-\mathrm{C}(55)-\mathrm{H}(55)$ & 119.4 & $\mathrm{C}(66)-\mathrm{C}(65)-\mathrm{H}(65)$ & 120.0 \\
\hline$C(57)-C(56)-C(55)$ & $119.6(6)$ & $\mathrm{C}(65)-\mathrm{C}(66)-\mathrm{C}(61)$ & $120.4(5)$ \\
\hline $\mathrm{C}(57)-\mathrm{C}(56)-\mathrm{H}(56)$ & 120.2 & $\mathrm{C}(65)-\mathrm{C}(66)-\mathrm{H}(66)$ & 119.8 \\
\hline $\mathrm{C}(55)-\mathrm{C}(56)-\mathrm{H}(56)$ & 120.2 & $\mathrm{C}(61)-\mathrm{C}(66)-\mathrm{H}(66)$ & 119.8 \\
\hline $\mathrm{C}(56)-\mathrm{C}(57)-\mathrm{C}(58)$ & $120.6(6)$ & $\mathrm{N}(7)-\mathrm{C}(67)-\mathrm{C}(68)$ & $177.7(13)$ \\
\hline $\mathrm{C}(56)-\mathrm{C}(57)-\mathrm{H}(57)$ & 119.7 & $\mathrm{C}(67)-\mathrm{C}(68)-\mathrm{H}(68 \mathrm{~A})$ & 109.5 \\
\hline $\mathrm{C}(58)-\mathrm{C}(57)-\mathrm{H}(57)$ & 119.7 & $\mathrm{C}(67)-\mathrm{C}(68)-\mathrm{H}(68 \mathrm{~B})$ & 109.5 \\
\hline $\mathrm{C}(57)-\mathrm{C}(58)-\mathrm{C}(53)$ & $120.8(5)$ & $\mathrm{H}(68 \mathrm{~A})-\mathrm{C}(68)-\mathrm{H}(68 \mathrm{~B})$ & 109.5 \\
\hline $\mathrm{C}(57)-\mathrm{C}(58)-\mathrm{H}(58)$ & 119.6 & $\mathrm{C}(67)-\mathrm{C}(68)-\mathrm{H}(68 \mathrm{C})$ & 109.5 \\
\hline $\mathrm{C}(53)-\mathrm{C}(58)-\mathrm{H}(58)$ & 119.6 & $\mathrm{H}(68 \mathrm{~A})-\mathrm{C}(68)-\mathrm{H}(68 \mathrm{C})$ & 109.5 \\
\hline $\mathrm{C}(60)-\mathrm{C}(59)-\mathrm{Rh}(2)$ & $175.7(4)$ & $\mathrm{H}(68 \mathrm{~B})-\mathrm{C}(68)-\mathrm{H}(68 \mathrm{C})$ & 109.5 \\
\hline$C(60)-C(59)-\operatorname{Ag}(2)$ & $79.7(3)$ & $\mathrm{F}(1)-\mathrm{B}-\mathrm{F}(4)$ & $115.8(8)$ \\
\hline $\operatorname{Rh}(2)-C(59)-\operatorname{Ag}(2)$ & $100.67(18)$ & $\mathrm{F}(1)-\mathrm{B}-\mathrm{F}(3)$ & 108.1(7) \\
\hline $\mathrm{C}(59)-\mathrm{C}(60)-\mathrm{C}(61)$ & $166.0(5)$ & $\mathrm{F}(4)-\mathrm{B}-\mathrm{F}(3)$ & 107.1(6) \\
\hline$C(59)-C(60)-\operatorname{Ag}(2)$ & $71.6(3)$ & $\mathrm{F}(1)-\mathrm{B}-\mathrm{F}(2)$ & $109.5(5)$ \\
\hline$C(61)-C(60)-\operatorname{Ag}(2)$ & $122.4(3)$ & $\mathrm{F}(4)-\mathrm{B}-\mathrm{F}(2)$ & 109.0(7) \\
\hline$C(66)-C(61)-C(62)$ & $119.2(4)$ & $\mathrm{F}(3)-\mathrm{B}-\mathrm{F}(2)$ & $107.0(7)$ \\
\hline$C(66)-C(61)-C(60)$ & $119.9(5)$ & & \\
\hline$C(62)-C(61)-C(60)$ & $120.9(4)$ & \multirow{2}{*}{\multicolumn{2}{|c|}{ Symmetry transformations used to generate }} \\
\hline$C(63)-C(62)-C(61)$ & $119.9(5)$ & & \\
\hline $\mathrm{C}(63)-\mathrm{C}(62)-\mathrm{H}(62)$ & 120.0 & \multirow{2}{*}{\multicolumn{2}{|c|}{$\# 1-x+2,-y+2,-z+2 \quad \# 2-x+1,-y+1,-z+1$}} \\
\hline $\mathrm{C}(61)-\mathrm{C}(62)-\mathrm{H}(62)$ & 120.0 & & \\
\hline
\end{tabular}


Table 4. Anisotropic displacement parameters $\left(\AA^{2} \times 10^{3}\right)$ for 3. The anisotropic displacement factor exponent takes the form: $-2 \pi^{2}\left[h^{2} a^{* 2} U^{11}+\ldots+2 h k a^{*} b^{*} U^{12}\right]$

\begin{tabular}{|c|c|c|c|c|c|c|}
\hline & $\mathrm{U}^{11}$ & $\mathrm{U}^{22}$ & $\mathrm{U}^{33}$ & $\mathrm{U}^{23}$ & $\mathrm{U}^{13}$ & $\mathrm{U}^{12}$ \\
\hline $\operatorname{Ag}(1)$ & $45(1)$ & $34(1)$ & $35(1)$ & $-17(1)$ & $-10(1)$ & $-14(1)$ \\
\hline $\operatorname{Ag}(2)$ & $27(1)$ & $28(1)$ & $32(1)$ & $-6(1)$ & $-14(1)$ & $-5(1)$ \\
\hline $\mathrm{Rh}(1)$ & $45(1)$ & $28(1)$ & $28(1)$ & $-12(1)$ & $-12(1)$ & $-15(1)$ \\
\hline $\operatorname{Rh}(2)$ & $22(1)$ & $27(1)$ & $25(1)$ & $-8(1)$ & $-9(1)$ & $-6(1)$ \\
\hline $\mathrm{F}(1)$ & $228(7)$ & $88(4)$ & $66(3)$ & $21(3)$ & $-64(4)$ & $-101(4)$ \\
\hline $\mathrm{F}(2)$ & $120(4)$ & $94(3)$ & $47(2)$ & $-9(2)$ & $-21(2)$ & $-77(3)$ \\
\hline $\mathrm{F}(3)$ & $71(3)$ & $110(4)$ & $68(3)$ & 6(3) & $-31(2)$ & $-27(3)$ \\
\hline $\mathrm{F}(4)$ & $71(3)$ & $114(4)$ & $75(3)$ & $-42(3)$ & $-44(2)$ & $2(3)$ \\
\hline $\mathrm{N}(1)$ & $45(3)$ & $32(2)$ & $33(2)$ & $-14(2)$ & $-17(2)$ & $-10(2)$ \\
\hline $\mathrm{N}(2)$ & $71(3)$ & $29(2)$ & $35(2)$ & $-11(2)$ & $-24(2)$ & $-13(2)$ \\
\hline $\mathrm{N}(3)$ & $69(3)$ & $45(3)$ & $37(2)$ & $-9(2)$ & $-16(2)$ & $-32(3)$ \\
\hline $\mathrm{N}(4)$ & $37(2)$ & $37(2)$ & $28(2)$ & $-9(2)$ & $-11(2)$ & $-6(2)$ \\
\hline $\mathrm{N}(5)$ & $26(2)$ & $51(3)$ & $40(2)$ & $-10(2)$ & $-16(2)$ & $-12(2)$ \\
\hline $\mathrm{N}(6)$ & $36(2)$ & $36(2)$ & $41(2)$ & $-15(2)$ & $-16(2)$ & $-9(2)$ \\
\hline $\mathrm{N}(7)$ & $88(7)$ & $127(9)$ & $140(9)$ & $-51(7)$ & $-31(6)$ & $-29(6)$ \\
\hline $\mathrm{C}(1)$ & $59(4)$ & $37(3)$ & $36(3)$ & $-17(2)$ & $-19(3)$ & $-12(3)$ \\
\hline$C(2)$ & $60(4)$ & $31(3)$ & $39(3)$ & $-17(2)$ & $-19(3)$ & $-6(3)$ \\
\hline$C(3)$ & $88(5)$ & $32(3)$ & 49(3) & $-3(2)$ & $-33(3)$ & $-24(3)$ \\
\hline$C(4)$ & $91(5)$ & $54(4)$ & $40(3)$ & $-4(3)$ & $-23(3)$ & $-43(4)$ \\
\hline$C(5)$ & $56(4)$ & $61(4)$ & $44(3)$ & $-17(3)$ & $-17(3)$ & $-28(3)$ \\
\hline$C(6)$ & $54(4)$ & $52(3)$ & $46(3)$ & $-19(3)$ & $-26(3)$ & $-15(3)$ \\
\hline$C(7)$ & $60(4)$ & $37(3)$ & $40(3)$ & $-12(2)$ & $-24(3)$ & $-11(3)$ \\
\hline $\mathrm{C}(8)$ & $84(5)$ & $30(3)$ & $49(3)$ & $-15(3)$ & $-36(3)$ & $3(3)$ \\
\hline $\mathrm{C}(9)$ & $59(4)$ & $72(4)$ & $42(3)$ & $-18(3)$ & $-10(3)$ & $-35(4)$ \\
\hline$C(10)$ & $40(3)$ & $30(2)$ & $31(2)$ & $-14(2)$ & $-11(2)$ & $-11(2)$ \\
\hline $\mathrm{C}(11)$ & $54(3)$ & $32(3)$ & $37(3)$ & $-16(2)$ & $-17(3)$ & $-10(2)$ \\
\hline $\mathrm{C}(12)$ & $47(3)$ & $28(3)$ & $43(3)$ & $-14(2)$ & $-11(3)$ & $-5(2)$ \\
\hline$C(13)$ & $58(4)$ & $52(4)$ & $50(3)$ & $-14(3)$ & $-12(3)$ & $-15(3)$ \\
\hline$C(14)$ & $62(4)$ & $54(4)$ & $50(4)$ & $-16(3)$ & $-1(3)$ & $-12(3)$ \\
\hline$C(15)$ & $47(4)$ & $39(3)$ & $72(5)$ & $-16(3)$ & $-5(3)$ & $-11(3)$ \\
\hline$C(16)$ & $46(4)$ & $68(5)$ & $76(5)$ & $-27(4)$ & $-15(3)$ & $-15(3)$ \\
\hline
\end{tabular}




\begin{tabular}{|c|c|c|c|c|c|c|}
\hline $\mathrm{C}(17)$ & $51(4)$ & $59(4)$ & $58(4)$ & $-25(3)$ & $-15(3)$ & $-13(3)$ \\
\hline $\mathrm{C}(18)$ & $46(3)$ & $26(2)$ & $39(3)$ & $-15(2)$ & $-10(2)$ & $-14(2)$ \\
\hline C(19) & $51(3)$ & $31(3)$ & $33(3)$ & $-12(2)$ & $-10(2)$ & $-18(2)$ \\
\hline $\mathrm{C}(20)$ & $39(3)$ & $36(3)$ & $33(3)$ & $-14(2)$ & $-10(2)$ & $-5(2)$ \\
\hline $\mathrm{C}(21)$ & $124(7)$ & $54(4)$ & $44(3)$ & $-7(3)$ & $-41(4)$ & $-38(4)$ \\
\hline $\mathrm{C}(22)$ & $122(7)$ & $62(5)$ & $45(4)$ & $-15(3)$ & $-41(4)$ & $-17(5)$ \\
\hline $\mathrm{C}(23)$ & $57(4)$ & $62(4)$ & $30(3)$ & $-8(3)$ & $-10(3)$ & $12(3)$ \\
\hline $\mathrm{C}(24)$ & $46(3)$ & $44(3)$ & $50(3)$ & $3(3)$ & $-15(3)$ & $0(3)$ \\
\hline$C(25)$ & $40(3)$ & $36(3)$ & $43(3)$ & $-4(2)$ & $-15(2)$ & $-6(2)$ \\
\hline$C(26)$ & $39(3)$ & $35(3)$ & $31(2)$ & $-16(2)$ & $-11(2)$ & $-10(2)$ \\
\hline $\mathrm{C}(27)$ & $49(3)$ & $44(3)$ & $38(3)$ & $-16(2)$ & $-14(3)$ & $-15(3)$ \\
\hline $\mathrm{C}(28)$ & $45(3)$ & $45(3)$ & $38(3)$ & $-15(2)$ & $-16(2)$ & $-6(3)$ \\
\hline $\mathrm{C}(29)$ & $57(4)$ & $48(4)$ & $55(4)$ & $-14(3)$ & $-26(3)$ & $-8(3)$ \\
\hline $\mathrm{C}(30)$ & $45(3)$ & $65(4)$ & $54(4)$ & $-11(3)$ & $-20(3)$ & $-8(3)$ \\
\hline $\mathrm{C}(31)$ & $61(4)$ & $46(3)$ & 49(3) & $-18(3)$ & $-31(3)$ & $6(3)$ \\
\hline$C(32)$ & $62(4)$ & $38(3)$ & $43(3)$ & $-15(2)$ & $-27(3)$ & $-3(3)$ \\
\hline $\mathrm{C}(33)$ & $47(3)$ & $37(3)$ & $37(3)$ & $-11(2)$ & $-22(2)$ & $-4(2)$ \\
\hline$C(34)$ & $40(3)$ & $52(4)$ & $36(3)$ & $-7(3)$ & $-15(2)$ & $0(3)$ \\
\hline $\mathrm{C}(35)$ & $39(3)$ & $60(4)$ & 49(3) & $-9(3)$ & $-26(3)$ & $-6(3)$ \\
\hline$C(36)$ & $40(3)$ & $56(4)$ & $58(4)$ & $-12(3)$ & $-18(3)$ & $-24(3)$ \\
\hline $\mathrm{C}(37)$ & $47(3)$ & 51(4) & $65(4)$ & $-23(3)$ & $-24(3)$ & $-18(3)$ \\
\hline $\mathrm{C}(38)$ & $46(3)$ & $53(3)$ & $40(3)$ & $-24(3)$ & $-10(3)$ & $-9(3)$ \\
\hline C(39) & $44(3)$ & $52(3)$ & $31(3)$ & $-15(2)$ & $-11(2)$ & $-6(3)$ \\
\hline $\mathrm{C}(40)$ & $54(4)$ & $44(3)$ & $33(3)$ & $-3(2)$ & $-14(3)$ & $-17(3)$ \\
\hline $\mathrm{C}(41)$ & $26(3)$ & $76(4)$ & $45(3)$ & $-15(3)$ & $-11(2)$ & $-15(3)$ \\
\hline $\mathrm{C}(42)$ & $56(4)$ & $36(3)$ & 59(4) & $-24(3)$ & $-28(3)$ & $1(3)$ \\
\hline $\mathrm{C}(43)$ & $27(2)$ & $30(2)$ & $20(2)$ & $-2(2)$ & $-11(2)$ & $-6(2)$ \\
\hline $\mathrm{C}(44)$ & $28(2)$ & $34(3)$ & $25(2)$ & $-6(2)$ & $-9(2)$ & $-5(2)$ \\
\hline $\mathrm{C}(45)$ & $34(3)$ & $31(3)$ & $34(2)$ & $-11(2)$ & $-13(2)$ & $0(2)$ \\
\hline$C(46)$ & $45(4)$ & $41(3)$ & $89(5)$ & $-20(3)$ & $-23(4)$ & $-5(3)$ \\
\hline $\mathrm{C}(47)$ & $71(5)$ & $42(4)$ & $95(6)$ & $-19(4)$ & $-42(4)$ & $-6(3)$ \\
\hline $\mathrm{C}(48)$ & $69(5)$ & $35(3)$ & $73(5)$ & $-20(3)$ & $-33(4)$ & $7(3)$ \\
\hline C(49) & $42(3)$ & $49(4)$ & $63(4)$ & $-19(3)$ & $-20(3)$ & $14(3)$ \\
\hline $\mathrm{C}(50)$ & $36(3)$ & $40(3)$ & $47(3)$ & $-12(2)$ & $-12(2)$ & $-2(2)$ \\
\hline $\mathrm{C}(51)$ & $22(2)$ & $27(2)$ & $35(2)$ & $-10(2)$ & $-9(2)$ & $-8(2)$ \\
\hline $\mathrm{C}(52)$ & $23(2)$ & $33(3)$ & $39(3)$ & $-14(2)$ & $-10(2)$ & $-6(2)$ \\
\hline
\end{tabular}




\begin{tabular}{lllllll}
$\mathrm{C}(53)$ & $21(2)$ & $28(2)$ & $37(3)$ & $-7(2)$ & $-5(2)$ & $-7(2)$ \\
$\mathrm{C}(54)$ & $34(3)$ & $35(3)$ & $47(3)$ & $-16(2)$ & $-6(2)$ & $-9(2)$ \\
$\mathrm{C}(55)$ & $47(3)$ & $31(3)$ & $67(4)$ & $-8(3)$ & $-1(3)$ & $-14(3)$ \\
$\mathrm{C}(56)$ & $55(4)$ & $36(3)$ & $51(4)$ & $2(3)$ & $-3(3)$ & $-11(3)$ \\
$\mathrm{C}(57)$ & $46(3)$ & $43(3)$ & $42(3)$ & $-3(2)$ & $-9(3)$ & $-13(3)$ \\
$\mathrm{C}(58)$ & $35(3)$ & $33(3)$ & $39(3)$ & $-4(2)$ & $-11(2)$ & $-11(2)$ \\
$\mathrm{C}(59)$ & $29(2)$ & $24(2)$ & $26(2)$ & $-10(2)$ & $-10(2)$ & $-7(2)$ \\
$\mathrm{C}(60)$ & $31(2)$ & $26(2)$ & $22(2)$ & $-8(2)$ & $-7(2)$ & $-10(2)$ \\
$\mathrm{C}(61)$ & $23(2)$ & $28(2)$ & $32(2)$ & $-13(2)$ & $-4(2)$ & $-8(2)$ \\
$\mathrm{C}(62)$ & $26(2)$ & $44(3)$ & $34(2)$ & $-18(2)$ & $-6(2)$ & $-10(2)$ \\
$\mathrm{C}(63)$ & $26(2)$ & $52(3)$ & $44(3)$ & $-19(2)$ & $-15(2)$ & $-11(2)$ \\
$\mathrm{C}(64)$ & $24(2)$ & $47(3)$ & $48(3)$ & $-23(3)$ & $1(2)$ & $-14(2)$ \\
$\mathrm{C}(65)$ & $38(3)$ & $36(3)$ & $40(3)$ & $-12(2)$ & $0(2)$ & $-16(2)$ \\
$\mathrm{C}(66)$ & $35(3)$ & $29(2)$ & $33(2)$ & $-7(2)$ & $-8(2)$ & $-8(2)$ \\
$\mathrm{C}(67)$ & $87(8)$ & $104(9)$ & $90(7)$ & $-19(6)$ & $-39(6)$ & $6(6)$ \\
$\mathrm{C}(68)$ & $126(10)$ & $134(11)$ & $116(10)$ & $60(8)$ & $-36(8)$ & $-4(8)$ \\
$\mathrm{B}$ & $92(6)$ & $65(5)$ & $44(4)$ & $-4(3)$ & $-32(4)$ & $-45(5)$ \\
& & & & & & \\
\hline
\end{tabular}


Table 5. Hydrogen coordinates ( $\left.\mathrm{x} 10^{4}\right)$ and isotropic displacement parameters $\left(\AA^{2} \mathrm{x} 10^{3}\right)$ for 3 .

\begin{tabular}{|c|c|c|c|c|}
\hline & $\mathrm{x}$ & $\mathrm{y}$ & $\mathrm{z}$ & $\mathrm{U}(\mathrm{eq})$ \\
\hline $\mathrm{H}(1 \mathrm{~A})$ & 9503 & 7534 & 7539 & 49 \\
\hline $\mathrm{H}(1 \mathrm{~B})$ & 10379 & 8019 & 6833 & 49 \\
\hline $\mathrm{H}(2 \mathrm{~A})$ & 11160 & 6784 & 7707 & 50 \\
\hline $\mathrm{H}(2 \mathrm{~B})$ & 11473 & 7663 & 7683 & 50 \\
\hline $\mathrm{H}(3 \mathrm{~A})$ & 9375 & 6680 & 8722 & 61 \\
\hline $\mathrm{H}(3 \mathrm{~B})$ & 9882 & 6212 & 9483 & 61 \\
\hline $\mathrm{H}(4 \mathrm{~A})$ & 7949 & 6915 & 10004 & 66 \\
\hline $\mathrm{H}(4 \mathrm{~B})$ & 8665 & 7216 & 10411 & 66 \\
\hline $\mathrm{H}(5 \mathrm{~A})$ & 8007 & 7637 & 8553 & 59 \\
\hline $\mathrm{H}(5 \mathrm{~B})$ & 6919 & 8369 & 8974 & 59 \\
\hline $\mathrm{H}(6 \mathrm{~A})$ & 7756 & 9470 & 8247 & 55 \\
\hline $\mathrm{H}(6 \mathrm{~B})$ & 7994 & 8839 & 7539 & 55 \\
\hline $\mathrm{H}(7 \mathrm{~A})$ & 9521 & 9489 & 6702 & 63 \\
\hline $\mathrm{H}(7 \mathrm{~B})$ & 10467 & 9412 & 7090 & 63 \\
\hline $\mathrm{H}(7 \mathrm{C})$ & 9257 & 10020 & 7495 & 63 \\
\hline $\mathrm{H}(8 \mathrm{~A})$ & 11727 & 6365 & 9010 & 79 \\
\hline $\mathrm{H}(8 \mathrm{~B})$ & 11018 & 6862 & 9858 & 79 \\
\hline $\mathrm{H}(8 \mathrm{C})$ & 11808 & 7321 & 9032 & 79 \\
\hline $\mathrm{H}(9 \mathrm{~A})$ & 6549 & 8314 & 10431 & 80 \\
\hline $\mathrm{H}(9 \mathrm{~B})$ & 6899 & 9210 & 10052 & 80 \\
\hline $\mathrm{H}(9 \mathrm{C})$ & 7385 & 8552 & 10760 & 80 \\
\hline $\mathrm{H}(13)$ & 12639 & 9111 & 6492 & 64 \\
\hline $\mathrm{H}(14)$ & 14439 & 9014 & 5549 & 72 \\
\hline $\mathrm{H}(15)$ & 15808 & 9029 & 6054 & 67 \\
\hline $\mathrm{H}(16)$ & 15416 & 9060 & 7539 & 74 \\
\hline $\mathrm{H}(17)$ & 13642 & 9126 & 8497 & 65 \\
\hline $\mathrm{H}(21)$ & 9918 & 9030 & 12194 & 79 \\
\hline $\mathrm{H}(22)$ & 9846 & 8479 & 13630 & 87 \\
\hline $\mathrm{H}(23)$ & 9380 & 7160 & 14235 & 69 \\
\hline $\mathrm{H}(24)$ & 9045 & 6381 & 13398 & 61 \\
\hline
\end{tabular}




\begin{tabular}{|c|c|c|c|c|}
\hline $\mathrm{H}(25)$ & 9135 & 6904 & 11974 & 48 \\
\hline $\mathrm{H}(29)$ & 5849 & 10948 & 9890 & 61 \\
\hline $\mathrm{H}(30)$ & 4509 & 12266 & 10282 & 65 \\
\hline $\mathrm{H}(31)$ & 5044 & 13480 & 10299 & 61 \\
\hline $\mathrm{H}(32)$ & 6893 & 13411 & 9909 & 54 \\
\hline $\mathrm{H}(33)$ & 8236 & 12121 & 9517 & 46 \\
\hline $\mathrm{H}(34 \mathrm{~A})$ & 7370 & 3271 & 7139 & 54 \\
\hline $\mathrm{H}(34 \mathrm{~B})$ & 7201 & 3391 & 8116 & 54 \\
\hline $\mathrm{H}(35 \mathrm{~A})$ & 7729 & 4673 & 7683 & 56 \\
\hline $\mathrm{H}(35 \mathrm{~B})$ & 8629 & 4005 & 7019 & 56 \\
\hline $\mathrm{H}(36 \mathrm{~A})$ & 7475 & 6248 & 6041 & 56 \\
\hline $\mathrm{H}(36 \mathrm{~B})$ & 8158 & 5877 & 6703 & 56 \\
\hline $\mathrm{H}(37 \mathrm{~A})$ & 6366 & 6846 & 7286 & 59 \\
\hline $\mathrm{H}(37 \mathrm{~B})$ & 6611 & 5941 & 7878 & 59 \\
\hline $\mathrm{H}(38 \mathrm{~A})$ & 4164 & 5594 & 8110 & 54 \\
\hline $\mathrm{H}(38 \mathrm{~B})$ & 4681 & 6020 & 8587 & 54 \\
\hline $\mathrm{H}(39 \mathrm{~A})$ & 4983 & 4467 & 8923 & 51 \\
\hline $\mathrm{H}(39 \mathrm{~B})$ & 6086 & 4790 & 8584 & 51 \\
\hline $\mathrm{H}(40 \mathrm{~A})$ & 5556 & 3189 & 7394 & 64 \\
\hline $\mathrm{H}(40 \mathrm{~B})$ & 4519 & 3839 & 7995 & 64 \\
\hline $\mathrm{H}(40 \mathrm{C})$ & 5381 & 3137 & 8407 & 64 \\
\hline $\mathrm{H}(41 \mathrm{~A})$ & 8318 & 5109 & 5146 & 71 \\
\hline $\mathrm{H}(41 \mathrm{~B})$ & 8585 & 4115 & 5562 & 71 \\
\hline $\mathrm{H}(41 \mathrm{C})$ & 9190 & 4773 & 5657 & 71 \\
\hline $\mathrm{H}(42 \mathrm{~A})$ & 4102 & 6710 & 7008 & 72 \\
\hline $\mathrm{H}(42 \mathrm{~B})$ & 5143 & 7095 & 6465 & 72 \\
\hline $\mathrm{H}(42 \mathrm{C})$ & 4512 & 7245 & 7469 & 72 \\
\hline $\mathrm{H}(46)$ & 5871 & 2017 & 5594 & 69 \\
\hline $\mathrm{H}(47)$ & 6665 & 547 & 5464 & 79 \\
\hline $\mathrm{H}(48)$ & 8556 & -32 & 5115 & 71 \\
\hline $\mathrm{H}(49)$ & 9640 & 882 & 4907 & 66 \\
\hline $\mathrm{H}(50)$ & 8879 & 2356 & 5107 & 50 \\
\hline $\mathrm{H}(54)$ & 6581 & 7848 & 4750 & 47 \\
\hline $\mathrm{H}(55)$ & 6727 & 9011 & 3642 & 62 \\
\hline $\mathrm{H}(56)$ & 6507 & 8951 & 2335 & 64 \\
\hline $\mathrm{H}(57)$ & 6201 & 7708 & 2103 & 54 \\
\hline
\end{tabular}




\begin{tabular}{lrrrr}
$\mathrm{H}(58)$ & 6058 & 6539 & 3181 & 43 \\
$\mathrm{H}(62)$ & 1987 & 5827 & 5906 & 40 \\
$\mathrm{H}(63)$ & 109 & 5884 & 6466 & 45 \\
$\mathrm{H}(64)$ & -653 & 5281 & 7883 & 47 \\
$\mathrm{H}(65)$ & 452 & 4604 & 8747 & 47 \\
$\mathrm{H}(66)$ & 2319 & 4579 & 8215 & 39 \\
$\mathrm{H}(68 \mathrm{~A})$ & 2808 & 8810 & 2160 & 219 \\
$\mathrm{H}(68 \mathrm{~B})$ & 2924 & 9116 & 2965 & 219 \\
$\mathrm{H}(68 \mathrm{C})$ & 3763 & 8252 & 2551 & 219 \\
& & & & \\
\hline
\end{tabular}




\section{X-ray Structure of 4 Asymmetric Unit}

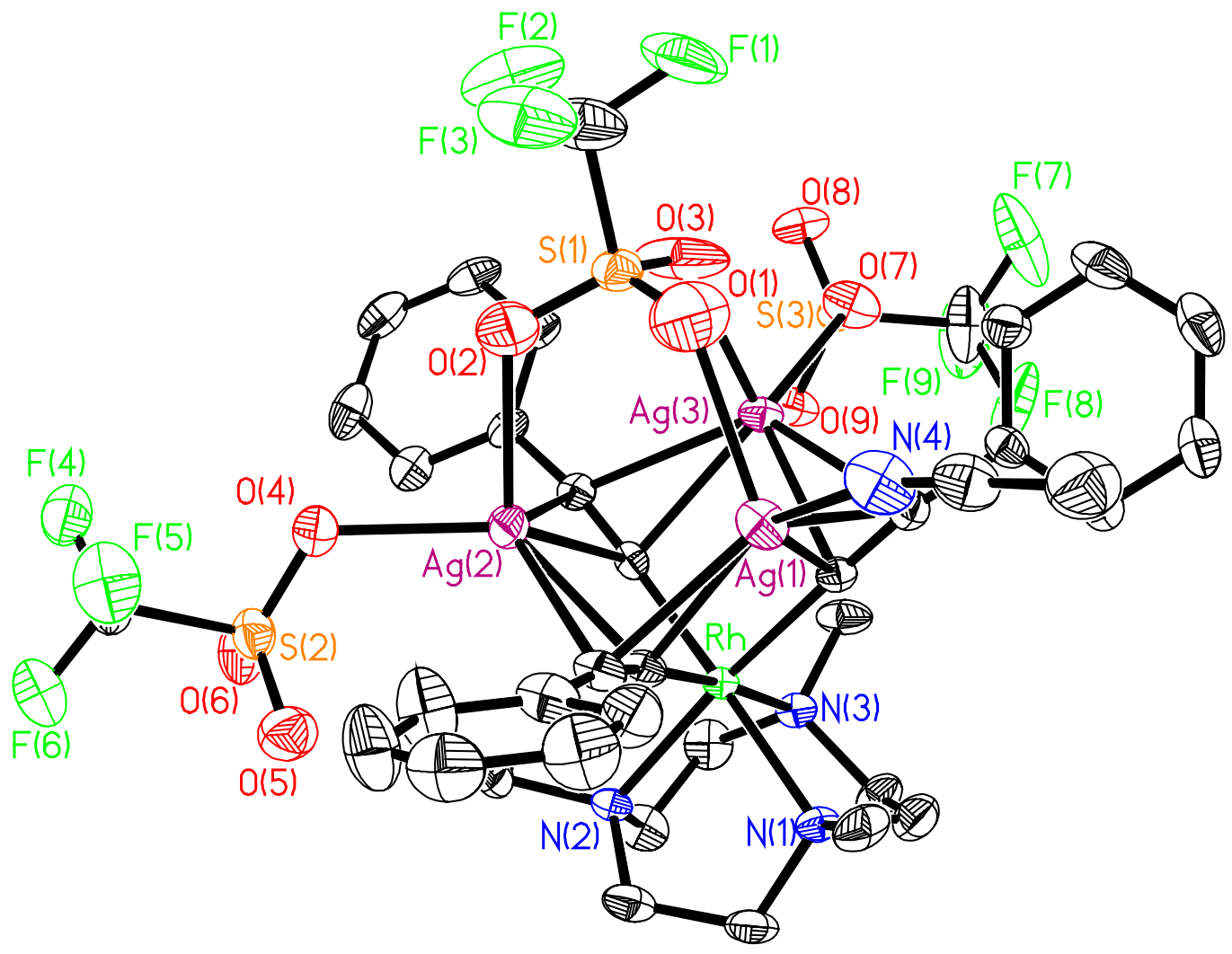




\section{Molecule}

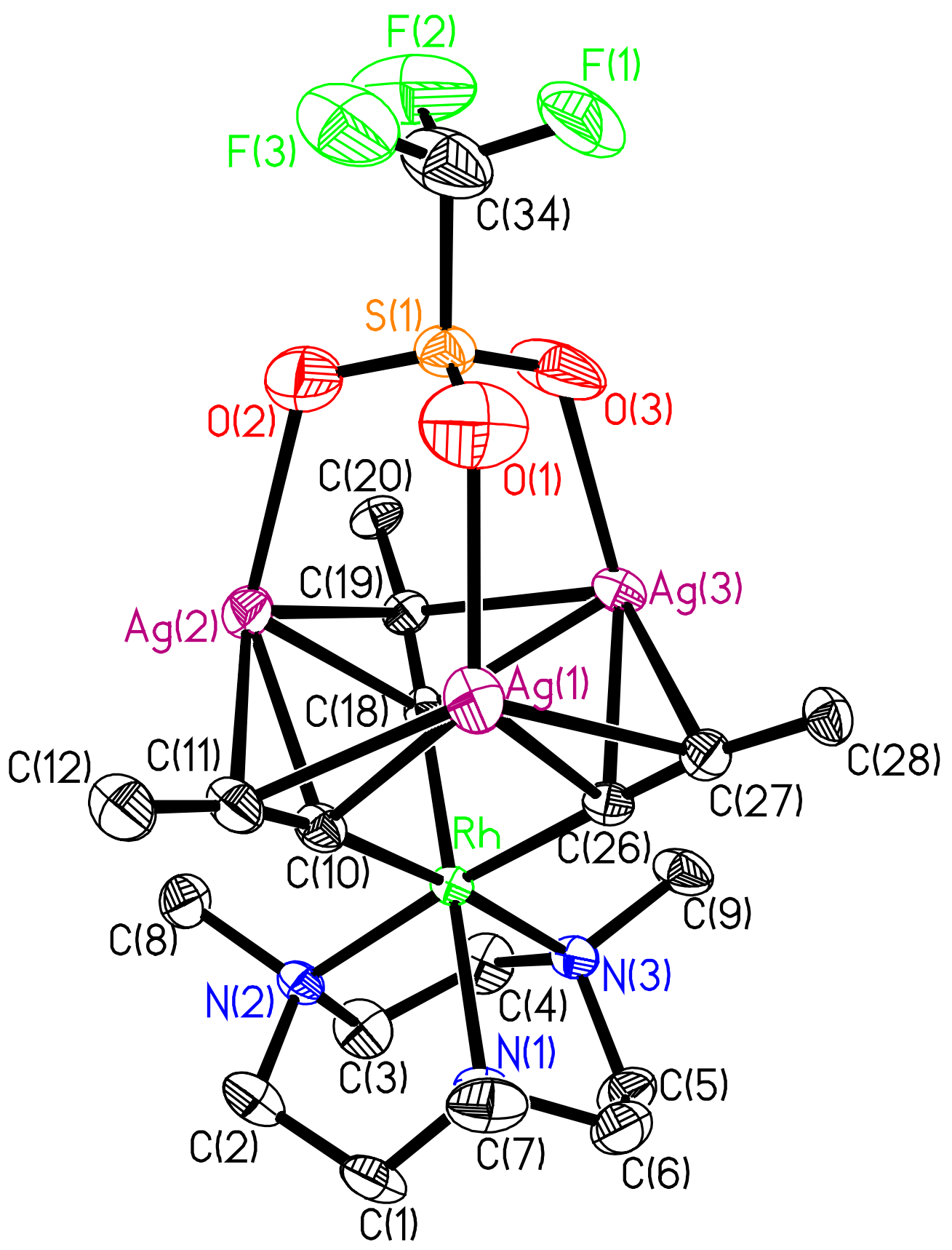




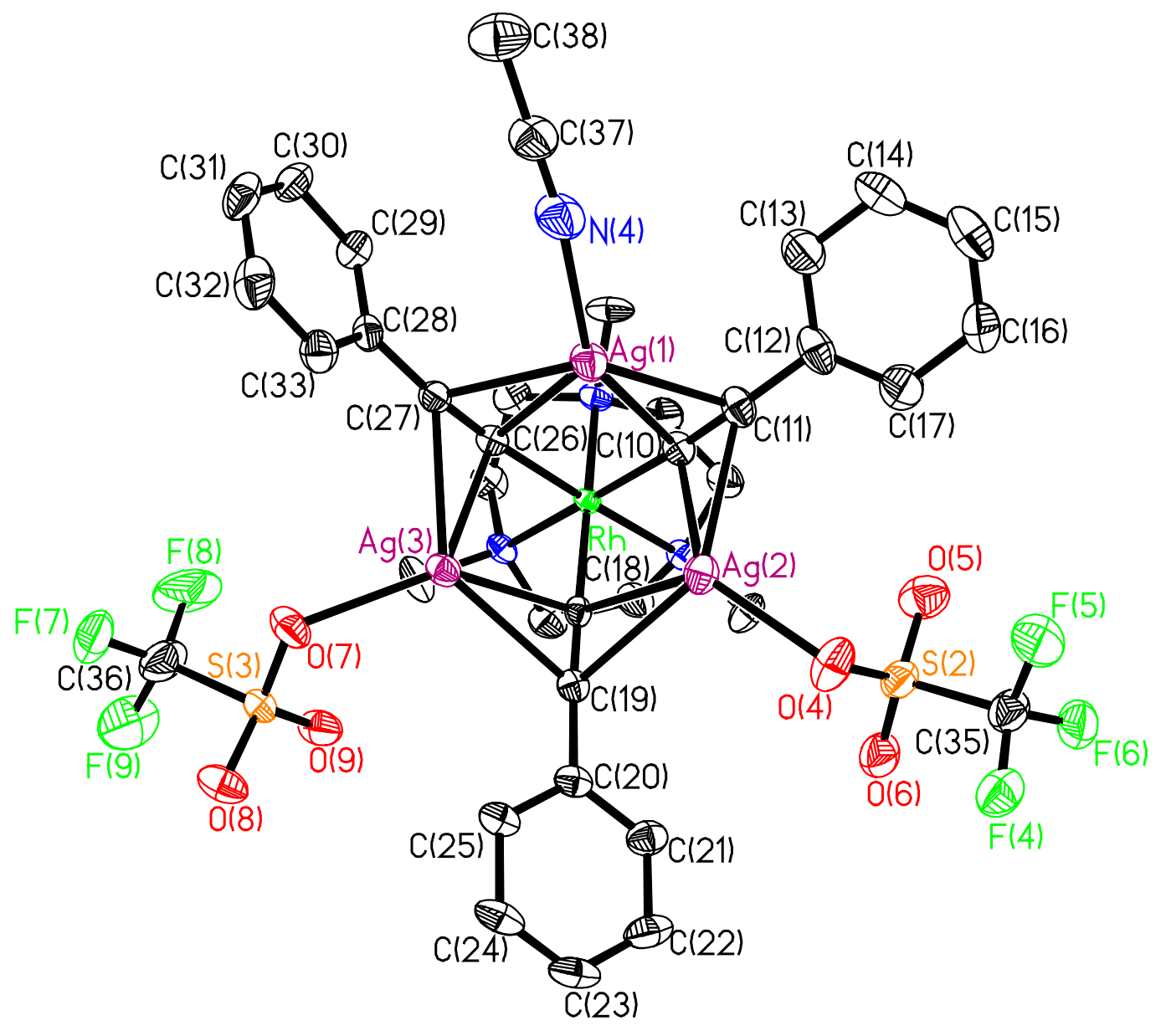


Table 1. Crystal data and structure refinement for 4 .

Identification code

4

Empirical formula

C38 H39 Ag3 F9 N4 O9 Rh S3

Formula weight

1389.43

Temperature

100(2) K

Wavelength

$0.71073 \AA$

Crystal system

Monoclinic

Space group

Unit cell dimensions

P 21/c

$\mathrm{a}=21.9776(19) \AA \quad \alpha=90^{\circ}$.

$\mathrm{b}=10.6829(9) \AA \quad \beta=113.5970(10)^{\circ}$.

$\mathrm{c}=21.8444(19) \AA \quad \gamma=90^{\circ}$.

Volume

4699.9(7) $\AA^{3}$

$\mathrm{Z}$

4

Density (calculated)

$1.964 \mathrm{Mg} / \mathrm{m}^{3}$

Absorption coefficient

$1.801 \mathrm{~mm}^{-1}$

$\mathrm{F}(000)$

2728

Crystal size

$0.25 \times 0.15 \times 0.07 \mathrm{~mm}^{3}$

Theta range for data collection

1.87 to $27.53^{\circ}$.

Index ranges

$-28 \leq \mathrm{h} \leq 28,-13 \leq \mathrm{k} \leq 13,-28 \leq 1 \leq 28$

Reflections collected

40140

Independent reflections

$10738[\mathrm{R}(\mathrm{int})=0.0380]$

Completeness to theta $=27.53^{\circ}$

$99.1 \%$

Absorption correction

Semi-empirical from equivalents

Max. and min. transmission

0.8843 and 0.7942

Refinement method

Full-matrix least-squares on $\mathrm{F}^{2}$

Data / restraints / parameters

10738 / 0 / 608

Goodness-of-fit on $\mathrm{F}^{2}$

1.099

Final $\mathrm{R}$ indices [I $>2 \operatorname{sigma}(\mathrm{I})]$

$\mathrm{R} 1=0.0510, \mathrm{wR} 2=0.1213$

$\mathrm{R}$ indices (all data)

$\mathrm{R} 1=0.0627, \mathrm{wR} 2=0.1270$

Largest diff. peak and hole

2.085 and -0.946 e. $\AA^{-3}$ 
Table 2. Atomic coordinates ( $\left.\mathrm{x} 10^{4}\right)$ and equivalent isotropic displacement parameters $\left(\AA^{2} \times 10^{3}\right)$ for 4. $U(e q)$ is defined as one third of the trace of the orthogonalized $U^{i j}$ tensor.

\begin{tabular}{|c|c|c|c|c|}
\hline & $\mathrm{x}$ & $\mathrm{y}$ & $\mathrm{z}$ & $\mathrm{U}(\mathrm{eq})$ \\
\hline $\operatorname{Ag}(1)$ & $3350(1)$ & $4645(1)$ & $-37(1)$ & $31(1)$ \\
\hline $\operatorname{Ag}(2)$ & 2923(1) & $4847(1)$ & $1305(1)$ & $28(1)$ \\
\hline $\operatorname{Ag}(3)$ & 1818(1) & $6339(1)$ & $-287(1)$ & $25(1)$ \\
\hline $\mathrm{Rh}$ & 1901(1) & $3241(1)$ & $-39(1)$ & $16(1)$ \\
\hline $\mathrm{S}(1)$ & $3320(1)$ & 7494(1) & 744(1) & $31(1)$ \\
\hline$S(2)$ & $3155(1)$ & $3691(2)$ & $2780(1)$ & $33(1)$ \\
\hline $\mathrm{S}(3)$ & $415(1)$ & 8021(1) & $-997(1)$ & $24(1)$ \\
\hline $\mathrm{F}(1)$ & $3757(3)$ & $9559(5)$ & $440(3)$ & $87(2)$ \\
\hline $\mathrm{F}(2)$ & $3546(3)$ & $9617(5)$ & 1357(4) & $90(2)$ \\
\hline $\mathrm{F}(3)$ & $4417(2)$ & $8702(5)$ & 1357(3) & $76(2)$ \\
\hline $\mathrm{F}(4)$ & $3657(2)$ & $4966(4)$ & $3892(2)$ & $47(1)$ \\
\hline $\mathrm{F}(5)$ & 4381(2) & $4012(5)$ & $3622(2)$ & $59(1)$ \\
\hline$F(6)$ & $3786(2)$ & 2976(4) & 4018(2) & $50(1)$ \\
\hline $\mathrm{F}(7)$ & $98(3)$ & $8937(6)$ & $-2188(3)$ & $89(2)$ \\
\hline $\mathrm{F}(8)$ & $8(3)$ & $6902(6)$ & $-2152(2)$ & $88(2)$ \\
\hline $\mathrm{F}(9)$ & $-718(2)$ & $8127(7)$ & $-2025(2)$ & $83(2)$ \\
\hline $\mathrm{O}(1)$ & $3662(3)$ & $6800(6)$ & $399(4)$ & $76(2)$ \\
\hline $\mathrm{O}(2)$ & $3404(3)$ & $6880(5)$ & $1363(3)$ & $56(1)$ \\
\hline $\mathrm{O}(3)$ & $2675(3)$ & $7858(5)$ & 294(3) & $74(2)$ \\
\hline $\mathrm{O}(4)$ & $3242(3)$ & 4794(4) & $2434(2)$ & $46(1)$ \\
\hline $\mathrm{O}(5)$ & $3364(3)$ & $2538(5)$ & $2579(3)$ & $49(1)$ \\
\hline $\mathrm{O}(6)$ & $2536(2)$ & $3625(5)$ & $2844(2)$ & $45(1)$ \\
\hline $\mathrm{O}(7)$ & $1085(2)$ & $7855(4)$ & $-953(2)$ & $38(1)$ \\
\hline $\mathrm{O}(8)$ & $275(2)$ & $9218(4)$ & $-799(2)$ & $36(1)$ \\
\hline $\mathrm{O}(9)$ & $160(2)$ & 6981(4) & $-761(2)$ & $39(1)$ \\
\hline $\mathrm{N}(1)$ & 1960(2) & 1619(4) & $-574(2)$ & $23(1)$ \\
\hline $\mathrm{N}(2)$ & $1672(2)$ & 1909(4) & $565(2)$ & $21(1)$ \\
\hline $\mathrm{N}(3)$ & $870(2)$ & $3087(4)$ & $-649(2)$ & $22(1)$ \\
\hline $\mathrm{N}(4)$ & 4069(3) & $4823(6)$ & $-514(3)$ & $47(2)$ \\
\hline $\mathrm{C}(1)$ & 1889(3) & $496(5)$ & $-211(3)$ & $35(1)$ \\
\hline$C(2)$ & $2024(3)$ & $758(5)$ & $504(3)$ & $34(1)$ \\
\hline
\end{tabular}




\begin{tabular}{|c|c|c|c|c|}
\hline $\mathrm{C}(3)$ & $936(3)$ & $1715(6)$ & $280(3)$ & $33(1)$ \\
\hline $\mathrm{C}(4)$ & $569(3)$ & $2747(6)$ & $-170(3)$ & $34(1)$ \\
\hline $\mathrm{C}(5)$ & $767(3)$ & 2062(6) & $-1156(3)$ & $35(1)$ \\
\hline$C(6)$ & 1387(3) & $1747(6)$ & $-1238(3)$ & $36(1)$ \\
\hline $\mathrm{C}(7)$ & $2580(3)$ & 1511(6) & $-695(3)$ & $36(1)$ \\
\hline $\mathrm{C}(8)$ & 1908(3) & $2230(6)$ & $1284(3)$ & $33(1)$ \\
\hline $\mathrm{C}(9)$ & $546(3)$ & $4249(5)$ & $-1000(3)$ & $35(1)$ \\
\hline $\mathrm{C}(10)$ & 2872(3) & $3239(5)$ & $534(3)$ & $23(1)$ \\
\hline $\mathrm{C}(11)$ & $3475(3)$ & $3113(5)$ & $851(3)$ & $29(1)$ \\
\hline $\mathrm{C}(12)$ & $4165(3)$ & 2714(6) & 1204(3) & $37(1)$ \\
\hline $\mathrm{C}(13)$ & $4543(3)$ & $2445(7)$ & $821(4)$ & $45(2)$ \\
\hline $\mathrm{C}(14)$ & $5190(4)$ & $2006(8)$ & $1148(4)$ & $55(2)$ \\
\hline$C(15)$ & $5455(3)$ & 1841(7) & $1839(4)$ & $52(2)$ \\
\hline$C(16)$ & $5100(4)$ & $2100(9)$ & 2190(4) & $57(2)$ \\
\hline $\mathrm{C}(17)$ & $4434(4)$ & $2557(9)$ & $1866(4)$ & $58(2)$ \\
\hline $\mathrm{C}(18)$ & $1770(2)$ & $4714(5)$ & $463(2)$ & $19(1)$ \\
\hline $\mathrm{C}(19)$ & $1659(2)$ & $5579(5)$ & $779(3)$ & $21(1)$ \\
\hline $\mathrm{C}(20)$ & $1463(2)$ & $6443(5)$ & $1178(3)$ & $24(1)$ \\
\hline $\mathrm{C}(21)$ & $1427(3)$ & $6018(6)$ & 1771(3) & $31(1)$ \\
\hline $\mathrm{C}(22)$ & $1234(3)$ & $6825(7)$ & 2151(3) & $39(2)$ \\
\hline $\mathrm{C}(23)$ & 1064(3) & $8063(6)$ & 1942(3) & $36(2)$ \\
\hline $\mathrm{C}(24)$ & 1093(3) & $8479(6)$ & $1363(3)$ & $33(1)$ \\
\hline $\mathrm{C}(25)$ & 1297(3) & $7675(5)$ & $977(3)$ & $27(1)$ \\
\hline$C(26)$ & 2098(2) & $4387(5)$ & $-663(3)$ & $21(1)$ \\
\hline $\mathrm{C}(27)$ & 2251(3) & $5043(5)$ & $-1040(3)$ & $24(1)$ \\
\hline $\mathrm{C}(28)$ & 2443(3) & $5639(5)$ & $-1532(3)$ & $25(1)$ \\
\hline $\mathrm{C}(29)$ & 2541(3) & 4903(6) & $-2006(3)$ & $30(1)$ \\
\hline $\mathrm{C}(30)$ & $2766(3)$ & $5444(7)$ & $-2456(3)$ & $36(1)$ \\
\hline $\mathrm{C}(31)$ & 2886(3) & $6709(7)$ & $-2438(3)$ & $40(2)$ \\
\hline $\mathrm{C}(32)$ & 2787(3) & $7448(6)$ & $-1963(3)$ & $41(2)$ \\
\hline $\mathrm{C}(33)$ & 2571(3) & $6919(6)$ & $-1508(3)$ & $32(1)$ \\
\hline $\mathrm{C}(34)$ & $3797(4)$ & 8901(8) & $983(5)$ & $60(2)$ \\
\hline $\mathrm{C}(35)$ & $3775(3)$ & $3924(7)$ & $3621(3)$ & $39(2)$ \\
\hline$C(36)$ & $-70(4)$ & $7985(10)$ & $-1885(3)$ & $59(2)$ \\
\hline $\mathrm{C}(37)$ & 4303(3) & $4719(7)$ & $-893(4)$ & $42(2)$ \\
\hline $\mathrm{C}(38)$ & 4591(4) & $4556(8)$ & $-1383(4)$ & $57(2)$ \\
\hline
\end{tabular}


Table 3. Bond lengths $[\AA]$ and angles $\left[{ }^{\circ}\right]$ for 4 .

\begin{tabular}{|c|c|}
\hline $\operatorname{Ag}(1)-\mathrm{N}(4)$ & $2.220(6)$ \\
\hline $\operatorname{Ag}(1)-C(10)$ & $2.443(5)$ \\
\hline $\operatorname{Ag}(1)-C(11)$ & $2.468(6)$ \\
\hline $\operatorname{Ag}(1)-\mathrm{O}(1)$ & $2.482(7)$ \\
\hline $\operatorname{Ag}(1)-C(26)$ & $2.551(5)$ \\
\hline $\operatorname{Ag}(1)-C(27)$ & $2.565(5)$ \\
\hline $\mathrm{Ag}(2)-\mathrm{O}(4)$ & $2.279(4)$ \\
\hline $\mathrm{Ag}(2)-\mathrm{C}(10)$ & $2.378(5)$ \\
\hline $\operatorname{Ag}(2)-O(2)$ & $2.397(5)$ \\
\hline $\operatorname{Ag}(2)-C(18)$ & $2.467(5)$ \\
\hline $\operatorname{Ag}(2)-C(11)$ & $2.619(6)$ \\
\hline $\operatorname{Ag}(2)-C(19)$ & $2.662(5)$ \\
\hline $\operatorname{Ag}(3)-\mathrm{O}(7)$ & $2.337(4)$ \\
\hline $\operatorname{Ag}(3)-C(26)$ & $2.409(5)$ \\
\hline $\operatorname{Ag}(3)-C(18)$ & $2.418(5)$ \\
\hline $\mathrm{Ag}(3)-\mathrm{O}(3)$ & $2.424(5)$ \\
\hline $\operatorname{Ag}(3)-C(27)$ & $2.605(5)$ \\
\hline $\operatorname{Ag}(3)-C(19)$ & $2.617(5)$ \\
\hline $\mathrm{Rh}-\mathrm{C}(10)$ & $1.996(5)$ \\
\hline $\mathrm{Rh}-\mathrm{C}(26)$ & $2.004(5)$ \\
\hline Rh-C(18) & $2.004(5)$ \\
\hline $\mathrm{Rh}-\mathrm{N}(1)$ & $2.123(4)$ \\
\hline Rh-N(3) & $2.126(4)$ \\
\hline $\mathrm{Rh}-\mathrm{N}(2)$ & $2.133(4)$ \\
\hline $\mathrm{S}(1)-\mathrm{O}(3)$ & $1.417(5)$ \\
\hline $\mathrm{S}(1)-\mathrm{O}(2)$ & $1.447(6)$ \\
\hline $\mathrm{S}(1)-\mathrm{O}(1)$ & $1.462(6)$ \\
\hline$S(1)-C(34)$ & $1.788(8)$ \\
\hline $\mathrm{S}(2)-\mathrm{O}(6)$ & $1.423(5)$ \\
\hline $\mathrm{S}(2)-\mathrm{O}(5)$ & $1.443(5)$ \\
\hline $\mathrm{S}(2)-\mathrm{O}(4)$ & $1.455(5)$ \\
\hline$S(2)-C(35)$ & $1.813(7)$ \\
\hline $\mathrm{S}(3)-\mathrm{O}(8)$ & $1.423(4)$ \\
\hline $\mathrm{S}(3)-\mathrm{O}(9)$ & $1.430(4)$ \\
\hline
\end{tabular}

\begin{tabular}{|c|c|}
\hline $\mathrm{S}(3)-\mathrm{O}(7)$ & $1.446(4)$ \\
\hline $\mathrm{S}(3)-\mathrm{C}(36)$ & $1.802(7)$ \\
\hline $\mathrm{F}(1)-\mathrm{C}(34)$ & $1.352(10)$ \\
\hline $\mathrm{F}(2)-\mathrm{C}(34)$ & $1.385(11)$ \\
\hline $\mathrm{F}(3)-\mathrm{C}(34)$ & $1.294(9)$ \\
\hline $\mathrm{F}(4)-\mathrm{C}(35)$ & $1.332(8)$ \\
\hline $\mathrm{F}(5)-\mathrm{C}(35)$ & $1.334(7)$ \\
\hline $\mathrm{F}(6)-\mathrm{C}(35)$ & $1.326(8)$ \\
\hline $\mathrm{F}(7)-\mathrm{C}(36)$ & $1.344(11)$ \\
\hline $\mathrm{F}(8)-\mathrm{C}(36)$ & $1.338(11)$ \\
\hline $\mathrm{F}(9)-\mathrm{C}(36)$ & $1.339(9)$ \\
\hline $\mathrm{N}(1)-\mathrm{C}(1)$ & $1.480(7)$ \\
\hline $\mathrm{N}(1)-\mathrm{C}(7)$ & $1.493(7)$ \\
\hline $\mathrm{N}(1)-\mathrm{C}(6)$ & $1.497(7)$ \\
\hline $\mathrm{N}(2)-\mathrm{C}(8)$ & $1.485(7)$ \\
\hline $\mathrm{N}(2)-\mathrm{C}(2)$ & $1.486(7)$ \\
\hline $\mathrm{N}(2)-\mathrm{C}(3)$ & $1.497(7)$ \\
\hline $\mathrm{N}(3)-\mathrm{C}(9)$ & $1.484(7)$ \\
\hline $\mathrm{N}(3)-\mathrm{C}(4)$ & $1.491(7)$ \\
\hline $\mathrm{N}(3)-\mathrm{C}(5)$ & $1.508(7)$ \\
\hline $\mathrm{N}(4)-\mathrm{C}(37)$ & 1.143(9) \\
\hline$C(1)-C(2)$ & $1.495(9)$ \\
\hline $\mathrm{C}(1)-\mathrm{H}(1 \mathrm{~A})$ & 0.9900 \\
\hline $\mathrm{C}(1)-\mathrm{H}(1 \mathrm{~B})$ & 0.9900 \\
\hline $\mathrm{C}(2)-\mathrm{H}(2 \mathrm{~A})$ & 0.9900 \\
\hline $\mathrm{C}(2)-\mathrm{H}(2 \mathrm{~B})$ & 0.9900 \\
\hline $\mathrm{C}(3)-\mathrm{C}(4)$ & $1.481(9)$ \\
\hline $\mathrm{C}(3)-\mathrm{H}(3 \mathrm{~A})$ & 0.9900 \\
\hline $\mathrm{C}(3)-\mathrm{H}(3 \mathrm{~B})$ & 0.9900 \\
\hline $\mathrm{C}(4)-\mathrm{H}(4 \mathrm{~A})$ & 0.9900 \\
\hline $\mathrm{C}(4)-\mathrm{H}(4 \mathrm{~B})$ & 0.9900 \\
\hline$C(5)-C(6)$ & $1.483(9)$ \\
\hline $\mathrm{C}(5)-\mathrm{H}(5 \mathrm{~A})$ & 0.9900 \\
\hline $\mathrm{C}(5)-\mathrm{H}(5 \mathrm{~B})$ & 0.9900 \\
\hline $\mathrm{C}(6)-\mathrm{H}(6 \mathrm{~A})$ & 0.9900 \\
\hline $\mathrm{C}(6)-\mathrm{H}(6 \mathrm{~B})$ & 0.9900 \\
\hline
\end{tabular}




\begin{tabular}{|c|c|c|c|}
\hline $\mathrm{C}(7)-\mathrm{H}(7 \mathrm{~A})$ & 0.9800 & $C(27)-C(28)$ & $1.450(8)$ \\
\hline $\mathrm{C}(7)-\mathrm{H}(7 \mathrm{~B})$ & 0.9800 & $C(28)-C(29)$ & $1.385(8)$ \\
\hline $\mathrm{C}(7)-\mathrm{H}(7 \mathrm{C})$ & 0.9800 & $C(28)-C(33)$ & $1.393(8)$ \\
\hline $\mathrm{C}(8)-\mathrm{H}(8 \mathrm{~A})$ & 0.9800 & $C(29)-C(30)$ & $1.389(8)$ \\
\hline $\mathrm{C}(8)-\mathrm{H}(8 \mathrm{~B})$ & 0.9800 & $\mathrm{C}(29)-\mathrm{H}(29)$ & 0.9500 \\
\hline $\mathrm{C}(8)-\mathrm{H}(8 \mathrm{C})$ & 0.9800 & $\mathrm{C}(30)-\mathrm{C}(31)$ & $1.374(10)$ \\
\hline $\mathrm{C}(9)-\mathrm{H}(9 \mathrm{~A})$ & 0.9800 & $\mathrm{C}(30)-\mathrm{H}(30)$ & 0.9500 \\
\hline $\mathrm{C}(9)-\mathrm{H}(9 \mathrm{~B})$ & 0.9800 & $\mathrm{C}(31)-\mathrm{C}(32)$ & $1.390(10)$ \\
\hline $\mathrm{C}(9)-\mathrm{H}(9 \mathrm{C})$ & 0.9800 & $\mathrm{C}(31)-\mathrm{H}(31)$ & 0.9500 \\
\hline $\mathrm{C}(10)-\mathrm{C}(11)$ & $1.233(8)$ & $\mathrm{C}(32)-\mathrm{C}(33)$ & $1.382(9)$ \\
\hline $\mathrm{C}(11)-\mathrm{C}(12)$ & $1.462(8)$ & $\mathrm{C}(32)-\mathrm{H}(32)$ & 0.9500 \\
\hline $\mathrm{C}(12)-\mathrm{C}(17)$ & $1.336(10)$ & $\mathrm{C}(33)-\mathrm{H}(33)$ & 0.9500 \\
\hline $\mathrm{C}(12)-\mathrm{C}(13)$ & $1.423(10)$ & $\mathrm{C}(37)-\mathrm{C}(38)$ & $1.456(10)$ \\
\hline $\mathrm{C}(13)-\mathrm{C}(14)$ & $1.392(10)$ & $\mathrm{C}(38)-\mathrm{H}(38 \mathrm{~A})$ & 0.9800 \\
\hline $\mathrm{C}(13)-\mathrm{H}(13)$ & 0.9500 & $\mathrm{C}(38)-\mathrm{H}(38 \mathrm{~B})$ & 0.9800 \\
\hline $\mathrm{C}(14)-\mathrm{C}(15)$ & $1.393(11)$ & $\mathrm{C}(38)-\mathrm{H}(38 \mathrm{C})$ & 0.9800 \\
\hline $\mathrm{C}(14)-\mathrm{H}(14)$ & 0.9500 & & \\
\hline $\mathrm{C}(15)-\mathrm{C}(16)$ & $1.326(11)$ & $\mathrm{N}(4)-\operatorname{Ag}(1)-\mathrm{C}(10)$ & $145.5(2)$ \\
\hline $\mathrm{C}(15)-\mathrm{H}(15)$ & 0.9500 & $\mathrm{~N}(4)-\operatorname{Ag}(1)-\mathrm{C}(11)$ & $123.3(2)$ \\
\hline $\mathrm{C}(16)-\mathrm{C}(17)$ & $1.431(10)$ & $C(10)-A g(1)-C(11)$ & $29.09(18)$ \\
\hline $\mathrm{C}(16)-\mathrm{H}(16)$ & 0.9500 & $\mathrm{~N}(4)-\mathrm{Ag}(1)-\mathrm{O}(1)$ & $87.8(2)$ \\
\hline $\mathrm{C}(17)-\mathrm{H}(17)$ & 0.9500 & $\mathrm{C}(10)-\mathrm{Ag}(1)-\mathrm{O}(1)$ & $118.9(2)$ \\
\hline $\mathrm{C}(18)-\mathrm{C}(19)$ & $1.234(7)$ & $\mathrm{C}(11)-\mathrm{Ag}(1)-\mathrm{O}(1)$ & $112.7(2)$ \\
\hline $\mathrm{C}(19)-\mathrm{C}(20)$ & $1.449(7)$ & $\mathrm{N}(4)-\mathrm{Ag}(1)-\mathrm{C}(26)$ & $125.1(2)$ \\
\hline $\mathrm{C}(20)-\mathrm{C}(25)$ & $1.389(8)$ & $C(10)-\operatorname{Ag}(1)-C(26)$ & $67.32(17)$ \\
\hline $\mathrm{C}(20)-\mathrm{C}(21)$ & $1.404(8)$ & $C(11)-A g(1)-C(26)$ & $96.10(17)$ \\
\hline $\mathrm{C}(21)-\mathrm{C}(22)$ & $1.378(8)$ & $\mathrm{O}(1)-\mathrm{Ag}(1)-\mathrm{C}(26)$ & $112.3(2)$ \\
\hline $\mathrm{C}(21)-\mathrm{H}(21)$ & 0.9500 & $\mathrm{~N}(4)-\operatorname{Ag}(1)-\mathrm{C}(27)$ & $100.9(2)$ \\
\hline $\mathrm{C}(22)-\mathrm{C}(23)$ & $1.400(10)$ & $C(10)-A g(1)-C(27)$ & $95.00(17)$ \\
\hline $\mathrm{C}(22)-\mathrm{H}(22)$ & 0.9500 & $C(11)-\operatorname{Ag}(1)-C(27)$ & $123.48(18)$ \\
\hline $\mathrm{C}(23)-\mathrm{C}(24)$ & $1.366(9)$ & $\mathrm{O}(1)-\mathrm{Ag}(1)-\mathrm{C}(27)$ & $101.3(2)$ \\
\hline $\mathrm{C}(23)-\mathrm{H}(23)$ & 0.9500 & $C(26)-A g(1)-C(27)$ & $27.74(17)$ \\
\hline $\mathrm{C}(24)-\mathrm{C}(25)$ & $1.399(8)$ & $\mathrm{O}(4)-\mathrm{Ag}(2)-\mathrm{C}(10)$ & $130.96(18)$ \\
\hline $\mathrm{C}(24)-\mathrm{H}(24)$ & 0.9500 & $\mathrm{O}(4)-\mathrm{Ag}(2)-\mathrm{O}(2)$ & $91.55(18)$ \\
\hline $\mathrm{C}(25)-\mathrm{H}(25)$ & 0.9500 & $\mathrm{C}(10)-\mathrm{Ag}(2)-\mathrm{O}(2)$ & $125.94(19)$ \\
\hline $\mathrm{C}(26)-\mathrm{C}(27)$ & $1.227(8)$ & $\mathrm{O}(4)-\mathrm{Ag}(2)-\mathrm{C}(18)$ & $125.78(18)$ \\
\hline
\end{tabular}




\begin{tabular}{|c|c|c|c|}
\hline$C(10)-\operatorname{Ag}(2)-C(18)$ & $71.78(18)$ & $\mathrm{C}(10)-\mathrm{Rh}-\mathrm{N}(2)$ & 93.97(19) \\
\hline $\mathrm{O}(2)-\mathrm{Ag}(2)-\mathrm{C}(18)$ & $113.05(18)$ & $\mathrm{C}(26)-\mathrm{Rh}-\mathrm{N}(2)$ & $175.47(19)$ \\
\hline $\mathrm{O}(4)-\mathrm{Ag}(2)-\mathrm{C}(11)$ & $113.80(19)$ & $\mathrm{C}(18)-\mathrm{Rh}-\mathrm{N}(2)$ & $93.68(18)$ \\
\hline$C(10)-A g(2)-C(11)$ & $28.06(18)$ & $\mathrm{N}(1)-\mathrm{Rh}-\mathrm{N}(2)$ & $82.97(17)$ \\
\hline $\mathrm{O}(2)-\mathrm{Ag}(2)-\mathrm{C}(11)$ & $113.54(19)$ & $\mathrm{N}(3)-\mathrm{Rh}-\mathrm{N}(2)$ & $83.11(17)$ \\
\hline$C(18)-A g(2)-C(11)$ & $99.81(17)$ & $\mathrm{O}(3)-\mathrm{S}(1)-\mathrm{O}(2)$ & $119.4(4)$ \\
\hline $\mathrm{O}(4)-\mathrm{Ag}(2)-\mathrm{C}(19)$ & $106.92(18)$ & $\mathrm{O}(3)-\mathrm{S}(1)-\mathrm{O}(1)$ & 111.2(4) \\
\hline$C(10)-\operatorname{Ag}(2)-C(19)$ & $99.29(17)$ & $\mathrm{O}(2)-\mathrm{S}(1)-\mathrm{O}(1)$ & $110.6(4)$ \\
\hline $\mathrm{O}(2)-\mathrm{Ag}(2)-\mathrm{C}(19)$ & $96.82(18)$ & $\mathrm{O}(3)-\mathrm{S}(1)-\mathrm{C}(34)$ & $106.2(3)$ \\
\hline$C(18)-\operatorname{Ag}(2)-C(19)$ & $27.51(16)$ & $\mathrm{O}(2)-\mathrm{S}(1)-\mathrm{C}(34)$ & $105.5(4)$ \\
\hline$C(11)-\operatorname{Ag}(2)-C(19)$ & $127.31(17)$ & $\mathrm{O}(1)-\mathrm{S}(1)-\mathrm{C}(34)$ & $102.3(4)$ \\
\hline $\mathrm{O}(7)-\operatorname{Ag}(3)-\mathrm{C}(26)$ & $126.44(17)$ & $\mathrm{O}(6)-\mathrm{S}(2)-\mathrm{O}(5)$ & $114.9(3)$ \\
\hline $\mathrm{O}(7)-\mathrm{Ag}(3)-\mathrm{C}(18)$ & $135.49(16)$ & $\mathrm{O}(6)-\mathrm{S}(2)-\mathrm{O}(4)$ & $114.9(3)$ \\
\hline$C(26)-\operatorname{Ag}(3)-C(18)$ & $72.29(18)$ & $\mathrm{O}(5)-\mathrm{S}(2)-\mathrm{O}(4)$ & 114.1(3) \\
\hline $\mathrm{O}(7)-\mathrm{Ag}(3)-\mathrm{O}(3)$ & $93.43(17)$ & $\mathrm{O}(6)-\mathrm{S}(2)-\mathrm{C}(35)$ & $105.5(3)$ \\
\hline $\mathrm{C}(26)-\mathrm{Ag}(3)-\mathrm{O}(3)$ & $120.49(19)$ & $\mathrm{O}(5)-\mathrm{S}(2)-\mathrm{C}(35)$ & $103.0(3)$ \\
\hline $\mathrm{C}(18)-\mathrm{Ag}(3)-\mathrm{O}(3)$ & $111.17(19)$ & $\mathrm{O}(4)-\mathrm{S}(2)-\mathrm{C}(35)$ & $102.3(3)$ \\
\hline $\mathrm{O}(7)-\operatorname{Ag}(3)-\mathrm{C}(27)$ & 108.09(17) & $\mathrm{O}(8)-\mathrm{S}(3)-\mathrm{O}(9)$ & 115.1(3) \\
\hline$C(26)-A g(3)-C(27)$ & 27.97(17) & $\mathrm{O}(8)-\mathrm{S}(3)-\mathrm{O}(7)$ & $115.3(3)$ \\
\hline $\mathrm{C}(18)-\mathrm{Ag}(3)-\mathrm{C}(27)$ & 99.91(17) & $\mathrm{O}(9)-\mathrm{S}(3)-\mathrm{O}(7)$ & $114.6(3)$ \\
\hline $\mathrm{O}(3)-\mathrm{Ag}(3)-\mathrm{C}(27)$ & $106.5(2)$ & $\mathrm{O}(8)-\mathrm{S}(3)-\mathrm{C}(36)$ & $103.8(4)$ \\
\hline $\mathrm{O}(7)-\mathrm{Ag}(3)-\mathrm{C}(19)$ & $117.68(16)$ & $\mathrm{O}(9)-\mathrm{S}(3)-\mathrm{C}(36)$ & $102.9(4)$ \\
\hline$C(26)-\operatorname{Ag}(3)-C(19)$ & $100.24(17)$ & $\mathrm{O}(7)-\mathrm{S}(3)-\mathrm{C}(36)$ & $102.5(3)$ \\
\hline$C(18)-\operatorname{Ag}(3)-C(19)$ & $28.02(17)$ & $\mathrm{S}(1)-\mathrm{O}(1)-\mathrm{Ag}(1)$ & $123.2(4)$ \\
\hline $\mathrm{O}(3)-\operatorname{Ag}(3)-\mathrm{C}(19)$ & $95.4(2)$ & $\mathrm{S}(1)-\mathrm{O}(2)-\mathrm{Ag}(2)$ & $118.2(3)$ \\
\hline $\mathrm{C}(27)-\mathrm{Ag}(3)-\mathrm{C}(19)$ & $127.54(17)$ & $\mathrm{S}(1)-\mathrm{O}(3)-\mathrm{Ag}(3)$ & $122.0(3)$ \\
\hline $\mathrm{C}(10)-\mathrm{Rh}-\mathrm{C}(26)$ & $87.6(2)$ & $\mathrm{S}(2)-\mathrm{O}(4)-\mathrm{Ag}(2)$ & $122.4(3)$ \\
\hline $\mathrm{C}(10)-\mathrm{Rh}-\mathrm{C}(18)$ & $90.5(2)$ & $\mathrm{S}(3)-\mathrm{O}(7)-\mathrm{Ag}(3)$ & $123.8(3)$ \\
\hline $\mathrm{C}(26)-\mathrm{Rh}-\mathrm{C}(18)$ & $90.5(2)$ & $\mathrm{C}(1)-\mathrm{N}(1)-\mathrm{C}(7)$ & $109.4(5)$ \\
\hline $\mathrm{C}(10)-\mathrm{Rh}-\mathrm{N}(1)$ & $93.4(2)$ & $\mathrm{C}(1)-\mathrm{N}(1)-\mathrm{C}(6)$ & $111.4(5)$ \\
\hline $\mathrm{C}(26)-\mathrm{Rh}-\mathrm{N}(1)$ & 92.71(19) & $\mathrm{C}(7)-\mathrm{N}(1)-\mathrm{C}(6)$ & $108.0(5)$ \\
\hline $\mathrm{C}(18)-\mathrm{Rh}-\mathrm{N}(1)$ & $175.03(18)$ & $\mathrm{C}(1)-\mathrm{N}(1)-\mathrm{Rh}$ & $108.9(3)$ \\
\hline $\mathrm{C}(10)-\mathrm{Rh}-\mathrm{N}(3)$ & $175.51(19)$ & $\mathrm{C}(7)-\mathrm{N}(1)-\mathrm{Rh}$ & $114.9(3)$ \\
\hline $\mathrm{C}(26)-\mathrm{Rh}-\mathrm{N}(3)$ & $95.02(19)$ & $\mathrm{C}(6)-\mathrm{N}(1)-\mathrm{Rh}$ & $104.2(3)$ \\
\hline $\mathrm{C}(18)-\mathrm{Rh}-\mathrm{N}(3)$ & $93.08(18)$ & $\mathrm{C}(8)-\mathrm{N}(2)-\mathrm{C}(2)$ & $108.2(5)$ \\
\hline $\mathrm{N}(1)-\mathrm{Rh}-\mathrm{N}(3)$ & $82.91(17)$ & $\mathrm{C}(8)-\mathrm{N}(2)-\mathrm{C}(3)$ & $109.5(4)$ \\
\hline
\end{tabular}




\begin{tabular}{|c|c|c|c|}
\hline $\mathrm{C}(2)-\mathrm{N}(2)-\mathrm{C}(3)$ & $111.3(5)$ & $\mathrm{C}(6)-\mathrm{C}(5)-\mathrm{H}(5 \mathrm{~A})$ & 109.2 \\
\hline $\mathrm{C}(8)-\mathrm{N}(2)-\mathrm{Rh}$ & $115.6(3)$ & $\mathrm{N}(3)-\mathrm{C}(5)-\mathrm{H}(5 \mathrm{~A})$ & 109.2 \\
\hline $\mathrm{C}(2)-\mathrm{N}(2)-\mathrm{Rh}$ & $103.7(3)$ & $\mathrm{C}(6)-\mathrm{C}(5)-\mathrm{H}(5 \mathrm{~B})$ & 109.2 \\
\hline$C(3)-N(2)-R h$ & $108.5(3)$ & $\mathrm{N}(3)-\mathrm{C}(5)-\mathrm{H}(5 \mathrm{~B})$ & 109.2 \\
\hline $\mathrm{C}(9)-\mathrm{N}(3)-\mathrm{C}(4)$ & $108.1(5)$ & $\mathrm{H}(5 \mathrm{~A})-\mathrm{C}(5)-\mathrm{H}(5 \mathrm{~B})$ & 107.9 \\
\hline $\mathrm{C}(9)-\mathrm{N}(3)-\mathrm{C}(5)$ & 109.2(4) & $C(5)-C(6)-N(1)$ & $111.1(5)$ \\
\hline $\mathrm{C}(4)-\mathrm{N}(3)-\mathrm{C}(5)$ & $111.1(5)$ & $\mathrm{C}(5)-\mathrm{C}(6)-\mathrm{H}(6 \mathrm{~A})$ & 109.4 \\
\hline $\mathrm{C}(9)-\mathrm{N}(3)-\mathrm{Rh}$ & $115.5(3)$ & $\mathrm{N}(1)-\mathrm{C}(6)-\mathrm{H}(6 \mathrm{~A})$ & 109.4 \\
\hline $\mathrm{C}(4)-\mathrm{N}(3)-\mathrm{Rh}$ & $103.9(3)$ & $\mathrm{C}(5)-\mathrm{C}(6)-\mathrm{H}(6 \mathrm{~B})$ & 109.4 \\
\hline $\mathrm{C}(5)-\mathrm{N}(3)-\mathrm{Rh}$ & 109.0(3) & $\mathrm{N}(1)-\mathrm{C}(6)-\mathrm{H}(6 \mathrm{~B})$ & 109.4 \\
\hline $\mathrm{C}(37)-\mathrm{N}(4)-\mathrm{Ag}(1)$ & $160.6(6)$ & $\mathrm{H}(6 \mathrm{~A})-\mathrm{C}(6)-\mathrm{H}(6 \mathrm{~B})$ & 108.0 \\
\hline $\mathrm{N}(1)-\mathrm{C}(1)-\mathrm{C}(2)$ & $112.8(5)$ & $\mathrm{N}(1)-\mathrm{C}(7)-\mathrm{H}(7 \mathrm{~A})$ & 109.5 \\
\hline $\mathrm{N}(1)-\mathrm{C}(1)-\mathrm{H}(1 \mathrm{~A})$ & 109.0 & $\mathrm{~N}(1)-\mathrm{C}(7)-\mathrm{H}(7 \mathrm{~B})$ & 109.5 \\
\hline $\mathrm{C}(2)-\mathrm{C}(1)-\mathrm{H}(1 \mathrm{~A})$ & 109.0 & $\mathrm{H}(7 \mathrm{~A})-\mathrm{C}(7)-\mathrm{H}(7 \mathrm{~B})$ & 109.5 \\
\hline $\mathrm{N}(1)-\mathrm{C}(1)-\mathrm{H}(1 \mathrm{~B})$ & 109.0 & $\mathrm{~N}(1)-\mathrm{C}(7)-\mathrm{H}(7 \mathrm{C})$ & 109.5 \\
\hline $\mathrm{C}(2)-\mathrm{C}(1)-\mathrm{H}(1 \mathrm{~B})$ & 109.0 & $\mathrm{H}(7 \mathrm{~A})-\mathrm{C}(7)-\mathrm{H}(7 \mathrm{C})$ & 109.5 \\
\hline $\mathrm{H}(1 \mathrm{~A})-\mathrm{C}(1)-\mathrm{H}(1 \mathrm{~B})$ & 107.8 & $\mathrm{H}(7 \mathrm{~B})-\mathrm{C}(7)-\mathrm{H}(7 \mathrm{C})$ & 109.5 \\
\hline $\mathrm{N}(2)-\mathrm{C}(2)-\mathrm{C}(1)$ & $110.9(5)$ & $\mathrm{N}(2)-\mathrm{C}(8)-\mathrm{H}(8 \mathrm{~A})$ & 109.5 \\
\hline $\mathrm{N}(2)-\mathrm{C}(2)-\mathrm{H}(2 \mathrm{~A})$ & 109.5 & $\mathrm{~N}(2)-\mathrm{C}(8)-\mathrm{H}(8 \mathrm{~B})$ & 109.5 \\
\hline $\mathrm{C}(1)-\mathrm{C}(2)-\mathrm{H}(2 \mathrm{~A})$ & 109.5 & $\mathrm{H}(8 \mathrm{~A})-\mathrm{C}(8)-\mathrm{H}(8 \mathrm{~B})$ & 109.5 \\
\hline $\mathrm{N}(2)-\mathrm{C}(2)-\mathrm{H}(2 \mathrm{~B})$ & 109.5 & $\mathrm{~N}(2)-\mathrm{C}(8)-\mathrm{H}(8 \mathrm{C})$ & 109.5 \\
\hline $\mathrm{C}(1)-\mathrm{C}(2)-\mathrm{H}(2 \mathrm{~B})$ & 109.5 & $\mathrm{H}(8 \mathrm{~A})-\mathrm{C}(8)-\mathrm{H}(8 \mathrm{C})$ & 109.5 \\
\hline $\mathrm{H}(2 \mathrm{~A})-\mathrm{C}(2)-\mathrm{H}(2 \mathrm{~B})$ & 108.1 & $\mathrm{H}(8 \mathrm{~B})-\mathrm{C}(8)-\mathrm{H}(8 \mathrm{C})$ & 109.5 \\
\hline $\mathrm{C}(4)-\mathrm{C}(3)-\mathrm{N}(2)$ & $112.6(5)$ & $\mathrm{N}(3)-\mathrm{C}(9)-\mathrm{H}(9 \mathrm{~A})$ & 109.5 \\
\hline $\mathrm{C}(4)-\mathrm{C}(3)-\mathrm{H}(3 \mathrm{~A})$ & 109.1 & $\mathrm{~N}(3)-\mathrm{C}(9)-\mathrm{H}(9 \mathrm{~B})$ & 109.5 \\
\hline $\mathrm{N}(2)-\mathrm{C}(3)-\mathrm{H}(3 \mathrm{~A})$ & 109.1 & $\mathrm{H}(9 \mathrm{~A})-\mathrm{C}(9)-\mathrm{H}(9 \mathrm{~B})$ & 109.5 \\
\hline $\mathrm{C}(4)-\mathrm{C}(3)-\mathrm{H}(3 \mathrm{~B})$ & 109.1 & $\mathrm{~N}(3)-\mathrm{C}(9)-\mathrm{H}(9 \mathrm{C})$ & 109.5 \\
\hline $\mathrm{N}(2)-\mathrm{C}(3)-\mathrm{H}(3 \mathrm{~B})$ & 109.1 & $\mathrm{H}(9 \mathrm{~A})-\mathrm{C}(9)-\mathrm{H}(9 \mathrm{C})$ & 109.5 \\
\hline $\mathrm{H}(3 \mathrm{~A})-\mathrm{C}(3)-\mathrm{H}(3 \mathrm{~B})$ & 107.8 & $\mathrm{H}(9 \mathrm{~B})-\mathrm{C}(9)-\mathrm{H}(9 \mathrm{C})$ & 109.5 \\
\hline $\mathrm{C}(3)-\mathrm{C}(4)-\mathrm{N}(3)$ & $111.7(5)$ & $\mathrm{C}(11)-\mathrm{C}(10)-\mathrm{Rh}$ & $172.7(5)$ \\
\hline $\mathrm{C}(3)-\mathrm{C}(4)-\mathrm{H}(4 \mathrm{~A})$ & 109.3 & $\mathrm{C}(11)-\mathrm{C}(10)-\mathrm{Ag}(2)$ & $86.9(4)$ \\
\hline $\mathrm{N}(3)-\mathrm{C}(4)-\mathrm{H}(4 \mathrm{~A})$ & 109.3 & $\mathrm{Rh}-\mathrm{C}(10)-\operatorname{Ag}(2)$ & $100.3(2)$ \\
\hline $\mathrm{C}(3)-\mathrm{C}(4)-\mathrm{H}(4 \mathrm{~B})$ & 109.3 & $\mathrm{C}(11)-\mathrm{C}(10)-\mathrm{Ag}(1)$ & $76.6(4)$ \\
\hline $\mathrm{N}(3)-\mathrm{C}(4)-\mathrm{H}(4 \mathrm{~B})$ & 109.3 & $\mathrm{Rh}-\mathrm{C}(10)-\operatorname{Ag}(1)$ & $104.5(2)$ \\
\hline $\mathrm{H}(4 \mathrm{~A})-\mathrm{C}(4)-\mathrm{H}(4 \mathrm{~B})$ & 107.9 & $\operatorname{Ag}(2)-C(10)-A g(1)$ & $90.56(18)$ \\
\hline $\mathrm{C}(6)-\mathrm{C}(5)-\mathrm{N}(3)$ & $112.2(5)$ & $C(10)-C(11)-C(12)$ & $169.3(6)$ \\
\hline
\end{tabular}




\begin{tabular}{|c|c|c|c|}
\hline$C(10)-C(11)-A g(1)$ & $74.3(4)$ & $C(25)-C(20)-C(19)$ & $121.1(5)$ \\
\hline $\mathrm{C}(12)-\mathrm{C}(11)-\mathrm{Ag}(1)$ & $112.0(4)$ & $\mathrm{C}(21)-\mathrm{C}(20)-\mathrm{C}(19)$ & $119.3(5)$ \\
\hline $\mathrm{C}(10)-\mathrm{C}(11)-\mathrm{Ag}(2)$ & $65.0(4)$ & $\mathrm{C}(22)-\mathrm{C}(21)-\mathrm{C}(20)$ & $119.9(6)$ \\
\hline $\mathrm{C}(12)-\mathrm{C}(11)-\mathrm{Ag}(2)$ & 123.1(4) & $\mathrm{C}(22)-\mathrm{C}(21)-\mathrm{H}(21)$ & 120.0 \\
\hline $\operatorname{Ag}(1)-C(11)-\operatorname{Ag}(2)$ & $84.62(17)$ & $\mathrm{C}(20)-\mathrm{C}(21)-\mathrm{H}(21)$ & 120.0 \\
\hline $\mathrm{C}(17)-\mathrm{C}(12)-\mathrm{C}(13)$ & $120.0(6)$ & $\mathrm{C}(21)-\mathrm{C}(22)-\mathrm{C}(23)$ & $120.1(6)$ \\
\hline $\mathrm{C}(17)-\mathrm{C}(12)-\mathrm{C}(11)$ & $121.6(6)$ & $\mathrm{C}(21)-\mathrm{C}(22)-\mathrm{H}(22)$ & 120.0 \\
\hline $\mathrm{C}(13)-\mathrm{C}(12)-\mathrm{C}(11)$ & $118.3(6)$ & $\mathrm{C}(23)-\mathrm{C}(22)-\mathrm{H}(22)$ & 120.0 \\
\hline $\mathrm{C}(14)-\mathrm{C}(13)-\mathrm{C}(12)$ & $118.9(7)$ & $\mathrm{C}(24)-\mathrm{C}(23)-\mathrm{C}(22)$ & $120.2(6)$ \\
\hline $\mathrm{C}(14)-\mathrm{C}(13)-\mathrm{H}(13)$ & 120.6 & $\mathrm{C}(24)-\mathrm{C}(23)-\mathrm{H}(23)$ & 119.9 \\
\hline $\mathrm{C}(12)-\mathrm{C}(13)-\mathrm{H}(13)$ & 120.6 & $\mathrm{C}(22)-\mathrm{C}(23)-\mathrm{H}(23)$ & 119.9 \\
\hline $\mathrm{C}(13)-\mathrm{C}(14)-\mathrm{C}(15)$ & $119.8(7)$ & $\mathrm{C}(23)-\mathrm{C}(24)-\mathrm{C}(25)$ & $120.3(6)$ \\
\hline $\mathrm{C}(13)-\mathrm{C}(14)-\mathrm{H}(14)$ & 120.1 & $\mathrm{C}(23)-\mathrm{C}(24)-\mathrm{H}(24)$ & 119.8 \\
\hline $\mathrm{C}(15)-\mathrm{C}(14)-\mathrm{H}(14)$ & 120.1 & $\mathrm{C}(25)-\mathrm{C}(24)-\mathrm{H}(24)$ & 119.8 \\
\hline$C(16)-C(15)-C(14)$ & $120.8(7)$ & $\mathrm{C}(20)-\mathrm{C}(25)-\mathrm{C}(24)$ & $119.8(6)$ \\
\hline $\mathrm{C}(16)-\mathrm{C}(15)-\mathrm{H}(15)$ & 119.6 & $\mathrm{C}(20)-\mathrm{C}(25)-\mathrm{H}(25)$ & 120.1 \\
\hline $\mathrm{C}(14)-\mathrm{C}(15)-\mathrm{H}(15)$ & 119.6 & $\mathrm{C}(24)-\mathrm{C}(25)-\mathrm{H}(25)$ & 120.1 \\
\hline $\mathrm{C}(15)-\mathrm{C}(16)-\mathrm{C}(17)$ & $120.4(7)$ & $C(27)-C(26)-R h$ & $176.0(5)$ \\
\hline $\mathrm{C}(15)-\mathrm{C}(16)-\mathrm{H}(16)$ & 119.8 & $\mathrm{C}(27)-\mathrm{C}(26)-\mathrm{Ag}(3)$ & $84.9(4)$ \\
\hline $\mathrm{C}(17)-\mathrm{C}(16)-\mathrm{H}(16)$ & 119.8 & $\mathrm{Rh}-\mathrm{C}(26)-\mathrm{Ag}(3)$ & $98.2(2)$ \\
\hline $\mathrm{C}(12)-\mathrm{C}(17)-\mathrm{C}(16)$ & $120.1(7)$ & $C(27)-C(26)-A g(1)$ & $76.7(3)$ \\
\hline $\mathrm{C}(12)-\mathrm{C}(17)-\mathrm{H}(17)$ & 120.0 & $\mathrm{Rh}-\mathrm{C}(26)-\mathrm{Ag}(1)$ & $100.5(2)$ \\
\hline $\mathrm{C}(16)-\mathrm{C}(17)-\mathrm{H}(17)$ & 120.0 & $A g(3)-C(26)-A g(1)$ & $95.28(17)$ \\
\hline $\mathrm{C}(19)-\mathrm{C}(18)-\mathrm{Rh}$ & $176.0(4)$ & $\mathrm{C}(26)-\mathrm{C}(27)-\mathrm{C}(28)$ & $171.2(6)$ \\
\hline $\mathrm{C}(19)-\mathrm{C}(18)-\mathrm{Ag}(3)$ & $85.0(4)$ & $\mathrm{C}(26)-\mathrm{C}(27)-\mathrm{Ag}(1)$ & $75.5(3)$ \\
\hline $\mathrm{Rh}-\mathrm{C}(18)-\mathrm{Ag}(3)$ & $97.9(2)$ & $\mathrm{C}(28)-\mathrm{C}(27)-\mathrm{Ag}(1)$ & $104.6(3)$ \\
\hline $\mathrm{C}(19)-\mathrm{C}(18)-\operatorname{Ag}(2)$ & $85.1(3)$ & $\mathrm{C}(26)-\mathrm{C}(27)-\mathrm{Ag}(3)$ & $67.1(3)$ \\
\hline $\mathrm{Rh}-\mathrm{C}(18)-\mathrm{Ag}(2)$ & $97.13(19)$ & $\mathrm{C}(28)-\mathrm{C}(27)-\mathrm{Ag}(3)$ & $121.5(4)$ \\
\hline $\operatorname{Ag}(3)-C(18)-\operatorname{Ag}(2)$ & $98.81(17)$ & $\operatorname{Ag}(1)-C(27)-\operatorname{Ag}(3)$ & $90.36(17)$ \\
\hline $\mathrm{C}(18)-\mathrm{C}(19)-\mathrm{C}(20)$ & $170.4(5)$ & $\mathrm{C}(29)-\mathrm{C}(28)-\mathrm{C}(33)$ & $119.7(5)$ \\
\hline $\mathrm{C}(18)-\mathrm{C}(19)-\operatorname{Ag}(3)$ & $67.0(3)$ & $\mathrm{C}(29)-\mathrm{C}(28)-\mathrm{C}(27)$ & $119.0(5)$ \\
\hline $\mathrm{C}(20)-\mathrm{C}(19)-\operatorname{Ag}(3)$ & $121.0(4)$ & $\mathrm{C}(33)-\mathrm{C}(28)-\mathrm{C}(27)$ & $121.2(5)$ \\
\hline $\mathrm{C}(18)-\mathrm{C}(19)-\mathrm{Ag}(2)$ & $67.4(3)$ & $\mathrm{C}(28)-\mathrm{C}(29)-\mathrm{C}(30)$ & $119.9(6)$ \\
\hline $\mathrm{C}(20)-\mathrm{C}(19)-\operatorname{Ag}(2)$ & $116.0(3)$ & $\mathrm{C}(28)-\mathrm{C}(29)-\mathrm{H}(29)$ & 120.0 \\
\hline $\operatorname{Ag}(3)-C(19)-\operatorname{Ag}(2)$ & $89.30(15)$ & $\mathrm{C}(30)-\mathrm{C}(29)-\mathrm{H}(29)$ & 120.0 \\
\hline $\mathrm{C}(25)-\mathrm{C}(20)-\mathrm{C}(21)$ & $119.6(5)$ & $\mathrm{C}(31)-\mathrm{C}(30)-\mathrm{C}(29)$ & $120.6(6)$ \\
\hline
\end{tabular}




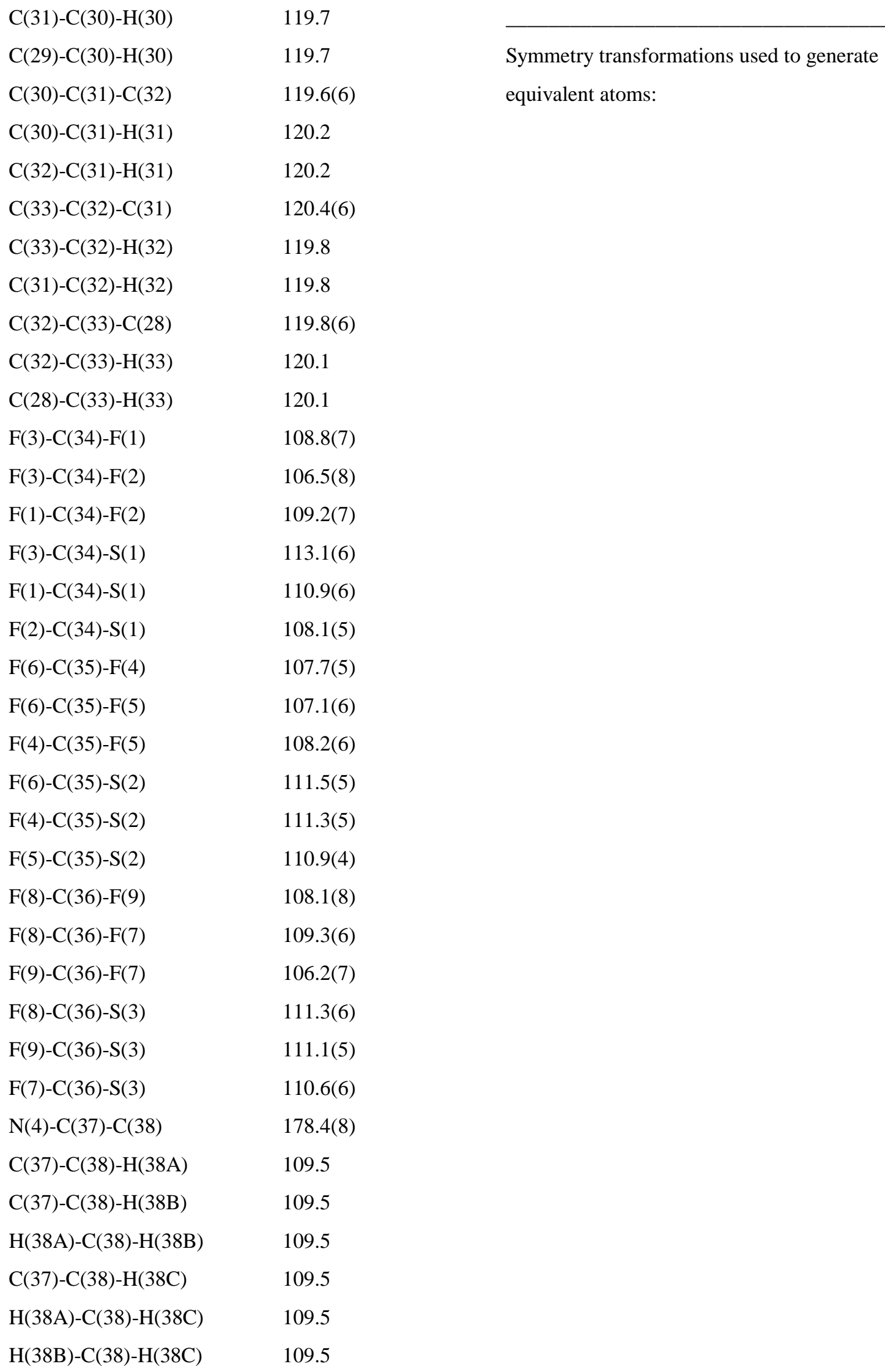


Table 4. Anisotropic displacement parameters $\left(\AA^{2} \times 10^{3}\right)$ for 4 . The anisotropic displacement factor exponent takes the form: $-2 \pi^{2}\left[h^{2} a^{* 2} U^{11}+\ldots+2 h k a^{*} b^{*} U^{12}\right]$

\begin{tabular}{|c|c|c|c|c|c|c|}
\hline & $\mathrm{U}^{11}$ & $\mathrm{U}^{22}$ & $\mathrm{U}^{33}$ & $\mathrm{U}^{23}$ & $\mathrm{U}^{13}$ & $\mathrm{U}^{12}$ \\
\hline $\operatorname{Ag}(1)$ & $21(1)$ & $36(1)$ & $36(1)$ & $6(1)$ & $11(1)$ & $-1(1)$ \\
\hline $\operatorname{Ag}(2)$ & $27(1)$ & $30(1)$ & $25(1)$ & $-1(1)$ & $6(1)$ & $-4(1)$ \\
\hline $\operatorname{Ag}(3)$ & $26(1)$ & $18(1)$ & $32(1)$ & $1(1)$ & $13(1)$ & $2(1)$ \\
\hline $\mathrm{Rh}$ & $13(1)$ & $15(1)$ & $20(1)$ & $0(1)$ & $8(1)$ & $0(1)$ \\
\hline$S(1)$ & $27(1)$ & $28(1)$ & $37(1)$ & $-1(1)$ & $11(1)$ & $-7(1)$ \\
\hline$S(2)$ & $37(1)$ & $38(1)$ & $27(1)$ & $2(1)$ & $15(1)$ & $-1(1)$ \\
\hline$S(3)$ & $23(1)$ & $20(1)$ & $29(1)$ & $0(1)$ & $10(1)$ & $2(1)$ \\
\hline $\mathrm{F}(1)$ & $89(4)$ & $54(3)$ & $92(4)$ & $30(3)$ & $9(3)$ & $-34(3)$ \\
\hline $\mathrm{F}(2)$ & $65(3)$ & $56(3)$ & $147(6)$ & $-34(3)$ & $42(4)$ & $-10(3)$ \\
\hline $\mathrm{F}(3)$ & $29(2)$ & $59(3)$ & $119(4)$ & $18(3)$ & $9(3)$ & $-10(2)$ \\
\hline $\mathrm{F}(4)$ & $48(2)$ & $56(3)$ & $36(2)$ & $-5(2)$ & $15(2)$ & $-6(2)$ \\
\hline $\mathrm{F}(5)$ & $29(2)$ & $89(4)$ & $61(3)$ & $6(3)$ & $19(2)$ & $-2(2)$ \\
\hline $\mathrm{F}(6)$ & $45(2)$ & $63(3)$ & $41(2)$ & $15(2)$ & $18(2)$ & $9(2)$ \\
\hline $\mathrm{F}(7)$ & $85(4)$ & $139(5)$ & $63(3)$ & $62(4)$ & $49(3)$ & $55(4)$ \\
\hline $\mathrm{F}(8)$ & $86(4)$ & $131(5)$ & $44(3)$ & $-42(3)$ & $24(3)$ & 11(4) \\
\hline $\mathrm{F}(9)$ & $32(2)$ & $160(6)$ & $45(3)$ & $-6(3)$ & $1(2)$ & $12(3)$ \\
\hline $\mathrm{O}(1)$ & $86(5)$ & $69(4)$ & $91(5)$ & $-12(4)$ & $56(4)$ & $-12(4)$ \\
\hline $\mathrm{O}(2)$ & $58(3)$ & $50(3)$ & $55(3)$ & $-2(3)$ & $16(3)$ & $-20(3)$ \\
\hline $\mathrm{O}(3)$ & $36(3)$ & $29(3)$ & $118(5)$ & $15(3)$ & $-11(3)$ & $-7(2)$ \\
\hline $\mathrm{O}(4)$ & $62(3)$ & $41(3)$ & $29(2)$ & $-2(2)$ & $13(2)$ & $-12(2)$ \\
\hline $\mathrm{O}(5)$ & $60(3)$ & $42(3)$ & $51(3)$ & $-4(2)$ & $30(3)$ & $-2(2)$ \\
\hline $\mathrm{O}(6)$ & $33(2)$ & $66(3)$ & $33(2)$ & $0(2)$ & $9(2)$ & $1(2)$ \\
\hline $\mathrm{O}(7)$ & $26(2)$ & $35(2)$ & $53(3)$ & $9(2)$ & $17(2)$ & $9(2)$ \\
\hline $\mathrm{O}(8)$ & $30(2)$ & $24(2)$ & $59(3)$ & $-9(2)$ & $22(2)$ & $-4(2)$ \\
\hline $\mathrm{O}(9)$ & $47(3)$ & $26(2)$ & $56(3)$ & $4(2)$ & $35(2)$ & $2(2)$ \\
\hline $\mathrm{N}(1)$ & $23(2)$ & $18(2)$ & $31(2)$ & $-3(2)$ & $14(2)$ & $-1(2)$ \\
\hline $\mathrm{N}(2)$ & $20(2)$ & $17(2)$ & $27(2)$ & $3(2)$ & $10(2)$ & $-1(2)$ \\
\hline $\mathrm{N}(3)$ & $16(2)$ & $19(2)$ & $27(2)$ & $-1(2)$ & $6(2)$ & $0(2)$ \\
\hline $\mathrm{N}(4)$ & $31(3)$ & $51(4)$ & $60(4)$ & $7(3)$ & $20(3)$ & $-1(3)$ \\
\hline $\mathrm{C}(1)$ & $48(4)$ & $16(3)$ & $47(4)$ & $3(2)$ & $26(3)$ & $1(2)$ \\
\hline
\end{tabular}




\begin{tabular}{|c|c|c|c|c|c|c|}
\hline$C(2)$ & $40(3)$ & $21(3)$ & $46(4)$ & $7(3)$ & $22(3)$ & $6(2)$ \\
\hline$C(3)$ & 21(3) & $37(3)$ & $44(3)$ & $4(3)$ & $16(3)$ & $-2(2)$ \\
\hline C(4) & 18(3) & $44(4)$ & $40(3)$ & $0(3)$ & $13(2)$ & $-1(2)$ \\
\hline$C(5)$ & 31(3) & $35(3)$ & $32(3)$ & $-10(3)$ & $5(3)$ & $0(3)$ \\
\hline$C(6)$ & $38(3)$ & $34(3)$ & $32(3)$ & $-8(3)$ & $10(3)$ & $-2(3)$ \\
\hline$C(7)$ & 41(3) & $30(3)$ & $51(4)$ & $-12(3)$ & $33(3)$ & $-1(3)$ \\
\hline $\mathrm{C}(8)$ & 44(3) & $32(3)$ & $25(3)$ & $3(2)$ & $17(3)$ & $-9(3)$ \\
\hline $\mathrm{C}(9)$ & $23(3)$ & $21(3)$ & $47(4)$ & $3(3)$ & 1(3) & $0(2)$ \\
\hline $\mathrm{C}(10)$ & $20(2)$ & $20(3)$ & $28(3)$ & $3(2)$ & $10(2)$ & $-2(2)$ \\
\hline $\mathrm{C}(11)$ & 26(3) & $23(3)$ & $37(3)$ & $6(2)$ & $10(2)$ & $0(2)$ \\
\hline $\mathrm{C}(12)$ & 21(3) & $32(3)$ & $49(4)$ & $6(3)$ & $5(3)$ & $-2(2)$ \\
\hline$C(13)$ & 41(4) & $46(4)$ & $45(4)$ & $-1(3)$ & $13(3)$ & $9(3)$ \\
\hline$C(14)$ & $38(4)$ & $62(5)$ & $67(5)$ & $3(4)$ & $23(4)$ & $18(4)$ \\
\hline$C(15)$ & $28(3)$ & $51(4)$ & $65(5)$ & $4(4)$ & $6(3)$ & $6(3)$ \\
\hline$C(16)$ & 43(4) & $84(6)$ & $39(4)$ & $16(4)$ & $10(3)$ & $19(4)$ \\
\hline$C(17)$ & $35(4)$ & $95(7)$ & $45(4)$ & $14(4)$ & $18(3)$ & $12(4)$ \\
\hline$C(18)$ & $13(2)$ & $24(3)$ & $19(2)$ & $4(2)$ & $4(2)$ & $-1(2)$ \\
\hline C(19) & $14(2)$ & $24(3)$ & $24(3)$ & $-1(2)$ & $6(2)$ & $-4(2)$ \\
\hline $\mathrm{C}(20)$ & $15(2)$ & $27(3)$ & $28(3)$ & $-8(2)$ & $7(2)$ & $-3(2)$ \\
\hline $\mathrm{C}(21)$ & $27(3)$ & $36(3)$ & $32(3)$ & $-5(3)$ & $14(2)$ & $-3(2)$ \\
\hline $\mathrm{C}(22)$ & $30(3)$ & $59(4)$ & $33(3)$ & $-15(3)$ & $17(3)$ & $-4(3)$ \\
\hline$C(23)$ & 23(3) & 41(4) & $45(4)$ & $-20(3)$ & $15(3)$ & $-1(3)$ \\
\hline$C(24)$ & 23(3) & $26(3)$ & $51(4)$ & $-13(3)$ & $14(3)$ & $-1(2)$ \\
\hline$C(25)$ & 20(3) & $25(3)$ & $33(3)$ & $-8(2)$ & $9(2)$ & $-2(2)$ \\
\hline$C(26)$ & $20(2)$ & $18(2)$ & $25(3)$ & $-1(2)$ & $10(2)$ & $-2(2)$ \\
\hline $\mathrm{C}(27)$ & 23(3) & $24(3)$ & $24(3)$ & $-1(2)$ & $9(2)$ & $2(2)$ \\
\hline$C(28)$ & 21(3) & $26(3)$ & $25(3)$ & $6(2)$ & $7(2)$ & $1(2)$ \\
\hline C(29) & $33(3)$ & $30(3)$ & $27(3)$ & $1(2)$ & $13(2)$ & $-2(2)$ \\
\hline $\mathrm{C}(30)$ & $32(3)$ & $52(4)$ & $24(3)$ & $5(3)$ & $13(2)$ & $-3(3)$ \\
\hline $\mathrm{C}(31)$ & 29(3) & $59(4)$ & $29(3)$ & $12(3)$ & $9(3)$ & $-10(3)$ \\
\hline $\mathrm{C}(32)$ & $37(3)$ & $35(4)$ & $47(4)$ & $13(3)$ & $14(3)$ & $-8(3)$ \\
\hline $\mathrm{C}(33)$ & $35(3)$ & $27(3)$ & $33(3)$ & $2(2)$ & $11(3)$ & $0(2)$ \\
\hline$C(34)$ & $38(4)$ & $41(4)$ & $93(7)$ & $11(4)$ & $17(4)$ & $-4(3)$ \\
\hline$C(35)$ & $33(3)$ & $53(4)$ & $31(3)$ & $2(3)$ & $14(3)$ & $-3(3)$ \\
\hline $\mathrm{C}(36)$ & 43(4) & $104(7)$ & $27(3)$ & $2(4)$ & $12(3)$ & $16(4)$ \\
\hline$C(37)$ & $36(4)$ & 41(4) & $55(4)$ & $4(3)$ & $23(3)$ & $-5(3)$ \\
\hline
\end{tabular}


C(38)

58(5)

53(5)

71(5)

$-6(4)$

39(4)

$-12(4)$ 
Table 5. Hydrogen coordinates ( $\left.\mathrm{x} 10^{4}\right)$ and isotropic displacement parameters $\left(\AA^{2} \mathrm{x} 10^{3}\right)$ for 4 .

\begin{tabular}{|c|c|c|c|c|}
\hline & $\mathrm{x}$ & $\mathrm{y}$ & $\mathrm{z}$ & $\mathrm{U}(\mathrm{eq})$ \\
\hline $\mathrm{H}(1 \mathrm{~A})$ & 2200 & -158 & -231 & 42 \\
\hline $\mathrm{H}(1 \mathrm{~B})$ & 1432 & 164 & -438 & 42 \\
\hline $\mathrm{H}(2 \mathrm{~A})$ & 1875 & 38 & 694 & 41 \\
\hline $\mathrm{H}(2 \mathrm{~B})$ & 2507 & 864 & 761 & 41 \\
\hline $\mathrm{H}(3 \mathrm{~A})$ & 829 & 918 & 28 & 39 \\
\hline $\mathrm{H}(3 \mathrm{~B})$ & 787 & 1646 & 650 & 39 \\
\hline $\mathrm{H}(4 \mathrm{~A})$ & 569 & 3490 & 101 & 40 \\
\hline $\mathrm{H}(4 \mathrm{~B})$ & 102 & 2489 & -422 & 40 \\
\hline $\mathrm{H}(5 \mathrm{~A})$ & 600 & 1304 & -1013 & 42 \\
\hline $\mathrm{H}(5 \mathrm{~B})$ & 426 & 2331 & -1591 & 42 \\
\hline $\mathrm{H}(6 \mathrm{~A})$ & 1486 & 2411 & -1500 & 43 \\
\hline $\mathrm{H}(6 \mathrm{~B})$ & 1326 & 951 & -1488 & 43 \\
\hline $\mathrm{H}(7 \mathrm{~A})$ & 2961 & 1401 & -268 & 54 \\
\hline $\mathrm{H}(7 \mathrm{~B})$ & 2641 & 2274 & -914 & 54 \\
\hline $\mathrm{H}(7 \mathrm{C})$ & 2546 & 788 & -983 & 54 \\
\hline $\mathrm{H}(8 \mathrm{~A})$ & 1808 & 1539 & 1524 & 49 \\
\hline $\mathrm{H}(8 \mathrm{~B})$ & 1684 & 2991 & 1335 & 49 \\
\hline $\mathrm{H}(8 \mathrm{C})$ & 2389 & 2372 & 1469 & 49 \\
\hline $\mathrm{H}(9 \mathrm{~A})$ & 68 & 4103 & -1241 & 52 \\
\hline $\mathrm{H}(9 \mathrm{~B})$ & 737 & 4491 & -1318 & 52 \\
\hline $\mathrm{H}(9 \mathrm{C})$ & 618 & 4922 & -673 & 52 \\
\hline $\mathrm{H}(13)$ & 4357 & 2565 & 351 & 55 \\
\hline $\mathrm{H}(14)$ & 5449 & 1818 & 901 & 66 \\
\hline $\mathrm{H}(15)$ & 5897 & 1541 & 2060 & 63 \\
\hline $\mathrm{H}(16)$ & 5287 & 1980 & 2661 & 69 \\
\hline $\mathrm{H}(17)$ & 4184 & 2749 & 2121 & 69 \\
\hline $\mathrm{H}(21)$ & 1537 & 5175 & 1910 & 37 \\
\hline $\mathrm{H}(22)$ & 1215 & 6541 & 2555 & 47 \\
\hline $\mathrm{H}(23)$ & 928 & 8615 & 2204 & 43 \\
\hline $\mathrm{H}(24)$ & 974 & 9318 & 1223 & 40 \\
\hline
\end{tabular}




\begin{tabular}{lllll}
$\mathrm{H}(25)$ & 1322 & 7970 & 577 & 32 \\
$\mathrm{H}(29)$ & 2455 & 4030 & -2025 & 36 \\
$\mathrm{H}(30)$ & 2836 & 4936 & -2778 & 43 \\
$\mathrm{H}(31)$ & 3037 & 7075 & -2749 & 48 \\
$\mathrm{H}(32)$ & 2868 & 8323 & -1950 & 49 \\
$\mathrm{H}(33)$ & 2509 & 7427 & -1180 & 39 \\
$\mathrm{H}(38 \mathrm{~A})$ & 5022 & 4138 & -1173 & 85 \\
$\mathrm{H}(38 \mathrm{~B})$ & 4651 & 5376 & -1553 & 85 \\
$\mathrm{H}(38 \mathrm{C})$ & 4294 & 4043 & -1755 & 85 \\
& & & & \\
\hline
\end{tabular}

